\title{
Learned Global Optimization for Inverse Scattering Problems - Matching Global Search with Computational Efficiency
}

M. Salucci, ${ }^{(1)}$ Member, IEEE, L. Poli, ${ }^{(1)}$ Member, IEEE, P. Rocca, ${ }^{(1)(2)}$ Senior Member, IEEE, and A. Massa, ${ }^{(1)(3)(4)}$ Fellow, IEEE

(1) CNIT - "University of Trento" Research Unit

Via Sommarive 9, 38123 Trento - Italy

E-mail: \{marco.salucci, lorenzo.poli, paolo.rocca, andrea.massa $@$ unitn.it

Website: www.eledia.org/eledia-unitn

(2)ELEDIA Research Center (ELEDIA@XIDIAN - Xidian University)

P.O. Box 191, No.2 South Tabai Road, 710071 Xi'an, Shaanxi Province - China

E-mail: paolo.rocca@xidian.edu.cn

Website: www.eledia.org/eledia-xidian

(3) ELEDIA Research Center (ELEDIA@UESTC - UESTC)

School of Electronic Engineering, Chengdu 611731 - China

E-mail: andrea.massa@uestc.edu.cn

Website: www.eledia.org/eledia-uestc

(4)ELEDIA Research Center (ELEDIA@TSINGHUA - Tsinghua University)

30 Shuangqing Rd, 100084 Haidian, Beijing - China

E-mail: andrea.massa@tsinghua.edu.cn

Website: www.eledia.org/eledia-tsinghua 


\title{
Learned Global Optimization for Inverse Scattering Problems \\ - Matching Global Search with Computational Efficiency
}

M. Salucci, L. Poli, P. Rocca, and A. Massa

\begin{abstract}
The computationally-efficient solution of fully non-linear microwave inverse scattering problems $(I S P \mathrm{~s})$ is addressed. An innovative System-by-Design $(S b D)$ based method is proposed to enable, for the first time to the best of the authors' knowledge, an effective, robust, and time-efficient exploitation of an evolutionary algorithm $(E A)$ to perform the global minimization of the data-mismatch cost function. According to the $S b D$ paradigm as suitably applied to $I S P \mathrm{~s}$, the proposed approach founds on (i) a smart re-formulation of the ISP based on the definition of a minimum-dimensionality and representative set of degrees-of-freedom (DoFs) and on (ii) the artificial-intelligence (AI)-driven integration of a customized global search technique with a digital twin $(D T)$ predictor based on the Gaussian Process $(G P)$ theory. Representative numerical and experimental results are provided to assess the effectiveness and the efficiency of the proposed approach also in comparison with competitive state-of-the-art inversion techniques.
\end{abstract}

Key words: Inverse Scattering $(I S)$, Evolutionary Algorithms (EAs), System-by-Design (SbD), Digital Twin $(D T)$, Artificial Intelligence $(A I)$, Learning-by-Examples ( $L B E)$, Gaussian Processes $(G P \mathrm{~s})$ 


\section{Introduction}

In microwave imaging, an electromagnetic $(E M)$ source illuminates an inaccessible investigation domain to be non-invasively reconstructed by inverting the scattered field data collected in an external observation domain [1]. Depending on the application at hand, both qualitative (i.e., detection, localization, and shaping) and quantitative (i.e., EM properties characterization) reconstructions can be yielded by solving an inverse scattering problem (ISP). ISPs arise in free-space imaging, biomedical diagnostics [2]-[6], subsurface and ground penetrating radar $(G P R)$ investigations [7]-[10], non-destructive testing and evaluation $(N D T / N D E)$ [11]-[14], and through-the-wall imaging (TWI) [15]-[17]. Recently, microwave imaging techniques, based on inverse scattering $(I S)$ formulations, have been also successfully applied to innovative contexts such as, for instance, food quality assessment [18]-[20]. However, solving an ISP is not a trivial task and it poses several challenges due to the intrinsic complexity of the scattering phenomena in the microwave regime described by the Maxwell's equations. First, the non-uniqueness of the solution, caused by the presence of non-radiating currents induced in the investigation domain, that do not contribute to the scattered data. Second, the non-linearity related to the multiple scattering effects [1]. To properly address such issues for yielding robust/reliable data-inversions, many effective strategies have appeared in the state-of-the-art literature. For instance, Born-based [21] and Rytov-based [22] approximations simplify the $I S$ equations as linearly depending on the unknown contrast distribution. However, they have limited applications to weak scatterers. Otherwise, innovative reformulations of the scattering equations as, for instance, the contraction integral equation $(C I E)$ method, have been introduced to deal with the non-linearity by properly redefining the contrast function [15][23]. Differently, contrast source inversion $(C S I)$ techniques proved to be an effective alternative to the linearization of the data equation [24], even though they are subject to the non-uniqueness of the arising inverse source problem so that multiplicative regularizations have been investigated [25][26].

Regardless of the formulation and unless closed-form solutions, ISPs are generally solved with deterministic $(D O)$ or global $(G O)$ optimization techniques. Strategies belonging to the former class include the subspace optimization method (SOM) [27]-[29], the conjugate gradient $(C G)$ [30], and the inexact Newton method (INM) [31]. To deterministically explore the solution 
space, these methods typically require the analytic/numerical differentiation of the cost function to be minimized. Consequently, they exhibit a high computational efficiency, but they can be trapped into local-minima/false-solutions, unless properly initialized within the so-called "attraction basin" of the global optimum.

As for $G O$ methods, nature-inspired strategies (i.e., evolutionary algorithms (EAs) [32]-[34]) such as genetic algorithms (GAs) [14], particle swarm optimization (PSO) [10], and differential evolution $(D E)$ [35] have been successfully applied to solve ISPs. Thanks to the "hill-climbing" features, they perform an effective global exploration of the solution space by evolving a population of trial solutions with stochastic operators [32] to "escape" from local minima, while converging towards the global optimum. Although successful in several ISP applications and more effective than $D O$ s in sampling nonlinear cost functions, EA-GOs are inherently limited by the computational burden. Indeed, the $C P U$ cost of a stochastic $G O$ is directly linked to the number of agents that evolve throughout the optimization process, which is in turn proportional to the number of degrees-of-freedom ( $D o F \mathrm{~s})$ that define the dimensionality of the solution space. To partially counteract such a limitation, one practical and effective solution is the integration of $E A-G O$ s with multi-resolution $(M R)$ strategies such as the iterative multi-scaling approach (IMSA) [36]. By adaptively refining the spatial resolution of the reconstruction only within the so-called regions-of-interest $(R o I)$, where the unknown scatterer has been detected, the number of unknowns is strongly reduced at each $M R$ step [10][37] by making computationally-feasible an $E A-G O$-based optimization.

On the other hand, artificial intelligence $(A I)$-based techniques, belonging to the so-called deep learning $(D L)$ framework [38]-[41], have shown an unprecedented computational efficiency in addressing the pixel-wise inversion of scattered data. However, they still present some unsolved challenges such as the need of huge amounts of training datasets to calibrate thousands of hyperparameters that define the underlying complex neural network $(N N)$ architecture composed by several hidden layers [38]. Within the $A I$ context, the System-by-Design $(S b D)$ has rapidly emerged as an innovative paradigm for the optimization-driven solution of complex EM problems [42]. The problem at hand is first decomposed into a set of sub-tasks implemented into suitably-defined functional blocks jointly designed with the shared goal of an effective, reliable, 
and computationally-efficient exploitation of $G O$ s. Such a goal is attained by $(i)$ re-formulating the problem at hand as a $G O$ one described by a minimum-dimensionality set of $D o F$ s and (ii) integrating EA-based strategies with fast analysis tools or digital twins (DTs), generated with learning-by-examples ( $L B E$ s) techniques [43], to speed up the evaluation (i.e., the cost function computation) of each trial solution. Thanks to its effectiveness and efficiency, the $S b D$ has been already successfully applied to many $E M$ design problems including the synthesis of single radiators [44], wide angle impedance matching layers [45], reflectarrays [46], and meta-material devices [47], but not to ISPs. This paper is then aimed at assessing the $S b D$ in reliably solving fully non-linear ISPs with a computational efficiency, comparable to that of DOs, towards the "holy-grail" of a global real-time optimization.

The paper is organized as follows. The ISP is described and mathematically formulated in Sect. II. Section III details the customization of the $S b D$ paradigm to ISPs and its implementation. Numerical and experimental results are shown in Sect. IV to prove the effectiveness and the efficiency of the proposed method in different operative conditions. Eventually, some conclusions and final remarks are drawn (Sect. V).

\section{Mathematical Formulation}

Without loss of generality, let us consider a two-dimensional $(2 D)$ scenario comprising a square investigation domain $D$ located within a homogeneous, lossless (i.e., conductivity $\sigma=\sigma_{0}=$ $0[\mathrm{~S} / \mathrm{m}]$ ), and non-magnetic (i.e., permeability $\mu=\mu_{0}$ ) background medium of permittivity $\varepsilon_{0}$. By assuming a time-harmonic dependence $\exp (-j 2 \pi f t), f$ being the working frequency, and a transverse magnetic (TM) (i.e., z-oriented) polarization of the $E M$ field, the scattering phenomena excited by a set of $V$ monochromatic incident fields, $\left\{\mathcal{I}^{(v)}(x, y) ; v=1, \ldots, V\right\}$, which illuminate the investigation domain $D$, in any $(x, y) \in D$ are modeled by the following State Equation [1]

$$
\mathcal{I}^{(v)}(x, y)=\mathcal{T}^{(v)}(x, y)-\int_{D} \mathcal{G}\left(x, y, x^{\prime}, y^{\prime}\right) \mathcal{J}^{(v)}\left(x^{\prime}, y^{\prime}\right) d x^{\prime} d y^{\prime}
$$

where 


$$
\mathcal{J}^{(v)}(x, y)=\tau(x, y) \mathcal{T}^{(v)}(x, y)
$$

is the $v$-th $(v=1, \ldots, V)$ equivalent current induced within $D, \mathcal{T}^{(v)}(x, y)$ is the total field, and

$$
\tau(x, y)=\left[\varepsilon_{r}(x, y)-1\right]+j \frac{\sigma(x, y)}{2 \pi f \varepsilon_{0}}
$$

is the contrast function that mathematically models the presence, within $D$, of an unknown scatterer with support $\Omega$ (i.e., $\tau(x, y) \neq 0$ when $(x, y) \in \Omega$ ) whose relative permittivity and conductivity distributions are equal to $\varepsilon_{r}(x, y)\left[\varepsilon_{r}(x, y) \triangleq \frac{\varepsilon(x, y)}{\varepsilon_{0}}\right]$ and $\sigma(x, y)$, respectively. Moreover,

$$
\mathcal{G}\left(x, y, x^{\prime}, y^{\prime}\right)=j \frac{k_{0}^{2}}{4} \mathcal{H}_{0}^{(1)}\left(k_{0} \sqrt{\left(x-x^{\prime}\right)^{2}+\left(y-y^{\prime}\right)^{2}}\right)
$$

is the $2 D$ Green's function of the background medium, $\mathcal{H}_{0}^{(1)}$ being the zero-th order Hankel's function of the first kind, and $k_{0}$ is the wavenumber $\left(k_{0} \triangleq 2 \pi f \sqrt{\varepsilon_{0} \mu_{0}}\right)$.

Otherwise, the $E M$ interactions in the external observation domain $O \notin D(O \cap D=\{0\})[1]$ are described by the Data Equation

$$
\mathcal{S}^{(v)}(x, y)=\int_{D} \mathcal{G}\left(x, y, x^{\prime}, y^{\prime}\right) \mathcal{J}^{(v)}\left(x^{\prime}, y^{\prime}\right) d x^{\prime} d y^{\prime}
$$

where $\mathcal{S}^{(v)}(x, y)\left[\mathcal{S}^{(v)}(x, y) \triangleq \mathcal{T}^{(v)}(x, y)-\mathcal{I}^{(v)}(x, y)\right]$ is the scattered field radiated in freespace by the $v$-th $(v=1, \ldots, V)$ equivalent source, $\mathcal{J}^{(v)}(x, y)$, and embedding the information on the unknown scatterer distribution in $D$.

To numerically deal with (5), the method-of-moments $(M o M)$ is applied by partitioning $D$ into $N$ square sub-domains, $D_{n}$ being the $n$-th $(n=1, \ldots, N)$ discretization domain $\left(D=\sum_{n=1}^{N} D_{n}\right)$ centered at $\left(x_{n}, y_{n}\right)$ and using $M$ Dirac's test functions to sample the scattered field at $M$ locations in $O, \underline{\mathcal{S}}^{(v)}=\left\{\mathcal{S}^{(v)}\left(x_{m}, y_{m}\right) ; m=1, \ldots, M\right\}$. The discrete form of (5) is then derived

$$
\underline{\mathcal{S}}^{(v)}=\underline{\underline{G}}_{O} \underline{\mathcal{J}}^{(v)}
$$

where $\underline{\mathcal{J}}^{(v)}=\left\{\mathcal{J}^{(v)}\left(x_{n}, y_{n}\right) ; n=1, \ldots, N\right\}$ and $\underline{\underline{G}}_{O}$ is the $(M \times N)$ external Green's matrix 
whose $(m, n)$-th $(m=1, \ldots, M ; n=1, \ldots, N)$ entry is given by $\left.\underline{\underline{G}}_{O}\right\rfloor_{m n}=j \frac{k_{0}^{2}}{4} \int_{D_{n}} \mathcal{H}_{0}^{(1)}\left(k_{0} \rho_{m}\right)$ $d x^{\prime} d y^{\prime}$ being $\rho_{m} \triangleq \sqrt{\left(x_{m}-x^{\prime}\right)^{2}+\left(y_{m}-y^{\prime}\right)^{2}}$.

Accordingly, the inverse problem at hand can be stated as follows

$\boldsymbol{I S P}$ - Starting from the knowledge of the incident, $\left\{\mathcal{I}^{(v)}\left(x_{n}, y_{n}\right) ; n=1, \ldots, N\right\}$, and the scattered, $\left\{\mathcal{S}^{(v)}\left(x_{m}, y_{m}\right) ; m=1, \ldots, M\right\}$, data samples, determine the contrast function distribution, $\left\{\tau\left(x_{n}, y_{n}\right) ; n=1, \ldots, N\right\}$, by solving (6).

In order to solve this full non-linear ISP, an innovative $S b D$-based is adopted according to the implementation detailed in Sect. 3.

\section{$3 \quad S b D$-Based Inversion Method}

According to the $S b D$ paradigm, the solution of the ISP relies on the exploitation of four interconnected functional blocks, each performing a specific sub-task (Fig. 1). The design and implementation of each block is strongly correlated to the other ones and it is driven by the following shared goals [42]: $(i)$ to yield an effective and reliable solution of the fully non-linear $I S P$. From an optimization viewpoint, it means to guarantee the convergence towards the global optimum; (ii) to reduce the computational burden required by a standard non-deterministic exploration of the solution space. In other words, the proposed $S b D$ approach is aimed at overcoming the limitation of $D O$ s, which cannot avoid being trapped into local minima unless properly initialized in the "attraction basin" of the actual-solution/global-optimum, while yielding competitive computational performance in solving the ISP so that the following condition on the required $C P U$-time holds true

$$
\Delta t_{S b D} \simeq \Delta t_{D O} \ll \Delta t_{G O}
$$

More specifically, the $S b D$ as applied to ISPs is implemented by defining the following blocks (Fig. 1):

1. Problem Formulation $(P F)$ - This block reformulates the ISP to enable an effective, reliable, and computationally-efficient exploitation of $G O$ s by coding the ISP unknowns 
into a minimum-dimension (yet highly-flexible) set of $K$ degrees-of-freedom ( $D o F$ s), $\underline{\xi}=\left\{\xi_{k} ; k=1, \ldots, K\right\}$, to give a "smart" representation of the solution space. Moreover, it defines a suitable cost function, $\Phi(\underline{\xi})$, which quantifies the quality of the solution in terms of data mismatch and it represents the unique link between the computational world and the physical one;

2. Data Computation $(D C)$ - In this block, the set of $S b D-D o F \mathrm{~s}, \underline{\xi}$, is mapped into a pixelbased representation of the equivalent currents induced within $D,\left\{\underline{\mathcal{J}}^{(v)} ; v=1, \ldots, V\right\}$, by means of (2) and (1) to compute, through (6), the scattered field distribution in $O$;

3. Cost Function Evaluation (CFE) - This block efficiently evaluates the cost function with a computationally-fast digital twin (DT) [43] of the accurate, but time-consuming, fullwave solver. It is the "engine" of the $S b D$-based inversion and it exploits the $D C$ block for the computation of the scattered data, $\underline{\widetilde{\mathcal{S}}}^{(v)}$, in correspondence with each coded trial solution, $\underline{\xi}$;

4. Solution Space Exploration (SSE) - This block performs an effective sampling of the ISP solution space by leveraging on (a) the "hill-climbing" features of a properly customized $E A$ strategy and on $(b)$ the smart interaction with the $D T$ to yield a fast and reliable convergence towards the global optimum. The SSE block receives as external inputs the samples of the incident, $\left\{\mathcal{I}^{(v)}\left(x_{n}, y_{n}\right),\left(x_{n}, y_{n}\right) \in D ; n=1, \ldots, N\right\}$, and the scattered, $\left\{\mathcal{S}^{(v)}\left(x_{m}, y_{m}\right),\left(x_{m}, y_{m}\right) \in O ; m=1, \ldots, M\right\}$, fields, while it uses the unknowns coding, $\underline{\xi}$, and the cost function definition, $\Phi$, from the $P F$ block. The $S S E$ output is the $S b D$ solution, $\underline{\xi}^{(S b D)}$, and its mapping in a contrast distribution, $\underline{\tau}^{(S b D)}$.

Each $S b D$ block is detailed in the following by pointing out the key-item for its integrated implementation.

\subsection{Problem Formulation $(P F)$}

Concerning the identification of a suitable parametric model of the ISP solution in terms of a limited set of $K$ descriptors, $\underline{\xi}=\left\{\xi_{k} ; k=1, \ldots, K\right\}$, it is worth noticing that the number of $D o F \mathrm{~s} K$ is directly proportional to the size of the population of trial-solutions, $P$, used in the 
multiple-agent minimization of $\Phi(\underline{\xi})$, and it determines the overall computational cost of the inversion process. Therefore, it is paramount to seek for the smartest coding of the solution that minimizes the computational burden of the optimization, while enabling a careful exploration of the solution space towards the global optimum $\underline{\xi}^{(o p t)}\left(\Phi\left(\underline{\xi}^{(o p t)}\right) \triangleq 0\right)$. Moreover, one should consider that the definition of a minimum-dimensionality representation of the ISP solution facilitates the generation of an accurate surrogate model (i.e., the $D T$ ) able to predict $\Phi(\underline{\xi})$ from a reduced set of training observations (see Sect. 3.3). Following this line of reasoning, a standard pixel-based representation of the unknown distribution of the $E M$ profile of $D, \underline{\tau}=$ $\left\{\Re\left(\tau_{n}\right), \Im\left(\tau_{n}\right) ; n=1, \ldots, N\right\}, \Re(.) / \Im($.$) being the real/imaginary part and \tau_{n}=\tau\left(x_{n}, y_{n}\right)$ $(n=1, \ldots, N)$, is sub-optimal because of the huge dimension of the corresponding solution space (i.e., $K=2 \times N$ ) [10]. To reduce the cardinality of the problem at hand, spline basis functions [47] are exploited here to model the external contour $\partial \Omega(x, y)$ of the homogeneous (1) scatterer (i.e., $\tau(x, y)=\tau_{\Omega},(x, y) \in \Omega$ ) of extension/support $\Omega$ (Fig. 2). More in detail, the 2-D profile $\partial \Omega(x, y)$ is expanded into $Q$ quadratic Bezier spline functions

$$
\partial \Omega(x, y)=\sum_{q=1}^{Q} \mathcal{B}^{(q)}(\alpha)
$$

the $q$-th basis function $(q=1, \ldots, Q)$ being given by

$$
\mathcal{B}^{(q)}(\alpha)=(1-\alpha)^{2}\left[\begin{array}{l}
\mathcal{V}_{x}^{(q)} \\
\mathcal{V}_{y}^{(q)}
\end{array}\right]+2 \alpha(1-\alpha)\left[\begin{array}{l}
\mathcal{C}_{x}^{(q)} \\
\mathcal{C}_{y}^{(q)}
\end{array}\right]+\alpha^{2}\left[\begin{array}{l}
\mathcal{V}_{x}^{(q+1)} \\
\mathcal{V}_{y}^{(q+1)}
\end{array}\right]
$$

where $\alpha \in[0,1]$ and $\mathcal{C}^{(q)}=\left(\mathcal{C}_{x}^{(q)}, \mathcal{C}_{y}^{(q)}\right)$ is the $q$-th $(q=1, \ldots, Q)$ control point of the spline profile whose coordinates are (Fig. 2)

$$
\left\{\begin{array}{l}
\mathcal{C}_{x}^{(q)}=x_{\Omega}+\rho^{(q)} \times \cos \left((q-1) \frac{2 \pi}{Q}\right) \\
\mathcal{C}_{y}^{(q)}=y_{\Omega}+\rho^{(q)} \times \sin \left((q-1) \frac{2 \pi}{Q}\right)
\end{array},\right.
$$

\footnotetext{
${ }^{(1)}$ The extension of the spline representation to doubly-connected contours (e.g., inhomogeneous concentric contrast distributions) as well as to multiple disconnected objects is straightforward as discussed and proved in Sect. 4
} 
while $\rho^{(q)}\left(\rho^{(q)}>0\right)$ is the radial distance of the $q$-th control point from the barycenter of $\Omega$, $\left(x_{\Omega}, y_{\Omega}\right),($ Fig. 2)

$$
\rho^{(q)}=\sqrt{\left(x_{\Omega}-\mathcal{C}_{x}^{(q)}\right)^{2}+\left(y_{\Omega}-\mathcal{C}_{y}^{(q)}\right)^{2}} .
$$

Moreover, $\mathcal{V}^{(q)}=\left(\mathcal{V}_{x}^{(q)}, \mathcal{V}_{y}^{(q)}\right)$ is the $q$-th $(q=1, \ldots, Q)$ spline virtual point

$$
\left\{\begin{array}{l}
\mathcal{V}_{x}^{(q)}=\frac{\mathcal{C}_{x}^{(q)}+\mathcal{C}_{x}^{(q+1)}}{2} \\
\mathcal{V}_{y}^{(q)}=\frac{\mathcal{C}_{y}^{(q)}+\mathcal{C}_{y}^{(q+1)}}{2}
\end{array}\right.
$$

and the condition $\mathcal{C}_{x}^{(Q+1)}=\mathcal{C}_{x}^{(1)}$ and $\mathcal{C}_{y}^{(Q+1)}=\mathcal{C}_{y}^{(1)}$ holds true so that $\partial \Omega(x, y)$ is a simplyconnected curve (Fig. 2).

Owing to such a parametric description of the scatterer support $\Omega$, the ISP solution is coded into the following $K=(4+Q) S b D-D o F$ s

$$
\underline{\xi}=\left\{x_{\Omega}, y_{\Omega}, \Re\left(\tau_{\Omega}\right), \Im\left(\tau_{\Omega}\right), \underline{\rho}\right\}
$$

where $\underline{\rho}=\left\{\rho^{(q)} ; q=1, \ldots, Q\right\}$. It is worth highlighting that such a parametric modeling yields also, as a by-product, a profitable regularization of the ISP by enforcing a physical a-priori knowledge on the unknown target.

As for the second task of the $P F$ block, the $I S P$ is re-formulated into an optimization/minimization one

$$
\underline{\xi}^{(o p t)}=\arg \left\{\min _{\underline{\xi}}[\Phi(\underline{\xi})]\right\}
$$

whose solution is the global minimum of the cost function $\Phi(\underline{\xi})$ set here to the normalized mismatch between measured, $\left\{\mathcal{S}^{(v)}\left(x_{m}, y_{m}\right) ; m=1, \ldots, M\right\}$, and estimated, $\left\{\widetilde{\mathcal{S}}^{(v)}\left(x_{m}, y_{m} \mid \underline{\xi}\right)\right.$; $m=1, \ldots, M\}$, scattered data

$$
\Phi(\underline{\xi})=\frac{\sum_{v=1}^{V} \sum_{m=1}^{M}\left|\mathcal{S}^{(v)}\left(x_{m}, y_{m}\right)-\widetilde{\mathcal{S}}^{(v)}\left(x_{m}, y_{m} \mid \underline{\xi}\right)\right|^{2}}{\sum_{v=1}^{V} \sum_{m=1}^{M}\left|\mathcal{S}^{(v)}\left(x_{m}, y_{m}\right)\right|^{2}} .
$$

In (15), $\underline{\mathcal{S}}^{(v)}(\underline{\xi})=\left\{\widetilde{\mathcal{S}}^{(v)}\left(x_{m}, y_{m} \mid \underline{\xi}\right) ; m=1, \ldots, M\right\}$ is the set of field data scattered in the observation domain $O$ from the scatterer, coded by $\underline{\xi}$, when illuminated by the $v$-th $(v=$ 
$1, \ldots, V)$ incident field, $\mathcal{I}^{(v)}$

\subsection{Data Computation (DC)}

In order to compute $\underline{\widetilde{\mathcal{S}}}^{(v)}(\underline{\xi})(v=1, \ldots, V)$, let us remember that it is the scattered data vector radiated by the $v$-th $(v=1, \ldots, V)$ equivalent current distribution $\underline{\mathcal{J}}^{(v)}(\underline{\xi})$ according to (6). Thus, $\underline{\xi}$ is first mapped into the corresponding $v$-th $(v=1, \ldots, V)$ equivalent current vector $\underline{\mathcal{J}}^{(v)}(\underline{\xi})$ whose generic $n$-th $(n=1, \ldots, N)$ entry is defined as

$$
\mathcal{J}_{n}^{(v)}(\underline{\xi}) \triangleq \mathcal{T}^{(v)}\left(x_{n}, y_{n} \mid \underline{\xi}\right) \tau\left(x_{n}, y_{n} \mid \underline{\xi}\right)
$$

Because of the spline-based representation of the unknown scattering profile of support $\Omega$, the relation between the $n$-th $(n=1, \ldots, N)$ contrast value $\tau\left(x_{n}, y_{n} \mid \underline{\xi}\right)$ and $\underline{\xi}$ is based on the Jordan curve theorem [48] that allows one to state whether a point $\left(x_{n}, y_{n}\right)$ belongs or not to the scatterer region $\Omega$ enclosed by the spline contour $\partial \Omega(x, y \mid \underline{\xi})$

$$
\tau\left(x_{n}, y_{n} \mid \underline{\xi}\right)=\left\{\begin{array}{cc}
\tau_{\Omega} & \text { if }\left(x_{n}, y_{n}\right) \in \partial \Omega(x, y \mid \underline{\xi}) \\
0 & \text { otherwise }
\end{array} .\right.
$$

On the other hand, the $n$-th $(n=1, \ldots, N)$ sample of the $v$-th $(v=1, \ldots, V)$ total field $\mathcal{T}^{(v)}\left(x_{n}, y_{n} \mid \underline{\xi}\right)$ is numerically derived from the $M o M$-discretized version of (1)

$$
\underline{\mathcal{T}}^{(v)}(\underline{\xi})=\left[\underline{\underline{I}}-\underline{\underline{G}}_{D} \underline{\underline{\tau}}(\underline{\xi})\right]^{-1} \underline{\mathcal{I}}^{(v)}
$$

where $\underline{\mathcal{I}}^{(v)}=\left\{\mathcal{I}^{(v)}\left(x_{n}, y_{n}\right) ; n=1, \ldots, N\right\}, \underline{\underline{\tau}}(\underline{\xi})=\operatorname{diag}\left\{\tau\left(x_{n}, y_{n} \mid \underline{\xi}\right) ; n=1, \ldots, N\right\}, \underline{\underline{I}}$ is the identity matrix, and $\underline{\underline{G}}_{D}$ is the $(N \times N)$ internal Green's operator whose $(n, p)$-th $(n$, $p=1, \ldots, N)$ entry is equal to $\left.\underline{\underline{G}}_{D}\right\rfloor_{n p}=j \frac{k_{0}^{2}}{4} \int_{D_{p}} \mathcal{H}_{0}^{(1)}\left(k_{0} \rho_{n}\right) d x^{\prime} d y^{\prime}$.

Once $\underline{\mathcal{J}}^{(v)}(\underline{\xi})(v=1, \ldots, V)$ has been obtained by substituting (18) and (17) in (16), the corresponding scattered field vector $\underline{\mathcal{S}}^{(v)}(\underline{\xi})(v=1, \ldots, V)$ is then computed through (6). 


\subsection{Cost Function Evaluation (CFE)}

To efficiently compute the data mismatch cost function (15), by avoiding the time-consuming call to the forward $(F W)$ solver in (5), the $L B E$ paradigm [43] is exploited to build a fast yet accurate surrogate of $\Phi(\underline{\xi}), \widehat{\Phi}(\underline{\xi})$, which is adaptively "reinforced" at each $i$-th $\left(i=1, \ldots, I_{S b D}\right)$ iteration of the optimization process performed in the SSE block (Sect. 3.4). More specifically, a Gaussian Process $(G P)$-based $D T$ [49][50] of $\Phi(\underline{\xi})$ is built at each $i$-th $\left(i=1, \ldots, I_{S b D}\right)$ iteration of the optimization, $\widehat{\Phi}_{i}(\underline{\xi})$, from a training set of $S_{i}$ known input/output (I/O) pairs according to the following "three-step" strategy leveraging on the interconnections among all SbD functional blocks (Fig. 1):

- Input-Space Reduction - Input the minimum set of $K$ highly-informative $S b D$-DoFs (13), which univocally describe the $I S P$ solution $\underline{\xi}$, from the $P F$ block (Sect. 3.1);

- Input-Space Representative Sampling - Build the smallest size $i$-th $\left(i=1, \ldots, I_{S b D}\right)$ training set

$$
\Lambda_{i}=\left\{\left[\underline{\xi}^{(s)}, \Phi\left(\underline{\xi}^{(s)}\right)\right] ; s=1, \ldots, S_{i}\right\}
$$

of $S_{i} I / O$ pairs to suitably represent the $K$-dimensional input space. It means that for each $s$-th $\left(s=1, \ldots, S_{i}\right)$ sample, $\underline{\xi}^{(s)}$, decoded with the $D C$ block, $\Phi\left(\underline{\xi}^{(s)}\right)$ is computed with a $F W$ solver. At the initialization $(i=0)$, the $\left.S_{i}\right\rfloor_{i=0}$ samples are selected according to the Latin Hypercube Sampling (LHS) strategy (see Appendix I) to uniformly explore the $S b D-D o F$ s thanks to its "input space filling" property [51], while new $I / O$ pairs are adaptively selected in the SSE block and added to the training set of the previous iteration, $\Lambda_{i-1}$, to build the $i$-th $\left(i=1, \ldots, I_{S b D}\right)$ training set, $\Lambda_{i}$, otherwise (i.e., $\left.1 \leq i \leq I_{S b D}\right)$;

- DT Generation - Starting from the $i$-th $\left(i=1, \ldots, I_{S b D}\right)$ training set, $\Lambda_{i}$, define the $i$-th $\left(i=1, \ldots, I_{S b D}\right) G P$ predictor [49][[50] of $\Phi(\underline{\xi}), \widehat{\Phi}_{i}(\underline{\xi})$, as follows

$$
\widehat{\Phi}_{i}(\underline{\xi})=\chi_{i}+\left[\underline{r}_{i}(\underline{\xi})\right]^{T} \underline{\underline{R}}_{i}^{-1}\left[\underline{\Phi}_{i}-\underline{1}_{i} \chi_{i}\right]
$$


where $\chi_{i}$ is a scalar term given by

$$
\chi_{i} \triangleq \frac{\underline{1}_{i}^{T} \underline{\underline{R}}_{i}^{-1} \underline{\Phi}_{i}}{\underline{1}_{i}^{T} \underline{\underline{R}}_{i}^{-1} \underline{1}_{i}}
$$

$\cdot^{T}$ being the transpose operator, $\underline{r}_{i}(\underline{\xi})$ is a $\left(S_{i} \times 1\right)$-dimensional vector whose $s$-th entry is equal to

$$
r_{i}\left(\underline{\xi}^{(s)}, \underline{\xi}\right)=\prod_{k=1}^{K} \exp \left(-\gamma_{i, k}\left|\xi_{k}^{(s)}-\xi_{k}\right|^{\beta_{i, k}}\right)
$$

$\underline{\underline{R}}_{i}$ is the $\left(S_{i} \times S_{i}\right)$ correlation matrix of $\Lambda$ whose $(s, u)$-th $\left(s, u=1, \ldots, S_{i}\right)$ element is $r_{i}\left(\underline{\xi}^{(s)}, \underline{\xi}^{(u)}\right)(\underline{22}), \underline{\Phi}_{i}=\left[\Phi\left(\underline{\xi}^{(s)}\right) ; s=1, \ldots, S_{i}\right]^{T}$, and $\underline{1}_{i}$ is a $\left(S_{i} \times 1\right)$ unitary column vector. Moreover, $\gamma_{i, k}$ and $\beta_{i, k}$ are the $k$-th $(k=1, \ldots, K)$ elements of the $G P$ hyperparameter vectors $\underline{\gamma}_{i}$ and $\underline{\beta}_{i}$, respectively, which are yielded from the maximization of the concentrated log-likelihood function [49]

$$
\Gamma\left(\underline{\gamma}_{i}, \underline{\beta}_{i}\right)=-\frac{1}{2}\left\{S_{i} \times \ln \left(\nu_{i}^{2}\right)+\ln \left[\operatorname{det}\left(\underline{\underline{R}}_{i}\right)\right]\right\}
$$

where

$$
\nu_{i} \triangleq \frac{1}{S_{i}}\left[\left(\underline{\Phi}_{i}-\underline{1}_{i} \chi_{i}\right)^{T} \underline{\underline{R}}_{i}^{-1}\left(\underline{\Phi}_{i}-\underline{1}_{i} \chi_{i}\right)\right]
$$

$\ln ($.$) and \operatorname{det}($.$) being the natural logarithm and the matrix determinant operators.$

It is worth noticing that the choice of the $G P$ to build the $D T$ of $\Phi(\underline{\xi})$, unlike other regression strategies such as, for instance, the Support Vector Regression (SVR) [43], ensures an exact prediction of the actual value of the cost function when a trial solution, $\underline{\xi}$, coincides with a training sample, $\underline{\xi}^{(s)}$ (i.e., $\widehat{\Phi}_{i}\left(\underline{\xi}^{(s)}\right)=\Phi\left(\underline{\xi}^{(s)}\right) ; s=1, \ldots, S_{i}$ ). Moreover, it must be pointed out that the definition of the $i$-th $\left(i=1, \ldots, I_{S b D}\right) G P$ surrogate model, $\widehat{\Phi}_{i}(\underline{\xi})$ in (20) is based on the assumption that the actual value of the cost function, $\Phi(\underline{\xi})$, is the realization of a normallydistributed random variable with average value $\widehat{\Phi}_{i}(\underline{\xi})$ and variance [49] equal to

$$
\delta_{i}^{2}(\underline{\xi})=\nu_{i}^{2}\left[1-\left[\underline{r}_{i}(\underline{\xi})\right]^{T} \underline{\underline{R}}_{i}^{-1} \underline{\underline{r}}_{i}(\underline{\xi})+\frac{\left[1-\underline{1}_{i}^{T} \underline{\underline{R}}_{i}^{-1} \underline{\underline{r}}_{i}(\underline{\xi})\right]^{2}}{\underline{1}_{i}^{T} \underline{\underline{R}}_{i}^{-1} \underline{1}_{i}}\right] .
$$


This latter quantity provides an estimate of the reliability of the GP-based $D T$, greater values of $\delta_{i}^{2}(\underline{\xi})$ corresponding to a lower "reliability" of the associated prediction $\widehat{\Phi}_{i}(\underline{\xi})$. Indeed, the value of $\delta_{i}^{2}(\underline{\xi})$ depends on $\underline{r}_{i}(\underline{\xi})(\underline{25})$, which in turn is related to the $\left(\underline{\gamma}_{i}, \underline{\beta}_{i}\right)$-weighted distance between $\underline{\xi}$ and the $s$-th $\left(s=1, \ldots, S_{i}\right)$ training sample, $\underline{\xi}^{(s)}(22)$. Thus, if $\underline{\xi}$ is very far from all the $S_{i}$ training samples, $\left\{\underline{\xi}^{(s)} ; s=1, \ldots, S_{i}\right\}$, then $\underline{r}_{i}(\underline{\xi}) \rightarrow \underline{0}$ and the uncertainty reaches its maximum (i.e., $\delta_{i}^{2}(\underline{\xi}) \rightarrow \nu_{i}^{2}$ ). On the contrary, the uncertainty is minimal in correspondence of the training samples [i.e., $\left.\delta_{i}^{2}\left(\underline{\xi}^{(s)}\right)=0\left(s=1, \ldots, S_{i}\right)\right]$ since $\left[\underline{r}_{i}\left(\underline{\xi}^{(s)}\right)\right]^{T} \underline{\underline{R}}_{i}^{-1} \underline{r}_{i}\left(\underline{\xi}^{(s)}\right)=$ $\underline{1}_{i}^{T} \underline{\underline{R}}_{i}^{-1} \underline{r}_{i}\left(\underline{\xi}^{(s)}\right)=1$. Finally, let us consider that, according to the GP theory [50], the actual value of the cost function $\Phi(\underline{\xi})$ fulfils at least to $95 \%$ probability [50] the following condition

$$
\mathcal{L}_{i}(\underline{\xi}) \leq \Phi(\underline{\xi}) \leq \mathcal{U}_{i}(\underline{\xi})
$$

$\mathcal{L}_{i}(\underline{\xi})$ and $\mathcal{U}_{i}(\underline{\xi})$ being the lower and the upper "confidence bounds", respectively, defined as

$$
\left\{\begin{array}{l}
\mathcal{L}_{i}(\underline{\xi})=\widehat{\Phi}_{i}(\underline{\xi})-2 \delta_{i}(\underline{\xi}) \\
\mathcal{U}_{i}(\underline{\xi})=\widehat{\Phi}_{i}(\underline{\xi})+2 \delta_{i}(\underline{\xi})
\end{array}\right.
$$

so that $\mathcal{L}_{i}\left(\underline{\xi}^{(s)}\right)=\mathcal{U}_{i}\left(\underline{\xi}^{(s)}\right)=\widehat{\Phi}_{i}\left(\underline{\xi}^{(s)}\right)=\Phi\left(\underline{\xi}^{(s)}\right)\left(s=1, \ldots, S_{i}\right)$

\subsection{Solution Space Exploration (SSE)}

To explore in a smart way the $K$-dimensional $S b D$ solution space for solving the non-linear $I S P$, nature-inspired $E A$ s are the most suitable candidates to effectively implement such a task without requiring, unlike $D O$ s, the differentiation of the data mismatch cost function (15) [32]. However, a "bare" integration of an $E A-G O$ with a forward solver $(F W)$ would imply an overall inversion time equal to

$$
\Delta t_{G O}=\left(P \times I_{G O}\right) \times \Delta t_{F W}
$$

$P$ and $\Delta t_{F W}$ being the number of trial solutions evolved through $I_{G O}$ iterations and the time of a single full-wave evaluation of (15), which clearly becomes unpractical in many applicative scenarios requiring a fast inversion. If a significant reduction of $P$ can be yielded with 
a minimum-dimensionality coding of the unknown scattering profile (e.g., the spline-based strategy in Sect. 3.1), it is not enough towards a computationally-competitive global inversion/optimization. In order to break down the computational burden required by the iterated (multi-agent) evaluation of (15) to comply with (7) by reducing $\Delta t_{G O}(28)$, there are two different strategies. The former is that of minimizing the number of iterations of the $E A$ to reach the global optimum $\underline{\xi}^{(o p t)}, I_{G O}$. Towards this end, it is mandatory to choose an $E A$ that provides a proper balance between exploration and exploitation to enable "hill-climbing" features for effectively escaping from local minima/false solutions as well as to guarantee a quick convergence towards the attraction basin of the global minimum of the cost function $\Phi(\underline{\xi})$. Accordingly, the Particle Swarm Optimization (PSO) algorithm [32] is chosen as a robust and effective evolutionary strategy particularly suitable for the exploration of the real-valued solution space of the $S b D-D o F s$ (13). During $I_{S b D}$ iterations, the $P S O$ processes a swarm of $P$ particles/agents, $\mathcal{A}=\left\{A^{(p)} ; p=1, \ldots, P\right\}$, by changing their velocities, $\mathcal{V}=\left\{\underline{\underline{L}}^{(p)} ; p=1, \ldots, P\right\}$, to evolve their positions in the solution space, $\mathcal{P}=\left\{\underline{\xi}^{(p)} ; p=1, \ldots, P\right\}$, until reaching the global optimum (i.e., $\underline{\xi}^{(o p t)}=\arg \left\{\min _{\underline{\xi}}[\Phi(\underline{\xi})]\right\}$ ).

The second method to shorten (28) is that of building a surrogate model in the CFE block (see Sect. 3.3) to replace the $F W$ solver during the optimization so that $\Delta t_{D T}^{t e s t} \ll \Delta t_{F W}$. However, the definition of a globally-accurate predictor would generally require a huge number of training samples $S[S \gg(P \times I)$ ], which not linearly depends on the number of scatterer descriptors, $K$, because of the so-called "curse-of-dimensionality" [42]. On the other hand, it is worth to consider that the $D T$ is required to predict the value of the cost function $\Phi(\underline{\xi})(15)$ for guiding the $G O$ search throughout the solution space with an accuracy adaptively enhanced and very high only in the attraction basin (i.e., in the proximity) of the global optimum. Owing to such considerations, a "collaborative" framework is implemented between the PSO, which is responsible of sampling the solution space with the swarm $\mathcal{A}$ of $P$ trial agents, and the $D T$ model based on the GP regression strategy [49][50] that gives not only a prediction of the cost function associated to each trial solution, $\widehat{\Phi}\left(\underline{\xi}^{(p)}\right)(p=1, \ldots, P)$, but also an estimate of its "degree of reliability", $\delta\left(\underline{\xi}^{(p)}\right)$. This latter is an additional information to be profitably exploited for identifying "promising" solutions for which the cost function (15) is expected to 
be lower than any previously-explored solution set. Moreover, the value $\delta$ can be used as a threshold for triggering adaptive refinements/reinforcements, obtained by simulating selected particles to enhance the accuracy only "where needed", of the predictor during the optimization loop. The resulting SSE block then works as follows:

1. Initialization $(i=0)$ - With the $C F E$ block (Sect. 3.3), build the initial training set of $S_{0} I / O$ pairs, $\Lambda_{0}=\left\{\left[\underline{\xi}^{(s)}, \Phi\left(\underline{\xi}^{(s)}\right)\right] ; s=1, \ldots, S_{0}\right\}$, to train the initial GP predictor $\widehat{\Phi}_{i}(\underline{\xi})$. Randomly initialize the positions of the swarm $\mathcal{A}_{0}$ of $P$ particles, $\mathcal{P}_{0}=$ $\left\{\underline{\xi}_{0}^{(p)} ; p=1, \ldots, P\right\}$, with random velocities, $\mathcal{V}_{0}=\left\{\underline{\iota}_{0}^{(p)} ; p=1, \ldots, P\right\}$, and set the personal best position of each $p$-th $(p=1, \ldots, P)$ particle to the initial one (i.e., $\left.\underline{\zeta}_{0}^{(p)}=\underline{\xi}_{0}^{(p)}\right)$;

2. SbD Optimization Loop $\left(i=1, \ldots, I_{S b D}\right)$

(a) Cost Function Prediction - For each $p$-th $(p=1, \ldots, P)$ particle of the current $i$-th swarm, $\mathcal{A}_{i}$, predict the values of $\Phi\left(\underline{\xi}_{i}^{(p)}\right), \mathcal{L}\left(\underline{\xi}_{i}^{(p)}\right)$, and $\mathcal{U}\left(\underline{\xi}_{i}^{(p)}\right)$, with the $i$-th $D T$ $\widehat{\Phi}_{i}(\underline{\xi})$;

(b) Particles Ranking - Determine the "best promising" $(B P)$ position of a particle of $\mathcal{A}_{i}$

$$
\underline{\xi}_{i}^{(B P)}=\arg \left\{\min _{p=1, \ldots, P}\left[\mathcal{L}_{i}\left(\underline{\xi}_{i}^{(p)}\right)\right]\right\}
$$

(c) DT Adaptive Updating - If $\mathcal{L}\left(\underline{\xi}_{i}^{(B P)}\right)<\min _{s=1, \ldots, S_{i}}\left[\Phi\left(\underline{\xi}^{(s)}\right)\right]$ perform the following operations, otherwise set $S_{i} \leftarrow S_{i-1}$ and $\Lambda_{i} \leftarrow \Lambda_{i-1}$ and jump to Step 2(d):

i. Exploit the $D C$ bock (Sect. 3.2) to derive the $v$-th $(v=1, \ldots, V)$ induced equivalent current, $\underline{\mathcal{J}}^{(v)}\left(\underline{\xi}_{i}^{(B P)}\right)$ from $\underline{\xi}_{i}^{(B P)}$, then compute the corresponding scattered field, $\underline{\widetilde{\mathcal{S}}}^{(v)}\left(\underline{\xi}_{i}^{(B P)}\right)$;

ii. Compute $\Phi\left(\underline{\xi}_{i}^{(B P)}\right)$ with $(\underline{15})$;

iii. Update the training set by adding the $B P$ training set sample, $\Lambda_{i}=\Lambda_{i-1} \cup$ $\left\{\underline{\xi}_{i}^{(B P)}, \Phi\left(\underline{\xi}_{i}^{(B P)}\right)\right\}$, and let $S_{i} \leftarrow\left(S_{i-1}+1\right) ;$

iv. Use the $C F E$ block (Sect. 3.3) to re-train the GP predictor using the updated/reinforced training information within $\Lambda_{i}$. 
(d) Personal Best Updating - Update the personal best position of each $p$-th $(p=$ $1, \ldots, P)$ particle, $\underline{\zeta}_{i}^{(p)}=\left\{\zeta_{i, k}^{(p)} ; k=1, \ldots, K\right\}$, according to the $S b D$-updating rules in Fig. 3(a);

(e) Global Best Updating - Update the global best, $\underline{\psi}_{i}=\left\{\psi_{i, k} ; k=1, \ldots, K\right\}$ according to the work-flow in Fig. 3(b);

(f) Convergence Check - Stop the optimization if $i=I_{S b D}$ and output the $S b D$ solution, set to the current global best swarm position, $\underline{\xi}^{(S b D)}=\underline{\psi}_{i=I_{S b D}}$, along with its pixelwise representation $\underline{\tau}^{(S b D)}=\left\{\tau^{(S b D)}\left(x_{n}, y_{n}\right) ; n=1, \ldots, N\right\}$ yielded from the $D C$ bock (Fig. 1 - Sect. 3.2). Otherwise, proceed to Step 2( $g$ );

(g) Velocities Updating - Update the velocity vector $\left(\mathcal{V}_{i} \rightarrow \mathcal{V}_{i+1}\right)$ by computing the $k$-th $(k=1, \ldots, K)$ component of the velocity of the $p$-th $(p=1, \ldots, P)$ particle of the swarm $\mathcal{A}_{i+1}$ according to the $P S O$ mechanism

$$
\iota_{i+1, k}^{(p)}=w \iota_{i, k}^{(p)}+\ell_{1} \varsigma_{1}\left(\xi_{i, k}^{(p)}-\zeta_{i, k}^{(p)}\right)+\ell_{2} \varsigma_{2}\left(\xi_{i, k}^{(p)}-\psi_{i, k}\right)
$$

where $\varsigma_{1}$ and $\varsigma_{2}$ are real random values within the interval $[0,1]$, the acceleration coefficients $\ell_{1}$ and $\ell_{2}$ are positive user-defined real values, and $w$ is the constant inertial weight;

(h) Swarm Updating - Update the position vector $\left(\mathcal{P}_{i} \rightarrow \mathcal{P}_{i+1}\right)$ by adding to the $k$-th $(k=1, \ldots, K)$ component of the current position of the $p$-th $(p=1, \ldots, P)$ particle of the swarm $\mathcal{A}_{i+1}$ the corresponding term of the velocity vector $\mathcal{V}_{i+1}$

$$
\xi_{i+1, k}^{(p)}=\xi_{i, k}^{(p)}+\iota_{i+1, k}^{(p)}
$$

then let $i \leftarrow(i+1)$ and go to Step 2(a).

It is worth pointing out that the SSE block implements a novel "time-constrained reinforced $P S O$ " strategy to allow the user to a-priori fulfil the CPU-time target (7) by properly setting the size $S_{0}$ of the initial training set, $\Lambda_{0}$, and the maximum number of $D T$ "reinforcements", $I_{S b D}$, performed during the global minimization of (15). Indeed, the total number of calls to 
the $F W$ solver during a $S b D$ inversion, thus the $S b D$ time cost, as well, is upper-bounded to

$S=\left(S_{0}+I_{S b D}\right)$ (2) so that a $S b D$ inversion turns out to be computationally advantageous with respect to a standard $G O$ solution when $S \ll\left(P \times I_{G O}\right)$, with a time saving equal to

$$
\Delta t_{s a v} \simeq\left(\frac{\left(P \times I_{G O}\right)-S}{\left(P \times I_{G O}\right)}\right)=\left(\frac{\left(P \times I_{G O}\right)-\left(S_{0}+I_{S b D}\right)}{\left(P \times I_{G O}\right)}\right) .
$$

\section{Performance Assessment}

This section is aimed at presenting a set of representative numerical and experimental results drawn from an extensive validation of the proposed $S b D$-based inversion method. Unless stated otherwise, a square investigation domain $D$ of side $L_{D}=2 \times \lambda$ has been probed by $V=18$ incident plane waves impinging from the $V$ angular directions $\left\{\varphi_{v} \triangleq 2 \pi \frac{(v-1)}{V} ; v=1, \ldots, V\right\}$. The scattered field samples have been collected at $M=18$ probing locations uniformly distributed on a circular observation domain $O$ of radius $\rho_{O}=3 \times \lambda$. As for the generation of the synthetic scattered field data, the $M o M$ solution of the $F W$ problem (1) (5) has been performed by partitioning the investigation domain into $N_{F W}=40 \times 40$ square sub-domains, while $N=20 \times 20$ pixel bases have been adopted in the inversion process to avoid the inverse crime (see [1] $\mathrm{p}$. 174). Moreover, an additive Gaussian noise has been added to the synthetically-generated data samples to test the robustness of the inversion to different signal-to-noise ratios (SNRs). Furthermore, owing to the stochastic nature of the $S b D$-based approach, a set of $\Upsilon=50$ random executions has been run for each inversion dataset to ensure the statistic meaningfulness of the results.

Concerning the imaging results/performance and besides the pictorial representation of the reconstruction in terms of color-maps of the dielectric profile of $D$, the accuracy of the data inversion is quantified by the error index

$$
\Xi=\frac{1}{N} \sum_{n=1}^{N} \frac{\left|\tau\left(x_{n}, y_{n}\right)-\widetilde{\tau}\left(x_{n}, y_{n}\right)\right|}{\tau\left(x_{n}, y_{n}\right)+1}
$$

\footnotetext{
(2) If $S \ll 10^{3}$ (Sect. 4), the overall time required to train $\left(\Delta t_{D T}^{\text {train }}\right)$ and to test $\left(\Delta t_{D T}^{\text {test }}\right)$ the $D T$ model can be neglected since $I_{S b D} \times \Delta t_{D T}^{\text {train }} \ll \Delta t_{F W}$ and $P \times I_{S b D} \times \Delta t_{D T}^{t \text { test }} \ll \Delta t_{F W}$ [42].
} 
where $\tau\left(x_{n}, y_{n}\right)$ and $\widetilde{\tau}\left(x_{n}, y_{n}\right)$ stand for the actual and the retrieved contrast value of the $n$-th $(n=1, \ldots, N)$ pixel $D_{n}\left(D_{n} \in D\right)$, respectively.

The first test case deals with the noiseless reconstruction of the scatting profile in Fig. 4(a) having contrast $\tau_{\Omega}=4.0$. The $S b D$-based inversion has been carried out by considering a spline description of the scatter with $Q=4$ control points ( $\Rightarrow K=8$ - Tab. I) and choosing, according to the guidelines in [32] a swarm size of $P=10$ particles, a constant inertial weight equal to $w=0.4$, and acceleration coefficients with values $\ell_{1}=\ell_{2}=2.0$. To investigate on the dependence of the prediction accuracy of the $D T$ of the $F W$ solver on the size of the initial training set $S_{0}$, a set of experiments has been run by varying the $S_{0} / K$ ratio and the adaptive generation of a fixed amount of $I_{S b D}=100$ additional training samples according to the $S S E$ procedure (Sect. 3.4). For each test, the normalized prediction error $\eta$

$$
\eta\left(S_{0} / K\right) \triangleq \frac{1}{P} \sum_{p=1}^{P} \frac{\left|\Phi\left(\underline{\xi}_{i=I_{S b D}}^{(p)}\right)-\widehat{\Phi}\left(\underline{\xi}_{i=I_{S b D}}^{(p)} \mid S_{0}\right)\right|}{\Phi\left(\underline{\xi}_{i=I_{S b D}}^{(p)}\right)}
$$

of the $G P$ surrogate trained with $S=\left(S_{0}+I_{S b D}\right)$ samples has been evaluated along with the time saving (32) with respect to a "bare" GO based on the standard PSO (i.e., a PSO-based inversion method that exploits the same spline-based coding, but that computes the cost function of each trial solution by solving the corresponding $F W$ problem with the $M o M$ ) run with equal swarm size, $P$, for the maximum number of iterations $\left(I_{G O}=I_{S b D}\right)$. As expected, the plot of $\eta$ and $\Delta t_{\text {sav }}$ versus $S_{0} / K$ (Fig. 5) indicates that the prediction accuracy improves widening the initial training set (e.g., $\left.\eta\right|_{S_{0} / K=1.25}=\left.27 \% \rightarrow \eta\right|_{S_{0} / K=20}=5 \%$ ), but the time saving reduces $\left(\left.\Delta t_{s a v}\right|_{S_{0} / K=1.25}=\left.89 \% \rightarrow \Delta t_{s a v}\right|_{S_{0} / K=20}=74 \%\right)$ albeit in a less evident way. The ratio $S_{0} / K=5\left(\left.\rightarrow \eta\right|_{S_{0} / K=5} \approx 7 \%\right.$ and $\left.\left.\Delta t_{s a v}\right|_{S_{0} / K=5}=86 \%\right)$ has been then chosen as the optimal trade-off threshold to fit (7). More specifically, the size of the initial and the final training datasets have been set here to $S_{0}=5 \times K=40$ and $S=140$, respectively, so that the total execution time of the $S b D$ is equal to that of a $D O$ method (i.e., $\Delta t_{S b D} \approx \Delta t_{D O}$ ), which is based on a standard implementation of the Conjugate Gradient $(C G)$ technique, running for $I_{D O}=$ 400 iterations [24]. By using such a setup, Figure 6 shows the evolution of the optimal value

\footnotetext{
${ }^{(3)}$ For fair comparisons, all inversions have been executed using non-optimized FORTRAN codes on a standard laptop equipped with 16 [GB] of RAM memory and an Intel(R) Core(TM) i5-8250U CPU @ 1.60 [GHz].
} 
of the cost function, $\Phi_{i} \triangleq \Phi\left(\underline{\psi}_{i}\right)$, during the $\operatorname{SSE}$ minimization $\left(i=1, \ldots, I_{S b D}\right)$. As it can be observed, $I_{S b D}=100$ iterations are enough to decrease the data mismatch of about two orders of magnitude (i.e., $\frac{\Phi_{I_{S b D}^{(S b D)}}^{(S b D)}}{\Phi_{0}^{(S b D}}=2.07 \times 10^{-2}$ - Fig. 6). Moreover, the values of $\Phi_{i}$ are very similar to those when applying a standard $G O$ (e.g., $\frac{\Phi_{I_{S b D}}^{(S b D)}}{\Phi_{I_{G O}}^{(G O)}}=1.03$ - Fig. 6). This proves the reliability of the $S b D$ algorithm to faithfully sample/explore the solution space looking for the global optimum even though guided by a $D T$ model of the $F W$ solver. For completeness, the behavior of the $D O$ minimization is reported, as well. To better understand the optimization performance of the three inversion approaches, Figure 7 shows the $2-D$ parametric representation of the functional described by the following equation

$$
\Phi(a, b)=\Phi\left\{b \times\left[(a+1) \times \underline{\xi}^{(1)}-a \times \underline{\xi}^{(a c t)}\right]+(b-1) \times a \times \underline{\xi}^{(2)}\right\}
$$

in the ranges $-1.5 \leq a \leq 0.5$ and $-0.5 \leq b \leq 1.5$ when setting $\underline{\xi}^{(1)}=\xi^{(S b D)}$ and $\underline{\xi}^{(2)}=\underline{\xi}^{(D O)}$ [Fig. 7(a)] or $\underline{\xi}^{(2)}=\underline{\xi}^{(G O)}$ [Fig. 7(b)], $\underline{\xi}^{(a c t)}$ being the actual solution (4). The landscape in Fig. 7(a) proves that the $D O$ solution is trapped into a local minimum of the cost function (i.e., a false solution for the inversion) without any possibility to escape from such a "wrong" valley. This is even more evident by looking at the plot of the cost function along the 1-D cut of the solution space passing through $\underline{\xi}^{(D O)}$ and the actual solution [i.e., $\left.\Phi(a, b)\right|_{a=-1} ^{\xi^{(2)}=\underline{\xi}^{(D O)}}$ - Fig. $7(c)$ ] (5). Otherwise, the $S b D$ solution $\underline{\xi}^{(S b D)}$ belongs to the "attraction basin" of the actual solution $\underline{\xi}^{(a c t)}$ analogously to the $G O$ solution $\underline{\xi}^{(G O)}$ [Figs. 7(b)-7(c)]. Such outcomes are confirmed by the corresponding reconstructions in Figs. $4(b)-4(d)$. Indeed, the DO inversion is unsatisfactory and remarkably worse than the $S b D$ one, as quantified by the integral errors (i.e., $\frac{\left.\Xi\right|_{D O}}{\left.\Xi\right|_{S b D}}=51.3$ Fig. 8), even though the execution time of the two iterative minimizations is approximately the same $\left(\left.\Delta t\right|_{D O}=480\right.$ [sec] vs. $\left.\Delta t\right|_{S b D}=490$ [sec] - Fig. 8). Furthermore, the computational efficiency of the $S b D$ is disruptive when compared to the standard $G O$ since $\frac{\left.\Delta t\right|_{S b D}}{\left.\Delta t\right|_{G O}}=0.14(\Rightarrow$ $\Delta t_{s a v}=86 \%$ - Fig. 8), while yielding the same accuracy (i.e., $\frac{\left.\Xi\right|_{G O}}{\left.\Xi\right|_{S b D}}=0.99$ - Fig. 8).

\footnotetext{
${ }^{(4)}$ According to (35), it can be easily verified that $\Phi(-1,1)=\Phi\left(\underline{\xi}^{(a c t)}\right), \Phi(0,1)=\Phi\left(\underline{\xi}^{(1)}\right)$, and $\Phi(-1,0)=$ $\Phi\left(\underline{\xi}^{(2)}\right)$.

${ }^{(5)}$ It is worth pointing out that a standard definition of the DO-DoFs, i.e., $\underline{\xi}^{(D O)}=$ $\left\{\mathcal{T}^{(v)}\left(x_{n}, y_{n}\right) ; \tau\left(x_{n}, y_{n}\right) ; v=1, \ldots, V ; n=1, \ldots, N\right\}$, has been adopted according to the reference literature on gradient-based local search algorithms [8].
} 
In order to assess the robustness of the reconstruction process to blurred/corrupted data, Figure 9(a) compares the behavior of the cost function for the $S b D$ and the $G O$ optimizations when varying the $S N R$ of the scattered field samples. As expected, the data matching gets worse as the noise increases from $S N R=20[\mathrm{~dB}]$ up to $S N R=5[\mathrm{~dB}]$ [i.e., $\left.\Phi\left(\underline{\xi}^{(S b D)}\right)\right|_{S N R=20[\mathrm{~dB}]}=$ $6.84 \times 10^{-2}$ vs. $\left.\Phi\left(\underline{\xi}^{(S b D)}\right)\right|_{S N R=10[\mathrm{~dB}]}=1.32 \times 10^{-1}$ vs. $\left.\Phi\left(\underline{\xi}^{(S b D)}\right)\right|_{S N R=5[\mathrm{~dB}]}=2.70 \times 10^{-1}$ - Fig. $9(a)$ ], but the $S b D$ still performs as the $G O$, while reducing the inversion time $\left[\Delta t_{\text {sav }}=\right.$ $86 \%$ - Fig. $9(b)$ ], despite the need of predicting the cost function values starting from non-ideal (blurred) data. The reliability of the $S b D$ in emulating a $G O$ when exploring highly-nonlinear solution spaces is confirmed by the comparison of the corresponding reconstruction errors [Fig. $9(b)$ ], which are almost identical whatever the amount of noise and, always, significantly lower than the $D O$ ones. As a matter of fact, the $D O$ is unable either to find a satisfactory reconstruction [Figs. 11 $(g)-11(i)$ ] or to localize the attraction basin of the global optimum [Fig. 10(a), Fig. 10(c), and Fig. 10(e)]. It is also worth noticing that the $S b D$ is effective even under very harsh operative conditions (e.g., $S N R=5[\mathrm{~dB}]$ ) as confirmed pictorially in Fig. 11(c) and quantitatively by the value of the error index [i.e., $\left.\Xi\right|_{S N R=5[\mathrm{~dB}]} ^{S b D}=5.6 \times 10^{-2}$ - Fig. $\left.9(b)\right]$.

The next set of results are concerned with the dependence of the data inversion on the contrast value of the scatterer, $\tau_{\Omega}$, still considering the extremely challenging scattering environment with $S N R=5[\mathrm{~dB}]$. Figure 12(a) gives some indications on the iterative minimization of the cost function. As expected, the weaker the scatterer more effective is the optimization process as denoted by the smaller and smaller values of the cost function at the convergence [i.e., $\left.\Phi\left(\underline{\xi}^{(S b D)}\right)\right|_{\tau_{\Omega}=1}=2.54 \times 10^{-1},\left.\Phi\left(\underline{\xi}^{(S b D)}\right)\right|_{\tau_{\Omega}=2}=2.57 \times 10^{-1}$, and $\left.\Phi\left(\underline{\xi}^{(S b D)}\right)\right|_{\tau_{\Omega}=10}=$ $5.72 \times 10^{-1}$ being $\left.\Phi\left(\underline{\xi}^{(G O)}\right)\right|_{\tau_{\Omega}=1}=2.50 \times 10^{-1},\left.\Phi\left(\underline{\xi}^{(G O)}\right)\right|_{\tau_{\Omega}=2}=2.56 \times 10^{-1}$, and $\left.\left.\Phi\left(\underline{\xi}^{(G O)}\right)\right|_{\tau_{\Omega}=10}=5.56 \times 10^{-1}\right]$. This implies that the reconstruction quality decreases as $\tau_{\Omega}$ increases [Fig. 12(a) and Fig. 13]. However, it has to be observed that the performance of the $G O$-based methods are significantly better than those from the $D O$, which results unable to handle high contrasts [e.g., $\tau_{\Omega}=10$ - Fig. 13(i)] that cause high non-linearities.

The second test case is related to a more complex scatterer profile [Fig. $16(a)$ ] with $\tau_{\Omega}=4.0$ and described by a larger number of spline control points $(Q=8)$, thus a greater dimensionality (i.e., $K=12$ - Tab. I) of the solution space. Therefore, a larger initial training set has been 
chosen to keep the optimal setup of the $S_{0} / K$ ratio (i.e., $S_{0}=5 \times K=60$ ), while the number of $S b D$ iterations has been reduced (i.e., $I_{S b D}=I_{G O}=80$ ) to fit the time constraint (7). Despite the smaller number of optimization iterations, the higher dimensionality, and the non-negligible noise level of the scattered data (i.e., $S N R=10[\mathrm{~dB}]$ ), the $S b D$ solution is very close to the $G O$ one [i.e., $\Phi\left(\underline{\xi}^{(S b D)}\right)=2.07 \times 10^{-1}$ vs. $\Phi\left(\underline{\xi}^{(G O)}\right)=1.79 \times 10^{-1}$ - Fig. 14(a)] and, unlike the $D O$, it belongs to the "attraction basin" of the actual solution [Figs. 14(b)-14(c)]. Consequently, the retrieved contrast distributions [Figs. 16(b)-16(d)] quite faithfully reproduce the actual one [Fig. 16(a)] with similar values of the reconstruction error and significantly smaller than those of the $D O$ (i.e., $\left.\Xi\right|_{S b D}=5.46 \times 10^{-2}$ vs. $\left.\Xi\right|_{G O}=4.54 \times 10^{-2}$ vs. $\left.\Xi\right|_{D O}=4.36 \times 10^{-1}$ - Fig. 15). On the other hand, the $C P U$-time of the $S b D$ inversion is remarkably lower that of the $G O$ (i.e., $\Delta t_{\text {sav }}=82.5 \%$ - Fig. 15 ) and very close to the $D O$.

The flexibility of the adopted minimum-dimensionality encoding of the unknown scattering profiles as well as the feasibility of representing doubly connected $(D C)$ contours/inhomogeneous objects is assessed in the third test case [Fig. 18(a)]. More in detail, the scatterer has been modeled with the following set of $K=11\left(\Rightarrow S_{0}=5 \times K=55, I_{S b D}=I_{G O}=85\right)$ descriptors

$$
\underline{\xi}_{D C}=\left\{x_{\Omega}, y_{\Omega}, \Re\left(\tau_{\Omega}^{(\text {out })}\right), \Im\left(\tau_{\Omega}^{(\text {out })}\right), \Re\left(\tau_{\Omega}^{(\text {int })}\right), \Im\left(\tau_{\Omega}^{(\text {int })}\right), \underline{\rho}^{(\text {out })}, v\right\}
$$

where the superscript $($ out $)[($ int $)]$ refers to the outer [internal] contour $\partial \Omega^{(\text {out })}\left[\partial \Omega^{(i n t)}\right]$, while $0<v<1$ is the scale factor between the two borders, the $q$-th $(q=1, \ldots, Q ; Q=4)$ control point of $\partial \Omega^{(i n t)}$ [i.e., $\left.\underline{\rho}^{(i n t)}=\left\{\rho^{(q, i n t)} ; q=1, \ldots, Q\right\}\right]$ being $\rho^{(q, i n t)}=v \rho^{(q, o u t)}$ (Tab. I). The outcomes from such a benchmark are summarized in Fig. 17(a) in terms of reconstruction errors and execution time. Once again, these results confirm the superior trade-off between computational efficiency and effectiveness of the $S b D$ method over the $G O$ and the $D O$ ones. As for the retrieved contrast, Figure 18 shows that the $S b D$ reconstruction provides a reliable estimation of both the object shape and the contrast value $\left(\tau_{\Omega}^{(\text {out })}=3, \tau_{\Omega}^{(\text {int })}=1, v=0.6\right.$ - Tab. I) well detecting the presence of a "hole" $\left[\left.\Xi\right|_{S b D}=3.30 \times 10^{-2}\right.$ - Fig. 18(c) vs. Fig. 18(a)]. Similar outcomes can be drawn [Fig. 17(b)] for the inhomogeneous profile in Fig. 18(b) $\left(\tau_{\Omega}^{(\text {out })}=2, \tau_{\Omega}^{(\text {int })}=4, v=0.4\right.$ - Tab. I), the dielectric profile inferred by the $S b D$ being shown in Fig. $18(d)\left[\left.\Xi\right|_{S b D}=5.13 \times 10^{-2}\right.$ - Fig. 17(b)]. 
The extension to multiple objects $(M O)$ is dealt with in the Test Case \#4 where two disconnected scatterers have been considered. In this case, the $K=16$ unknowns are

$$
\underline{\xi}_{M O}=\left\{x_{\Omega}^{(1)}, y_{\Omega}^{(1)}, x_{\Omega}^{(2)}, y_{\Omega}^{(2)}, \Re\left(\tau_{\Omega}^{(1)}\right), \Im\left(\tau_{\Omega}^{(1)}\right), \Re\left(\tau_{\Omega}^{(2)}\right), \Im\left(\tau_{\Omega}^{(2)}\right), \underline{\rho}^{(1)}, \underline{\rho}^{(2)}\right\},
$$

where the superscripts $(1) /(2)$ refer to the two disconnected spline contours $\partial \Omega^{(1)} / \partial \Omega^{(2)}(Q=$ 4 ), and the $S b D$ has been run for $I_{S b D}=I_{G O}=60$ iterations starting from a training set with $S_{0}=5 \times K=80 \mathrm{I} / \mathrm{O}$ pairs. Despite the higher complexity of the ISP problem at hand, also related to a larger dimension of the solution space as well as the non-negligible contrast of both scatterers $\left(\tau_{\Omega}^{(1)}=\tau_{\Omega}^{(2)}=4\right)$, the $S b D$ carefully images the investigation domain [i.e., $\frac{\left.\Xi\right|_{S b D}}{\left.\Xi\right|_{G O}}=1.05$ - Fig. $20(b)$ vs. Fig. $20(c)$ and $\frac{\left.\Xi\right|_{S b D}}{\left.\Xi\right|_{D O}}=8.9 \times 10^{-2}$ - Fig. $19(b)$ vs. Fig. $\left.19(d)\right]$ by reducing the inversion time of about $\Delta t_{\text {sav }}=76.7 \%$ (Fig. 19).

Finally (Test Case \#5), the $S b D$-based imaging method has been assessed against laboratorycontrolled experimental data. With reference to the data provided by the Institut Fresnel [52], the "FoamDielInt" scattering scenario has been selected as representative benchmark. It consists of a foam cylinder with diameter $8.0 \times 10^{-2}[\mathrm{~m}]$ and contrast $\tau_{\Omega}^{(o u t)}=0.45$ that embeds a smaller, $3.1 \times 10^{-2}[\mathrm{~m}]$ in diameter, and weaker, $\tau_{\Omega}^{(i n t)}=2.0$, dielectric cylinder [Fig. 21(a)]. The acquisition system was composed by $V=8$ ridged-horn antennas working at $f=2[\mathrm{GHz}]$ to probe a square investigation domain $D$ of side $L_{D}=0.2[\mathrm{~m}]$. The scattered data have been collected in $M=241$ uniformly-spaced locations on a circular observation domain $O$ with radius $\rho_{O}=1.67[\mathrm{~m}]$ [52]. Because of the topology of the object at hand, the exploration of the solution space defined by the $D o F \mathrm{~s}$ in (36) has been carried out by letting $S_{0}=55$ and $I_{S b D}=I_{G O}=85$ according to the previous examples. Figure 21(b) shows the retrieved contrast distribution. Similarly to the GO image [Fig. 21(c)], it is possible to detect the twolayers scatterer with a reliable estimation of the outer support of the object, $\partial \Omega^{(\text {out })}$, as well as to infer the presence of an inner scatterer/layer with higher permittivity. Once again, it turns out that it is possible to address the problem of local minima by exploiting the "hill-climbing" features of an $E A$-based multiple-agent approach, but solving the arising global minimization task with a remarkable time saving over a standard $G O$ implementation (i.e., $\Delta t_{\text {sav }}=83.5 \%$ ) by equalling the computational efficiency of the $D O$ [Fig. $21(d)]$. 


\section{Conclusions}

An innovative strategy has been proposed to address the computationally-efficient yet reliable solution of the fully non-linear ISP. The inversion method has been built by implementing the pillar concepts of the $S b D$ framework [42] to allow an effective exploration of the multi-modal landscape defined by the data mismatch cost function with the same time cost of a standard deterministic local search.

From a methodological point of view and to the best of the authors' knowledge, the key advances of this research work with respect to the state-of-the-art literature can be summarized as follows:

- a "smart" and flexible minimum-dimensionality encoding of complex-shaped scatterers yielded with a spline-based modeling of the scattering profile (Sect. 3.1), which not only "implements" a more favorable "operating environment" for the underlying $E A$-based $G O$ strategy, but it also alleviates the "curse-of-dimensionality" problem;

- the use of a $G P$-based $L B E$ approach for building a fast and accurate $D T$ of the timeconsuming $F W$ solver that predicts the data mismatch cost function associated to each trial solution, but also provides additional information on the associated "confidence level" of this latter;

- the setup of a collaborative framework between the EA mechanisms and the DT model that enables an effective exploration of the solution space, which is adaptively sampled at selected and promising points to increase the prediction accuracy of the DT model as well as to speed-up the converge towards the attraction basin of the global-optimum/actualsolution.

Moreover, the main outcomes from the numerical and experimental assessment (Sect. 4) are:

- the $S b D$-based inversion method is a reliable tool for reaching the attraction basin of the global optimum without being trapped into local-minima/false-solutions also when highly nonlinear cost functions/strong scatterers are at hand; 
- it exhibits the same computational efficiency of a $D O$, breaking - for the first time to the authors' best knowledge - the widely-diffused idea that solving an ISP with an EA-based tool is generally computationally unaffordable;

- the range of a reliable and effective application of the $S b D$ inversion method extends from weak to strong simple as well as complex and multiple objects in harsh environmental conditions, as well, subject to a suitable choice of the $S b D$ building blocks according to the "no-free lunch" theorems [53];

- the $S b D$ inversion is able to effectively and efficiently process synthetic as well as real laboratory-controlled scattering data.

Future works, beyond the scope of this paper, will be aimed at extending the proposed $S b D$ based method to other applicative contexts (e.g., NDT/NDE, GPR investigations, biomedical imaging, or food quality assessment) involving - for instance - differential formulations of the $I S P$ to embed the a-priori knowledge on a reference/healthy background scenario.

\section{Appendix I}

The LHS strategy is implemented through the following procedure:

- Uniformly divide the admissible range $\mathbb{A}_{k}=\left[\xi_{k}^{\min }, \xi_{k}^{\max }\right]$ of each $k$-th $(k=1, \ldots, K)$ DoF into $S_{i}$ intervals $\left\{\mathbb{I}_{k}^{(s)} ; s=1, \ldots, S_{i}\right\}$ such that $\mathbb{A}_{k}=\cup_{s=1, \ldots, S_{i}} \mathbb{I}_{k}^{(s)}$;

- For each $k$-th $(k=1, \ldots, K)$ variable, randomly choose one value $\varkappa_{k}^{(s)}$ within each $s$-th $\left(s=1, \ldots, S_{i}\right)$ interval, $\mathbb{I}_{k}^{(s)}$, and form the corresponding set $\mathbb{S}_{k}=\left\{\varkappa_{k}^{(s)} \in \mathbb{I}_{k}^{(s)} ; s=1, \ldots, S_{i}\right\} ;$

- Until $s=S_{i}$, form the $s$-th $K$-dimensional sample $\underline{\xi}^{(s)}\left(\underline{\xi}^{(s)}=\left\{\xi_{k}^{(s)} ; k=1, \ldots, K\right\}\right)$ by letting $\xi_{k}^{(s)}=\mathcal{R}\left(\mathbb{S}_{k}\right)(k=1, \ldots, K)$ where the operator $\mathcal{R}($.$) outputs the value of$ one randomly-chosen entry of $\mathbb{S}_{k}$, which is then removed from it. Update the index $s$ $[s \leftarrow(s+1)]$ and repeat. 


\section{Acknowledgements}

This work has been partially supported by the Italian Ministry of Education, University, and Research within the Program PRIN 2017 (CUP: E64I19002530001) for the Project CYBERPHYSICAL ELECTROMAGNETIC VISION: Context-Aware Electromagnetic Sensing and Smart Reaction (EMvisioning) (Grant no. 2017HZJXSZ) and benefited from the networking activities carried out within the Project "SPEED" (Grant No. 61721001) funded by National Science Foundation of China under the Chang-Jiang Visiting Professorship Program, the Project 'Inversion Design Method of Structural Factors of Conformal Load-bearing Antenna Structure based on Desired EM Performance Interval' (Grant no. 2017HZJXSZ) funded by the National Natural Science Foundation of China, and the Project 'Research on Uncertainty Factors and Propagation Mechanism of Conformal Loab-bearing Antenna Structure' (Grant No. 2021JZD003) funded by the Department of Science and Technology of Shaanxi Province within the Program Natural Science Basic Research Plan in Shaanxi Province. A. Massa wishes to thank E. Vico for her never-ending inspiration, support, guidance, and help.

This work has been submitted to the IEEE for possible publication. Copyright may be transferred without notice, after which this version may no longer be accessible.

\section{References}

[1] X. Chen, Computational Methods for Electromagnetic Inverse Scattering. Hoboken, NJ, USA: Wiley, 2018.

[2] A. Abubakar, P. M. van den Berg, and J. Mallorqui, "Imaging of biomedical data using a multiplicative regularized contrast source inversion method," IEEE Trans. Microw. Theory Techn., vol. 50, no. 7, pp. 1761- 1771, Jul. 2002.

[3] P. Mojabi and J. LoVetri, "Microwave biomedical imaging using the multiplicative regularized Gauss-Newton inversion,” IEEE Antennas Wireless Propag. Lett., vol. 8, pp. 645-648, Jul. 2009. 
[4] Y. Gao and R. Zoughi, "Millimeter wave reflectometry and imaging for noninvasive diagnosis of skin burn injuries," IEEE Trans. Instrum. Meas., vol. 66, no. 1, pp. 77-84, Jan. 2017.

[5] A. Afsari, A. M. Abbosh, and Y. Rahmat-Samii, "Modified Born iterative method in medical electromagnetic tomography using magnetic field fluctuation contrast source operator,' IEEE Trans. Microw. Theory Techn., vol. 67, no. 1, pp. 454-463, Jan. 2019.

[6] X. Song, M. Li, F. Yang, S. Xu, and A. Abubakar, "Study on joint inversion algorithm of acoustic and electromagnetic data in biomedical imaging," IEEE J. Multiscale Multiphys. Comput. Techn., vol. 4, pp. 2-11, 2019.

[7] T. Cui, W. C. Chew, A. A. Aydiner, and S. Chen "Inverse scattering of two-dimensional dielectric objects buried in a lossy earth using the distorted Born iterative method," IEEE Trans. Geosci. Remote Sens., vol. 39, no. 2, pp. 339-346, Feb. 2001.

[8] M. Salucci, G. Oliveri, and A. Massa, "GPR prospecting through an inverse scattering frequency-hopping multi-focusing approach,” IEEE Trans. Geosci. Remote Sens., vol. 53, no. 12, pp. 6573-6592, Dec. 2015.

[9] M. Salucci, L. Poli, and A. Massa, "Advanced multi-frequency GPR data processing for non-linear deterministic imaging," Signal Proc., vol. 132, pp. 306-318, Mar. 2017.

[10] M. Salucci, L. Poli, N. Anselmi and A. Massa, "Multifrequency particle swarm optimization for enhanced multiresolution GPR microwave imaging," IEEE Trans. Geosci. Remote Sens., vol. 55, no. 3, pp. 1305-1317, Mar. 2017.

[11] Z. Liu, C. Li, D. Lesselier, and Y. Zhong, "Fast full-wave analysis of damaged periodic fiber-reinforced laminates," IEEE Trans. Antennas Propag., vol. 66, no. 7, pp. 3540-3547, Jul. 2018.

[12] R. Zoughi, Microwave Nondestructive Testing and Evaluation. Amsterdam, The Netherlands: Kluwer, 2000. 
[13] S. Kharkovsky and R. Zoughi, "Microwave and millimeter wave nondestructive testing and evaluation - Overview and recent advances," IEEE Instrum. Meas. Mag., vol. 10, no. 2, pp. 26-38, Apr. 2007.

[14] S. Caorsi, A. Massa, and M. Pastorino, "A crack identification microwave procedure based on a genetic algorithm for nondestructive testing," IEEE Trans. Antennas Propag., vol. 49, no. 12, pp. 1812-1820, Dec. 2001.

[15] K. Xu, Y. Zhong, X. Chen, and D. Lesselier, "A fast integral equation-based method for solving electromagnetic inverse scattering problems with inhomogeneous background," IEEE Trans. Antennas Propag., vol. 66, no. 8, pp. 4228-4239, Aug. 2018.

[16] Y. Chu, K. Xu, Y. Zhong, X. Ye, T. Zhou, X. Chen, and G. Wang, "Fast microwave through wall imaging method with inhomogeneous background based on Levenberg-Marquardt algorithm," IEEE Trans. Microw. Theory Techn., vol. 67, no. 3, pp. 1138-1147, Mar. 2019.

[17] M. Fallahpour and R. Zoughi, "Fast 3-D qualitative method for through-wall imaging and structural health monitoring," IEEE Geosci. Remote Sens. Lett., vol. 12, no. 12, pp. $2463-$ 2467, Dec. 2015.

[18] J. LoVetri, M. Asefi, C. Gilmore, and I. Jeffrey, "Innovations in electromagnetic imaging technology: The stored-grain-monitoring case," IEEE Antennas Propag. Mag., vol. 62, no. 5, pp. 33-42, Oct. 2020.

[19] J. A. Tobon Vasquez, R. Scapaticci, G. Turvani, M. Ricci, L. Farina, A. Litman, M. R. Casu, L. Crocco, and F. Vipiana, "Noninvasive inline food inspection via microwave imaging technology: An application example in the food industry," IEEE Antennas Propag. Mag., vol. 62, no. 5, pp. 18-32, Oct. 2020.

[20] C. Occhiuzzi, N. D’Uva, S. Nappi, S. Amendola, C. Gialluca, V. Chiabrando, L. Garavaglia, G. Giacalone, and G. Marrocco, "Radio-frequency-identification-based intelligent packaging: Electromagnetic classification of tropical fruit ripening," IEEE Antennas Propag. Mag., vol. 62, no. 5, pp. 64-75, Oct. 2020. 
[21] W. Chew and Y. Wang, "Reconstruction of two-dimensional permittivity distribution using the distorted Born iterative method," IEEE Trans. Med. Imag., vol. 9, no. 2, pp. 218-225, Jun. 1990.

[22] W. Zhang, L. Li, and F. Li, "Multifrequency imaging from intensity-only data using the phaseless data distorted Rytov iterative method," IEEE Trans. Antennas Propag., vol. 57, no. 1, pp. 290-295, Jan. 2009.

[23] Y. Zhong, M. Lambert, D. Lesselier, and X. Chen, "A new integral equation method to solve highly nonlinear inverse scattering problems," IEEE Trans. Antennas Propag., vol. 64, no. 5, pp. 1788-1799, May 2016.

[24] R. Kleinman and P. van den Berg, "A contrast source inversion method," Inverse Probl., vol. 13, no. 6, pp. 1607-1620, Jul. 1997.

[25] P. M. van den Berg, A. Abubakar, and J. Fokkema, "Multiplicative regularization for contrast profile inversion," Radio Sci., vol. 38, no. 2, Apr. 2003.

[26] K. Xu, Y. Zhong, R. Song, X. Chen, and L. Ran, "Multiplicative- regularized FFT twofold subspace-based optimization method for inverse scattering problems," IEEE Trans. Geosci. Remote Sens., vol. 53, no. 2, pp. 841-850, Jun. 2015.

[27] X. Chen, "Subspace-based optimization method for solving inverse-scattering problems," IEEE Trans. Geosci. Remote Sens., vol. 48, no. 1, pp. 42-49, Jan. 2010.

[28] Y. Zhong and X. Chen, “Twofold subspace-based optimization method for solving inverse scattering problems," Inverse Probl., vol. 25, 085003, Jul. 2009.

[29] Y. Zhong and X. Chen, "An FFT twofold subspace-based optimization method for solving electromagnetic inverse scattering problems," IEEE Trans. Antennas Propag., vol. 59, no. 3, pp. 914-927, Mar. 2011.

[30] H. Harada, D. J. N. Wall, T. Takenaka, and M. Tanaka, "Conjugate gradient method applied to inverse scattering problem," IEEE Trans. Antennas Propag., vol. 43, no. 8, pp. 784-792, Aug. 1995. 
[31] M. Salucci, G. Oliveri, N. Anselmi, F. Viani, A. Fedeli, M. Pastorino, and A. Randazzo, “Three-dimensional electromagnetic imaging of dielectric targets by means of the multiscaling inexact-Newton method," J. Opt. Soc. Am. A, vol. 34, no. 7, pp. 1119-1131, 2017.

[32] P. Rocca, M. Benedetti, M. Donelli, D. Franceschini, and A. Massa, "Evolutionary optimization as applied to inverse scattering problems," Inverse Probl., vol. 25, no. 12, pp. 123003, Dec. 2009.

[33] M. Pastorino, "Stochastic optimization methods applied to microwave imaging: A review," IEEE Trans. Antennas Propag., vol. 55, no. 3, pp. 538-548, Mar. 2007.

[34] S. Goudos, Emerging Evolutionary Algorithms for Antennas and Wireless Communications. SciTech/IET, 2021 (ISBN-13: 978-1-78561-552-8).

[35] P. Rocca, G. Oliveri, and A. Massa, "Differential Evolution as applied to electromagnetics," IEEE Antennas Propag. Mag., vol. 53, no. 1, pp. 38-49, Feb. 2011.

[36] S. Caorsi, M. Donelli, D. Franceschini, and A. Massa, "A new methodology based on an iterative multiscaling for microwave imaging," IEEE Trans. Microw. Theory Tech., vol. 51, pp. 1162-1173, 2003.

[37] M. Donelli, D. Franceschini, P. Rocca, and A. Massa, "Three-dimensional microwave imaging problems solved through an efficient multi-scaling particle swarm optimization," IEEE Trans. Geosci. Remote Sens., vol. 47, no. 5, pp. 1467-1481, May 2009.

[38] A. Massa, D. Marcantonio, X. Chen, M. Li, and M. Salucci, "DNNs as applied to electromagnetics, antennas, and propagation - A review," IEEE Antennas Wireless Propag. Lett., vol. 18 , no. 11 , pp. 2225-2229, Nov. 2019.

[39] K. Xu, L. Wu, X. Ye, and X. Chen, "Deep learning-based inversion methods for solving inverse scattering problems with phaseless data," IEEE Trans. Antennas Propag., vol. 68, no. 11, pp. 7457-7470, Nov. 2020. 
[40] L. Li, L. G. Wang, F. L. Teixeira, C. Liu, A. Nehorai, and T. J. Cui, "DeepNIS: Deep neural network for nonlinear electromagnetic inverse scattering," IEEE Trans. Antennas Propag., vol. 67, no. 3, pp. 1819-1825, Mar. 2019.

[41] Y. Zhou, Y. Zhong, Z. Wei, T. Yin, and X. Chen, "An improved deep learning scheme for solving 2D and 3D inverse scattering problems," IEEE Trans. Antennas Propag., doi: 10.1109/TAP.2020.3027898.

[42] A. Massa and M. Salucci, "On the design of complex EM devices and systems through the System-by-Design paradigm - A framework for dealing with the computational complexity," IEEE Trans. Antennas Propag. (under review).

[43] A. Massa, G. Oliveri, M. Salucci, N. Anselmi, and P. Rocca, "Learning-by-examples techniques as applied to electromagnetics," J. Electromagn. Waves Appl., pp. 1-16, 2017.

[44] M. Salucci, N. Anselmi, S. Goudos, and A. Massa, "Fast design of multiband fractal antennas through a system-by-design approach for NB-IoT applications," EURASIP J. Wirel. Commun. Netw., vol. 2019, no. 1, pp. 68-83, Mar. 2019.

[45] G. Oliveri, M. Salucci, N. Anselmi and A. Massa, "Multiscale system-by-design synthesis of printed WAIMs for waveguide array enhancement," IEEE J. Multiscale Multiphysics Computat. Techn., vol. 2, pp. 84-96, 2017.

[46] G. Oliveri, A. Gelmini, A. Polo, N. Anselmi, and A. Massa, "System-by-design multiscale synthesis of task-oriented reflectarrays," IEEE Trans. Antennas Propag., vol. 68, no. 4, pp. 2867-2882, Apr. 2020.

[47] M. Salucci, L. Tenuti, G. Gottardi, A. Hannan, and A. Massa, "System-by-design method for efficient linear array miniaturisation through low-complexity isotropic lenses," Electron. Lett., vol. 55, no. 8, pp. 433-434, May 2019.

[48] M. Shimrat, "Algorithm 112: Position of point relative to polygon," Communications of the ACM, vol. 5, no. 8, p. 434, 1962. 
[49] A. I. J. Forrester, A. Sobester, and A. J. Keane, Engineering Design via Surrogate Modelling: A Practical Guide. Hoboken, N.J.: John Wiley \& Sons, 2008.

[50] D. R. Jones, M. Schonlau, and W.J. Welch, "Efficient global optimization of expensive black-box functions," J. Global Opt., vol. 13, pp. 455-492, 1998.

[51] S. S. Garud, I. A. Karimi, and M. Kraft, "Design of computer experiments: a review," Comput. Chem. Eng., vol. 106, pp. 71-95, May 2017.

[52] J. Geffrin, P. Sabouroux, and C. Eyraud, "Free space experimental scattering database continuation: experimental set-up and measurement precision," Inverse Probl., vol. 21, no. 6, pp. 117-130, Nov. 2005.

[53] D. H. Wolpert and W. G. Macready, "No free lunch theorems for optimization," IEEE Trans. Evol. Comput., vol. 1, no. 1, pp. 67-82, Apr. 1997. 


\section{FIGURE CAPTIONS}

- Figure 1. Block scheme of the $S b D$-based inversion method.

- Figure 2. Pictorial sketch of the spline-based scatterer modeling.

- Figure 3. $S b D$-SSE update rules for $(a)$ the personal best position of each $p$-th $(p=$ $1, \ldots, P)$ particle, $\underline{\zeta}_{i}^{(p)}$, and $(b)$ the global best, $\underline{\zeta}_{i}$, at the $i$-th iteration $\left(i=1, \ldots, I_{S b D}\right)$.

- Figure 4. Numerical Assessment (Test Case \#1: $\tau_{\Omega}=4.0, V=M=18$, Noiseless Data; $K=8)$ - Maps of $(a)$ the actual and $(b)-(d)$ retrieved contrast distributions with $(b)$ the $S b D,(c)$ the $G O$, and $(d)$ the $D O$ methods.

- Figure 5. Numerical Assessment (Test Case \#1: $\tau_{\Omega}=4.0, V=M=18$, Noiseless Data; $K=8$ ) - Prediction error of the $D T, \eta$, and time saving, $\Delta t_{\text {sav }}$, versus the ratio $S_{0} / K$ between the number of initial training samples $S_{0}$, and the number of unknowns/SbDDoFs, $K$.

- Figure 6. Numerical Assessment (Test Case \#1: $\tau_{\Omega}=4.0, V=M=18$, Noiseless Data; $K=8)$ - Evolution of the optimal value of the cost function, $\Phi_{i}$, versus the iteration index, $i$.

- Figure 7. Numerical Assessment (Test Case \#1: $\tau_{\Omega}=4.0, V=M=18$, Noiseless Data; $K=8$ ) - Plot of the functional (35) $(a)(b)$ in the ranges $-1.5 \leq a \leq 0.5$ and $-0.5 \leq b \leq 1.5$ when setting $\underline{\xi}^{(1)}=\underline{\xi}^{(S b D)}$ and $(a) \underline{\xi}^{(2)}=\underline{\xi}^{(D O)}$ or $(b) \underline{\xi}^{(2)}=\underline{\xi}^{(G O)}$ or $(c)$ along the lines passing through $\left(\underline{\xi}^{(S b D)}, \underline{\xi}^{(a c t)}\right),\left(\underline{\xi}^{(D O)}, \underline{\xi}^{(a c t)}\right)$, and $\left(\underline{\xi}^{(G O)}, \underline{\xi}^{(a c t)}\right)$.

- Figure 8. Numerical Assessment (Test Case \#1: $\tau_{\Omega}=4.0, V=M=18$, Noiseless Data; $K=8)$ - Values of the reconstruction error, $\Xi$, and total inversion time, $\Delta t$.

- Figure 9. Numerical Assessment (Test Case \#1: $\tau_{\Omega}=4.0, V=M=18 ; K=8$ ) Plot of (a) the evolution of the optimal value of the cost function, $\Phi_{i}$, versus the iteration index, $i$, and of $(b)$ the reconstruction error, $\Xi$, and the execution time, $\Delta t$, versus the $S N R$ value of the scattered data. 
- Figure 10. Numerical Assessment (Test Case \#1: $\tau_{\Omega}=4.0, V=M=18 ; K=8$ ) Plot of the functional (35) $(a)(b)$ in the ranges $-1.5 \leq a \leq 0.5$ and $-0.5 \leq b \leq 1.5$ when setting $\underline{\xi}^{(1)}=\underline{\xi}^{(S b D)}$ and $(a)(c)(e) \underline{\xi}^{(2)}=\underline{\xi}^{(D O)}$ or $(b)(d)(f) \underline{\xi}^{(2)}=\underline{\xi}^{(G O)}$ for noisy scattered data with $(a)(b) S N R=20[\mathrm{~dB}],(c)(d) S N R=10[\mathrm{~dB}]$, and $(e)(f) S N R=5$ $[\mathrm{dB}]$.

- Figure 11. Numerical Assessment (Test Case \#1: $\tau_{\Omega}=4.0, V=M=18 ; K=8$ ) Reconstructions of the contrast profile in $D$ obtained by $(a)-(c)$ the $S b D,(d)-(f)$ the $G O$, and $(g)-(i)$ the $D O$ when processing noisy data with $(a)(d)(g) S N R=20[\mathrm{~dB}],(b)(e)(h)$ $S N R=10[\mathrm{~dB}]$, and $(c)(f)(i) S N R=5[\mathrm{~dB}]$.

- Figure 12. Numerical Assessment (Test Case \#1: $V=M=18, S N R=5[\mathrm{~dB}] ; K=8$ ) - Plot of $(a)$ the evolution of the optimal value of the cost function, $\Phi_{i}$, versus the iteration index, $i$, and of $(b)$ the reconstruction error, $\Xi$, and the execution time, $\Delta t$, versus the value of the contrast of the scatterer, $\tau_{\Omega}$.

- Figure 13. Numerical Assessment (Test Case \#1: $V=M=18, S N R=5[\mathrm{~dB}] ; K=8$ ) - Reconstructions of the contrast profile in $D$ obtained by $(a)-(c)$ the $S b D,(d)-(f)$ the $G O$, and $(g)-(i)$ the $D O$ when the actual value of the contrast of the scatterer is $(a)(d)(g)$ $\tau_{\Omega}=1,(b)(e)(h) \tau_{\Omega}=2$, and $(c)(f)(i) \tau_{\Omega}=10$.

- Figure 14. Numerical Assessment (Test Case \#2: $\tau_{\Omega}=4.0, V=M=18, S N R=10$ [dB]; $K=12$ ) - Plot of ( $a$ ) the evolution of the optimal value of the cost function, $\Phi_{i}$, versus the iteration index, $i$, and color maps of the functional $(35)(c)(d)$ in the ranges $-1.5 \leq a \leq 0.5$ and $-1.5 \leq b \leq 0.5$ when setting $\underline{\xi}^{(1)}=\underline{\xi}^{(S b D)}$ and $(c) \underline{\xi}^{(2)}=\underline{\xi}^{(D O)}$ or (d) $\underline{\xi}^{(2)}=\underline{\xi}^{(G O)}$.

- Figure 15. Numerical Assessment (Test Case \#2: $\tau_{\Omega}=4.0, V=M=18, S N R=10$ $[\mathrm{dB}] ; K=12)$ - Values of the reconstruction error, $\Xi$, and total inversion time, $\Delta t$.

- Figure 16. Numerical Assessment (Test Case \#2: $\tau_{\Omega}=4.0, \mathrm{~V}=M=18, S N R=10$ $[\mathrm{dB}] ; K=12)$ - Maps of $(a)$ the actual and $(b)-(d)$ the retrieved contrast distributions with (b) the $S b D,(c)$ the $G O$, and $(d)$ the $D O$ methods. 
- Figure 17. Numerical Assessment (Test Case \#3: $V=M=18, S N R=10[\mathrm{~dB}]$; $K=11$ ) - Values of the reconstruction error, $\Xi$, and total inversion time, $\Delta t$, for the scattering scenario in Fig. 18(a) when $(a)\left(\tau_{\Omega}^{(\text {out })}=3, \tau_{\Omega}^{(\text {int })}=0\right)$ and $\left(\right.$ b) $\left(\tau_{\Omega}^{(\text {out })}=2\right.$, $\left.\tau_{\Omega}^{(i n t)}=4\right)$.

- Figure 18. Numerical Assessment (Test Case \#3: $V=M=18, S N R=10[\mathrm{~dB}]$; $K=11)$ - Maps of $(a)(b)$ the actual and $(b)(d)$ the $S b D$-retrieved contrast distributions when $(a)(c)\left(\tau_{\Omega}^{(\text {out })}=3, \tau_{\Omega}^{(\text {int })}=0\right)$ and $(b)(d)\left(\tau_{\Omega}^{(\text {out })}=2, \tau_{\Omega}^{(\text {int })}=4\right)$.

- Figure 19. Numerical Assessment (Test Case \#4: $\tau_{\Omega}^{(1)}=\tau_{\Omega}^{(2)}=4, V=M=18$, $S N R=10[\mathrm{~dB}] ; K=16)$ - Values of the reconstruction error, $\Xi$, and total inversion time, $\Delta t$.

- Figure 20. Numerical Assessment (Test Case \#4: $\tau_{\Omega}^{(1)}=\tau_{\Omega}^{(2)}=4, V=M=18$, $S N R=10[\mathrm{~dB}] ; K=16)$ - Maps of $(a)$ the actual and $(b)-(d)$ the retrieved contrast distributions with $(b)$ the $S b D,(c)$ the $G O$, and $(d)$ the $D O$ methods.

- Figure 21. Experimental Assessment (Test Case \#5: $f=2[\mathrm{GHz}], \tau_{\Omega}^{(\text {out })}=0.45$, $\left.\tau_{\Omega}^{(i n t)}=2, V=8, M=241 ; K=11\right)$ - Maps of $(a)$ the actual "FoamDielInt" [52] and (b)-(d) the retrieved contrast distributions with $(b)$ the $S b D,(c)$ the $G O$, and $(d)$ the $D O$ methods.

\section{TABLE CAPTIONS}

- Table I. Performance Assessment ( $V=M=18$ ) - Test cases description. 


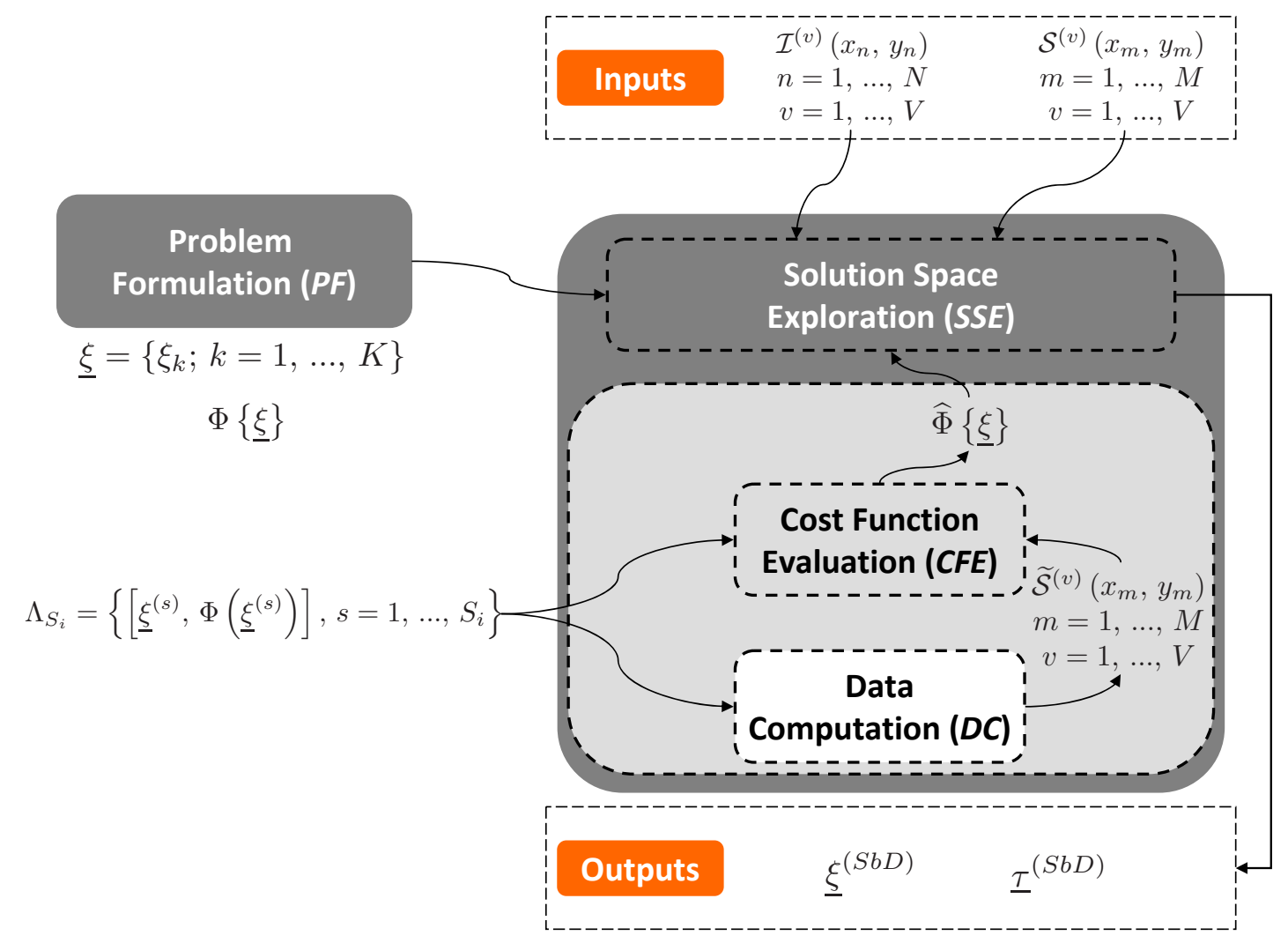

Fig. 1 - M. Salucci et al., "Learned Global Optimization ...” 


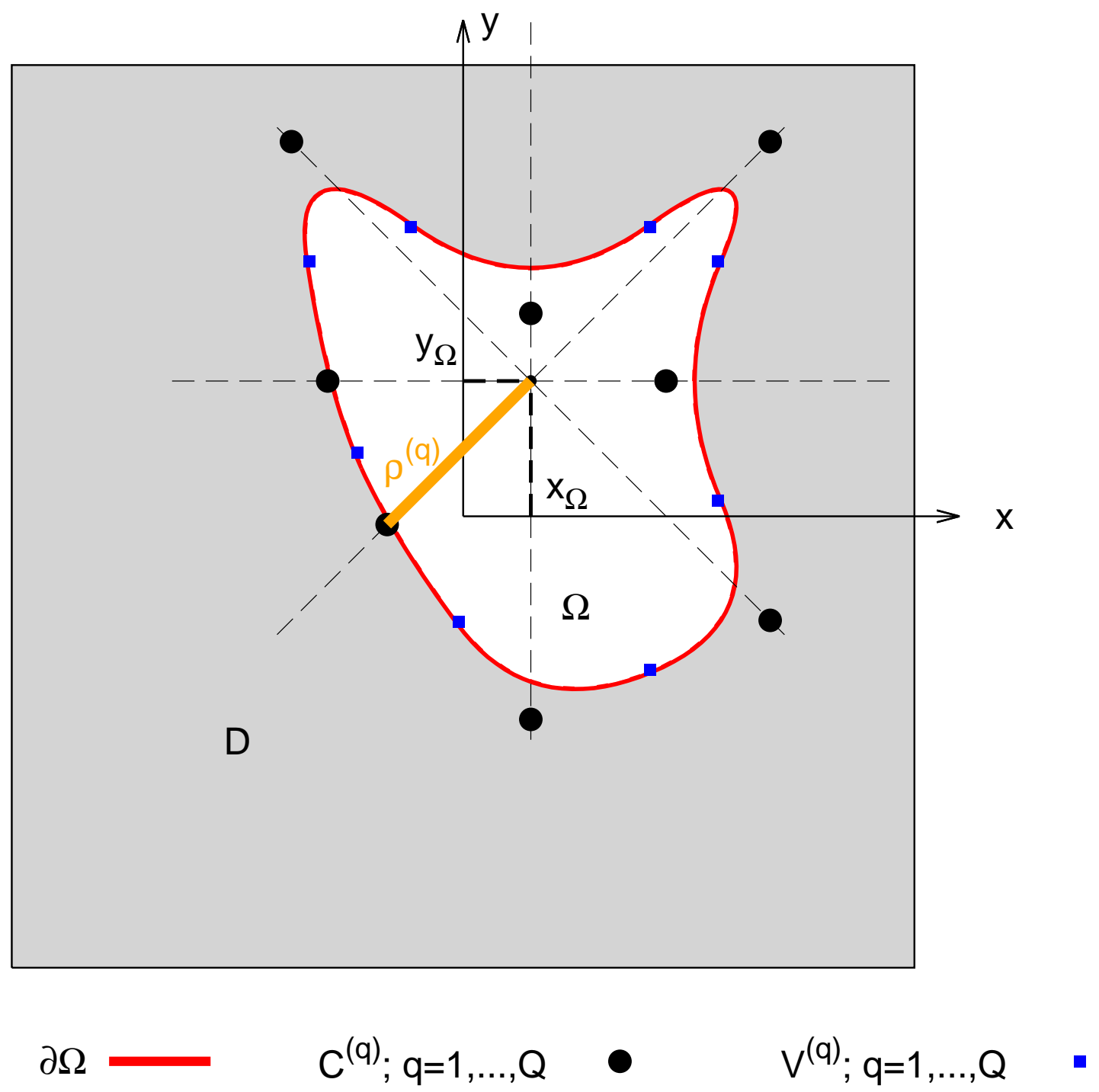

Fig. 2 - M. Salucci et al., "Learned Global Optimization ..." 


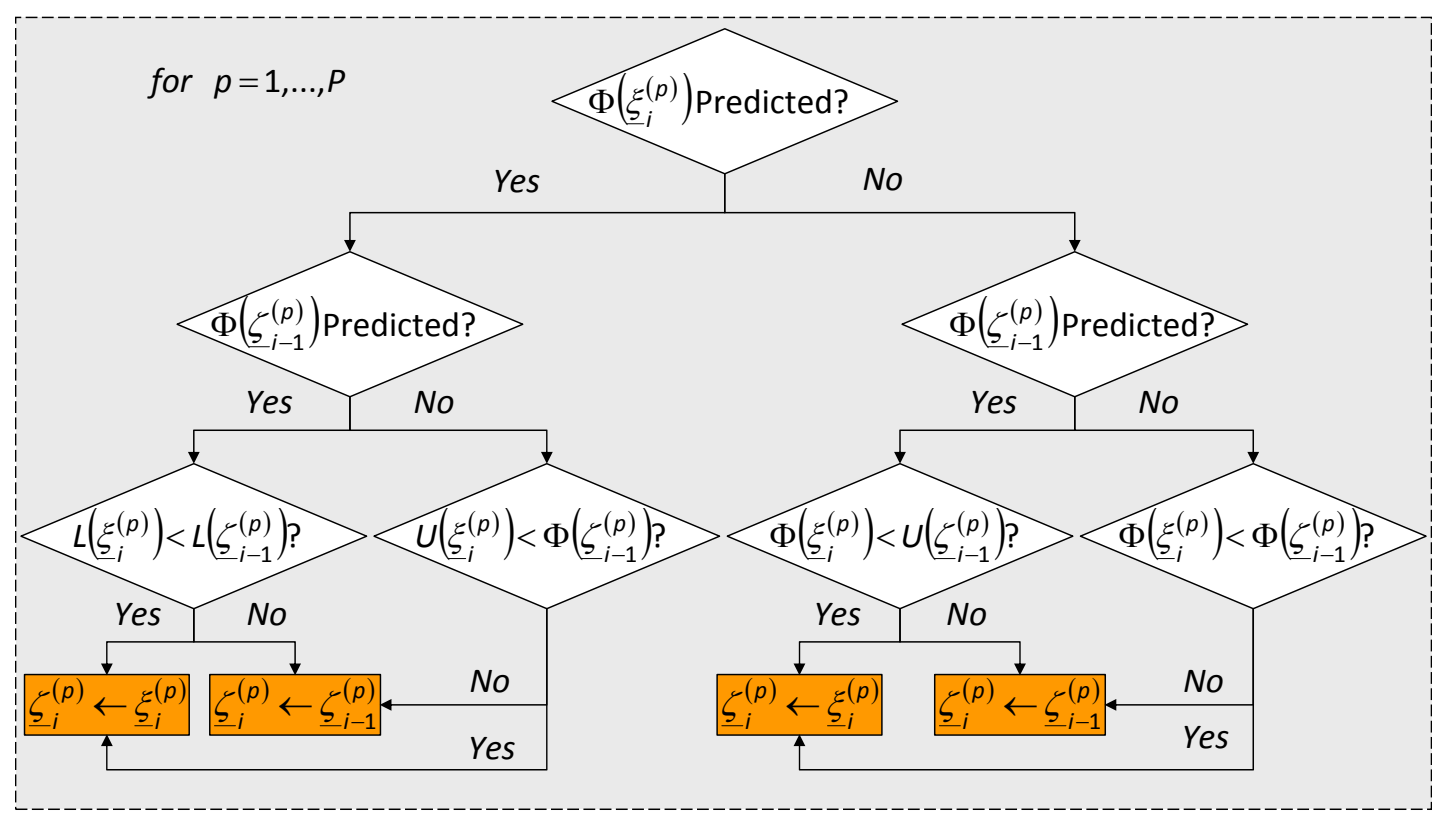

(a)

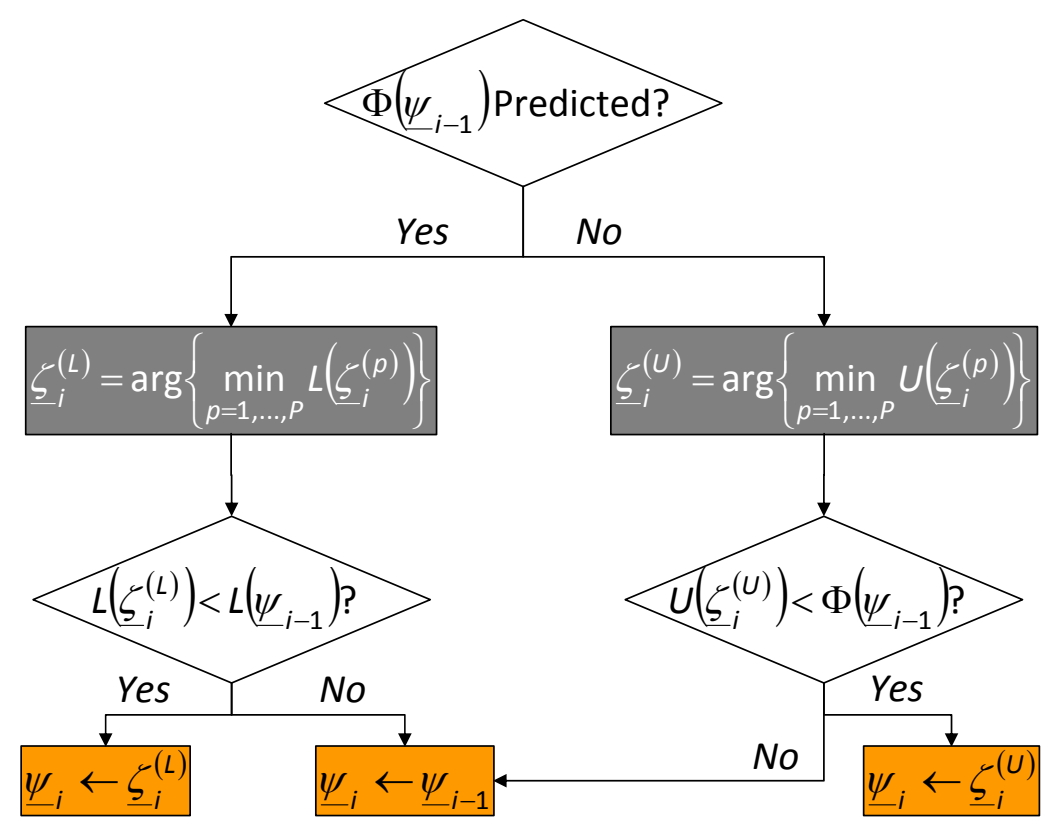

(b)

Fig. 3 - M. Salucci et al., "Learned Global Optimization ...” 


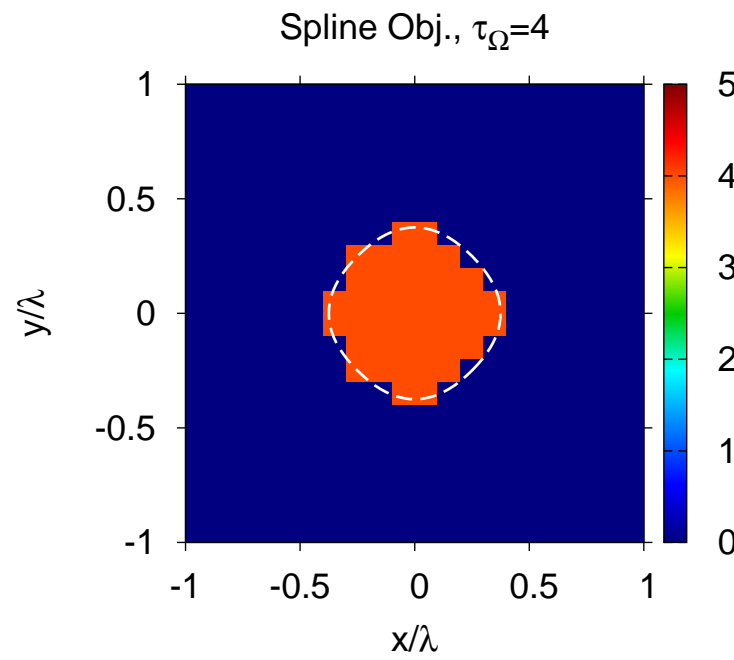

(a)

Spline Obj., $\tau_{\Omega}=4$ - GO - Noiseless Data

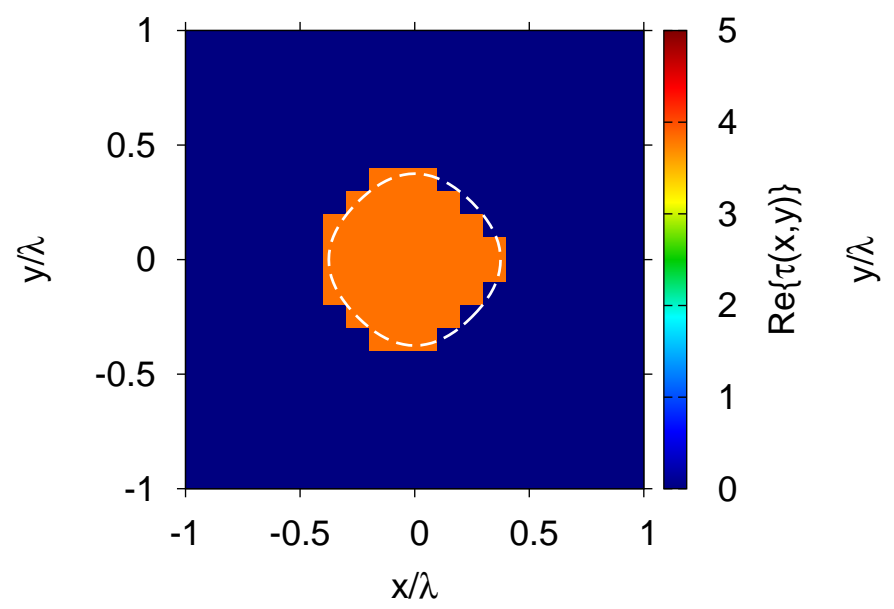

(c)

Spline Obj., $\tau_{\Omega}=4$ - SbD - Noiseless Data

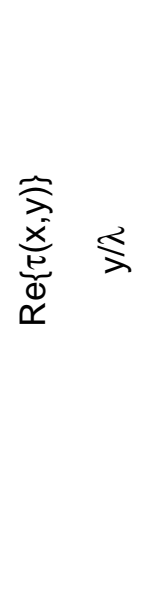

$(b)$

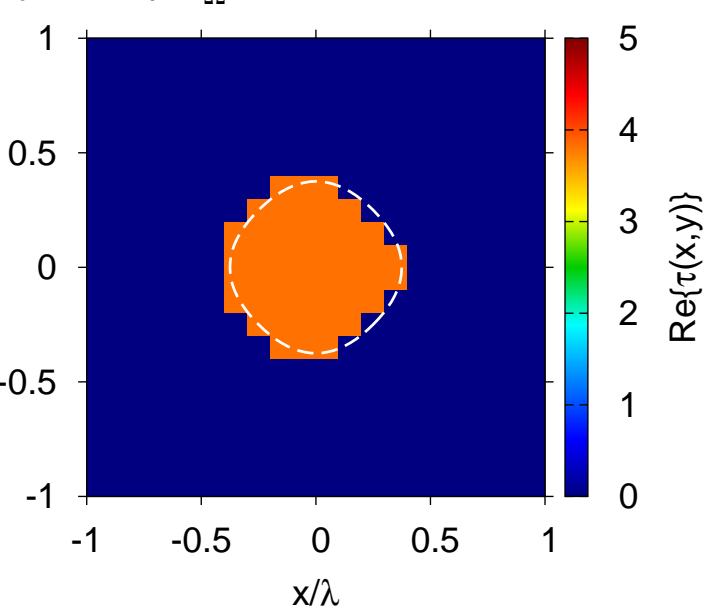

(b)
Spline Obj., $\tau_{\Omega}=4$ - DO - Noiseless Data

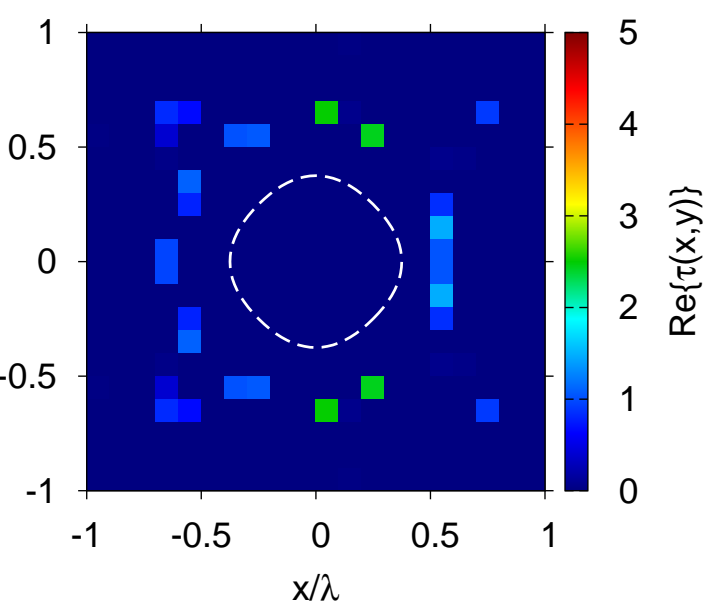

(d)

Fig. 4 - M. Salucci et al., "Learned Global Optimization ..." 


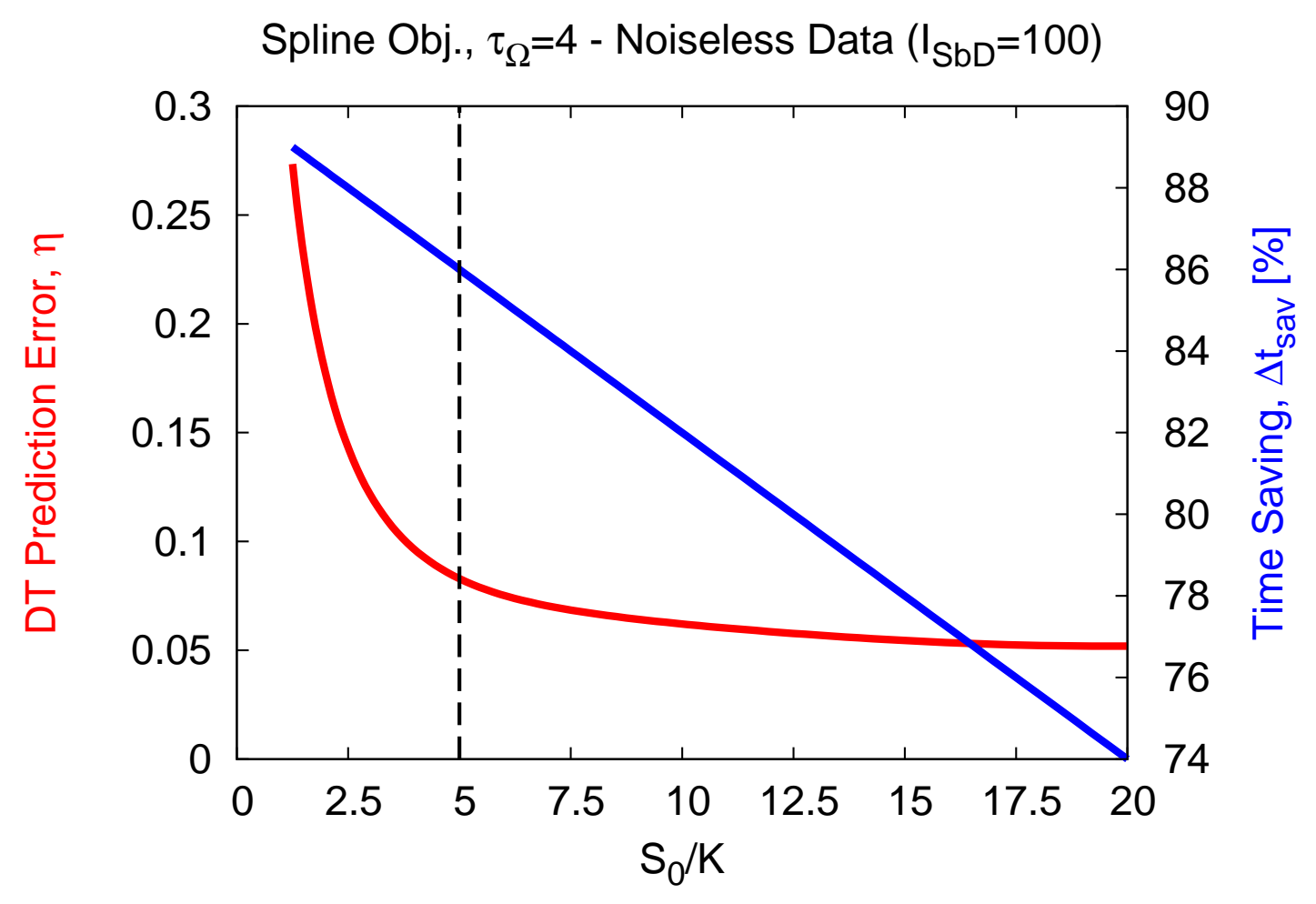

Fig. 5 - M. Salucci et al., “Learned Global Optimization ...” 


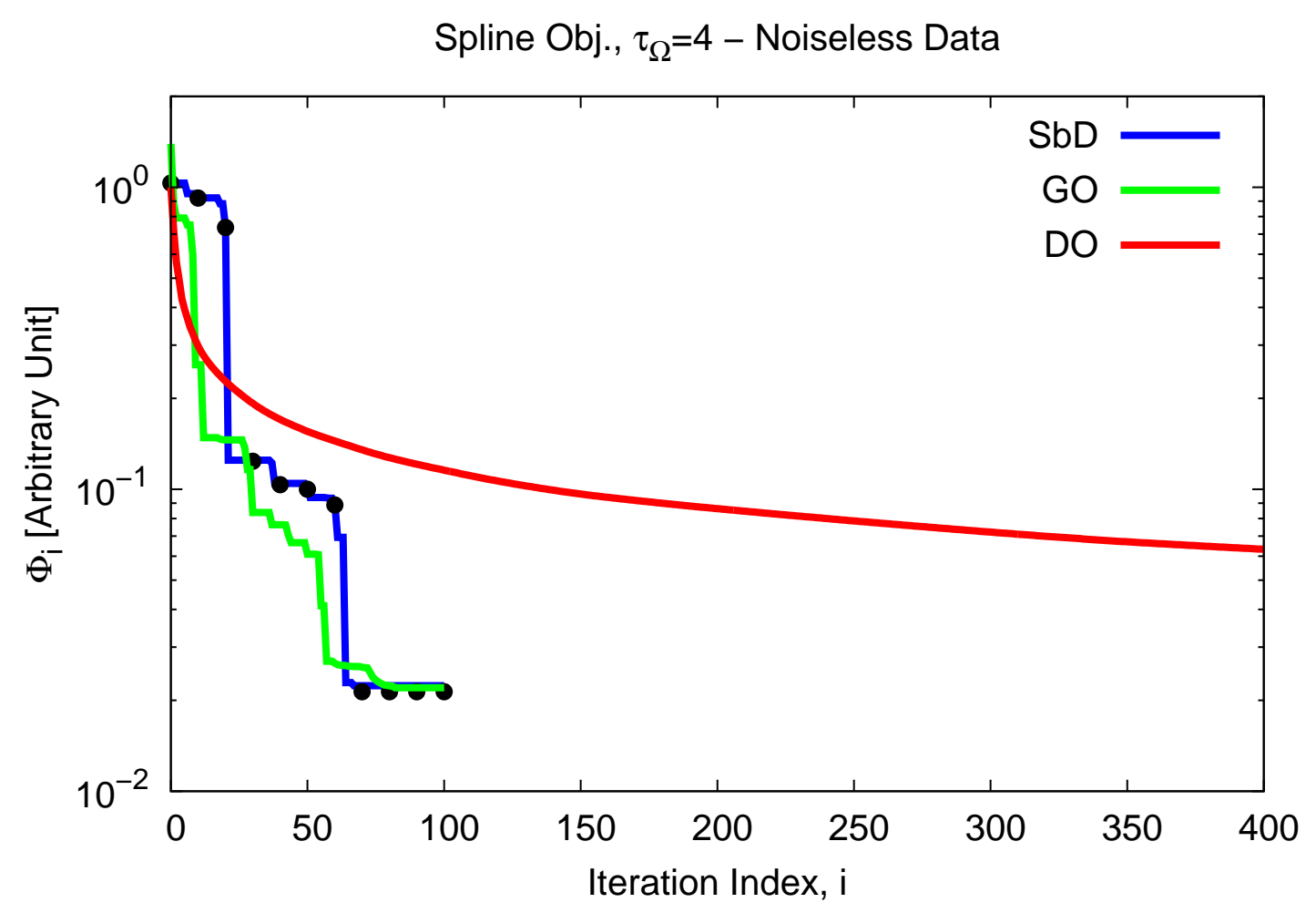

Fig. 6 - M. Salucci et al., "Learned Global Optimization ..." 


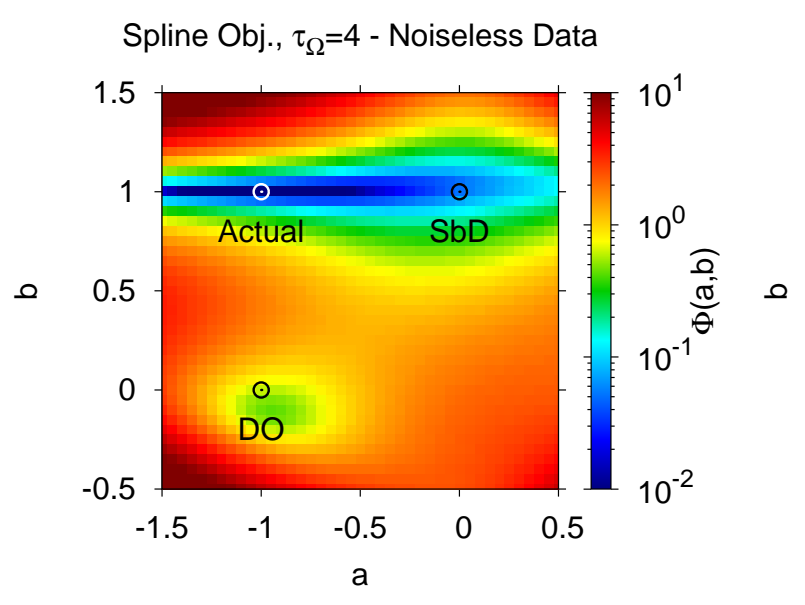

(a)

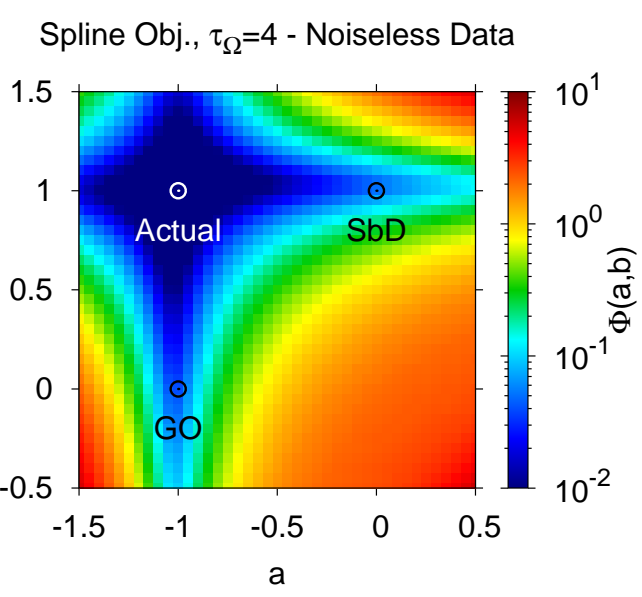

(b)

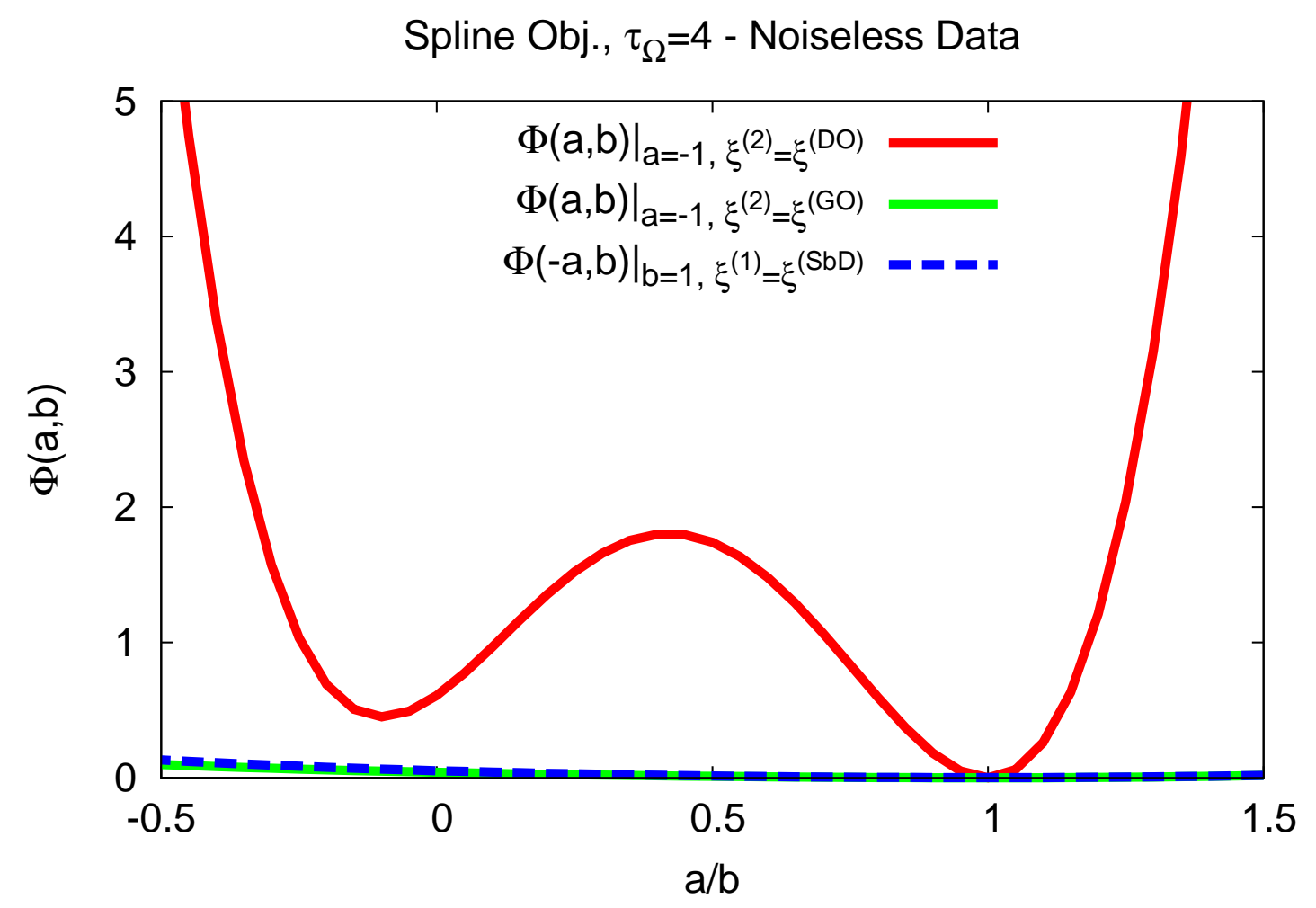

(c)

Fig. 7 - M. Salucci et al., "Learned Global Optimization ...” 


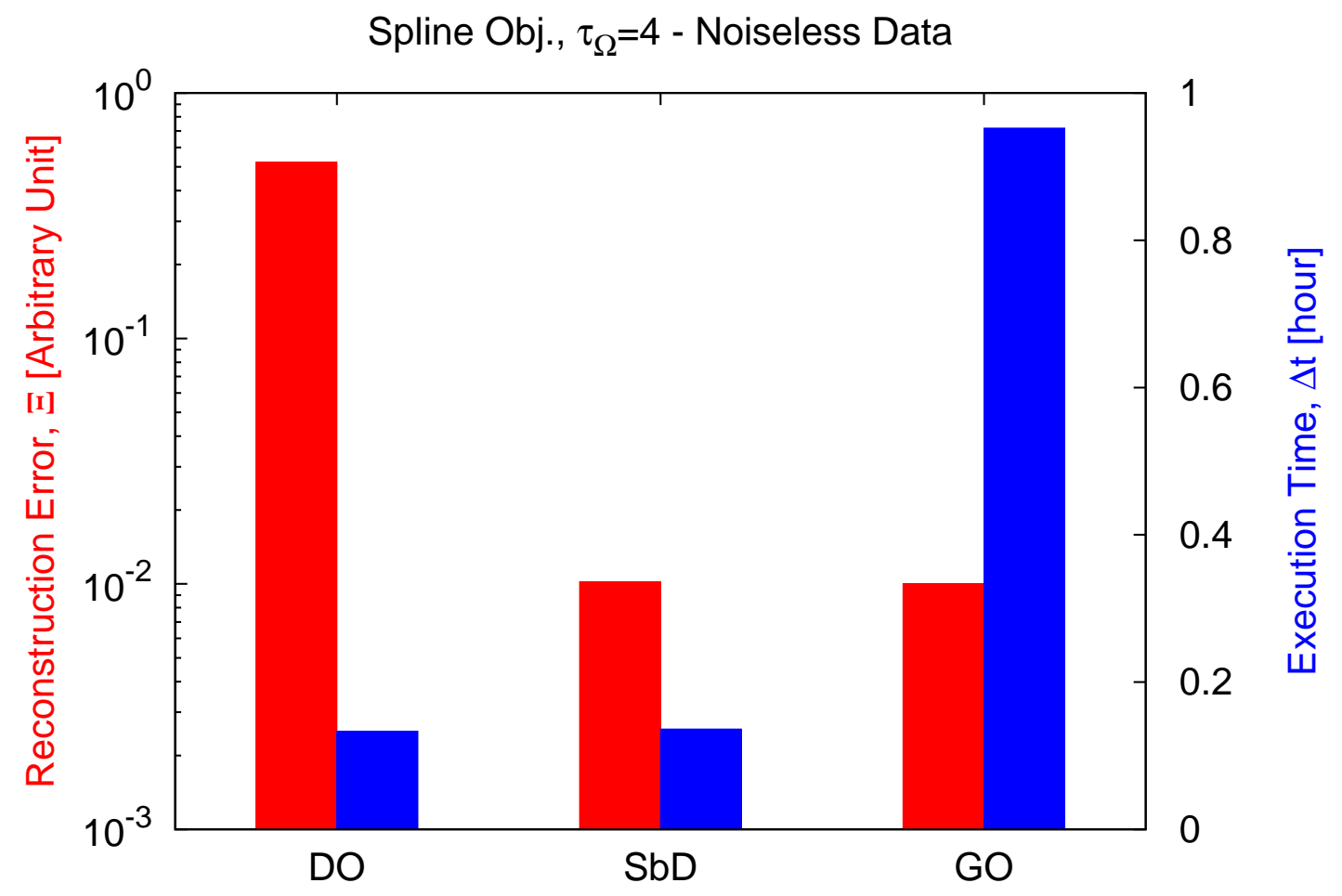

Fig. 8 - M. Salucci et al., “Learned Global Optimization ...” 

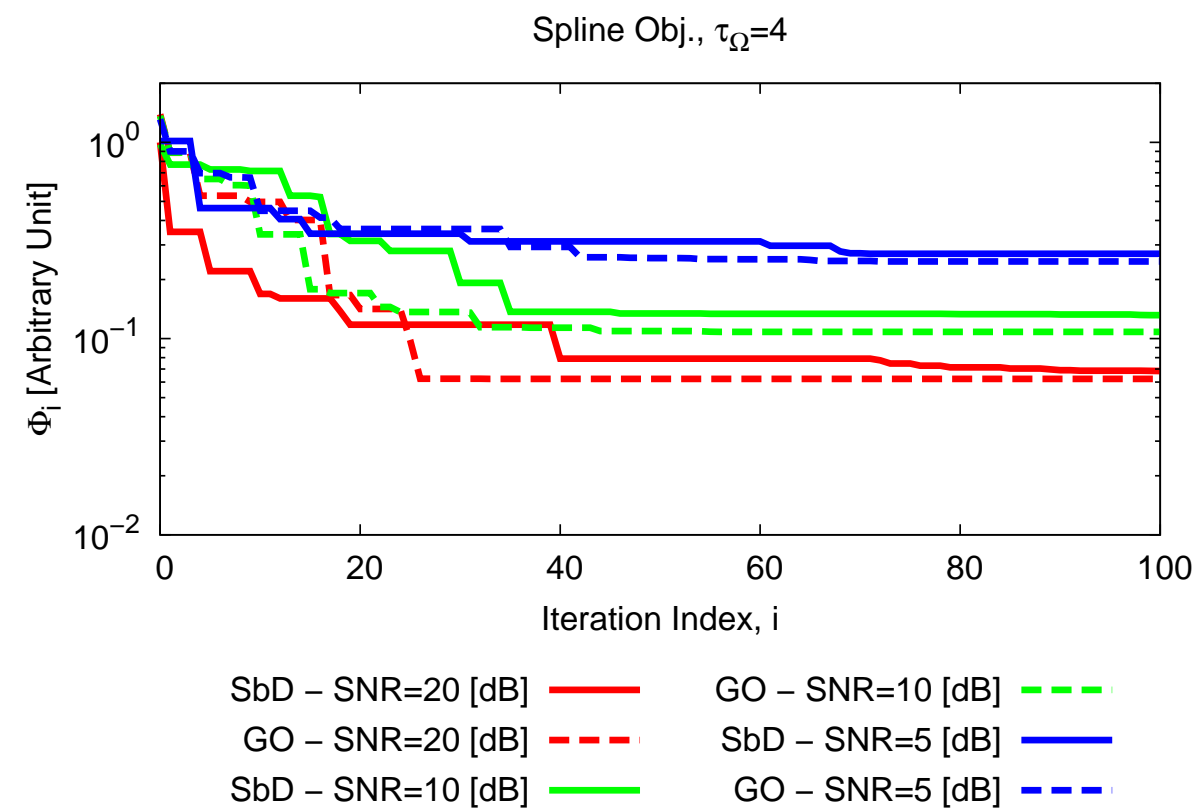

(a)

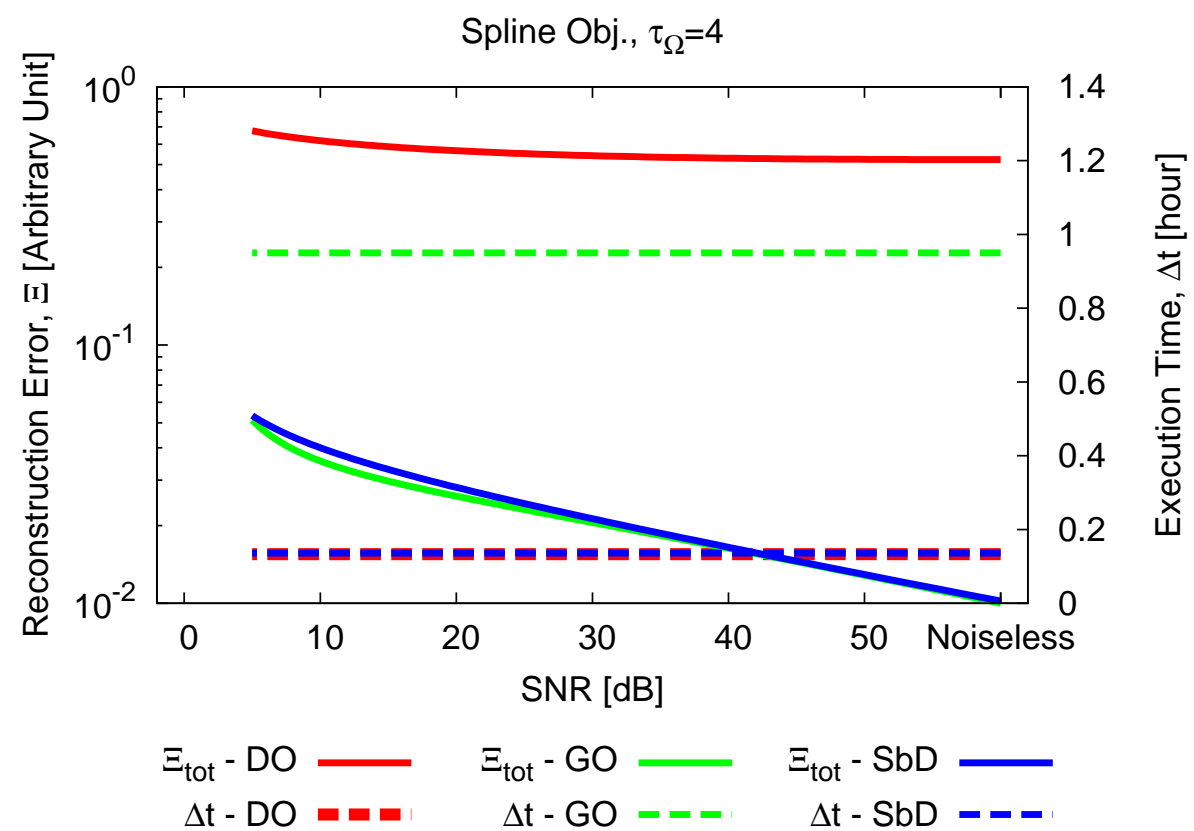

(b)

Fig. 9 - M. Salucci et al., "Learned Global Optimization ..." 


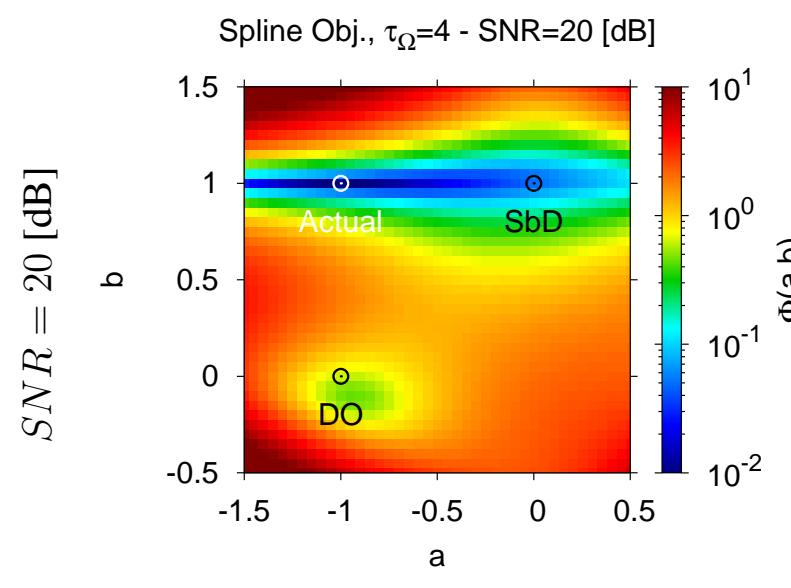

(a)

Spline Obj., $\tau_{\Omega}=4$ - SNR $=10[\mathrm{~dB}]$

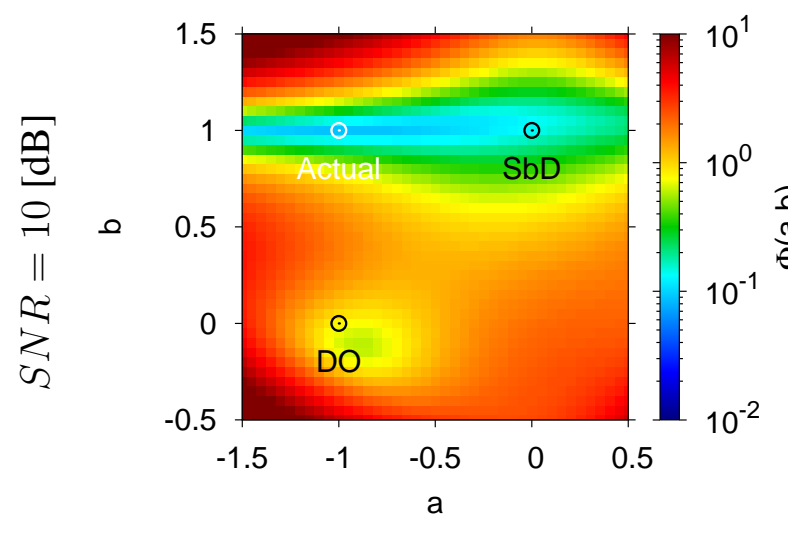

(c)

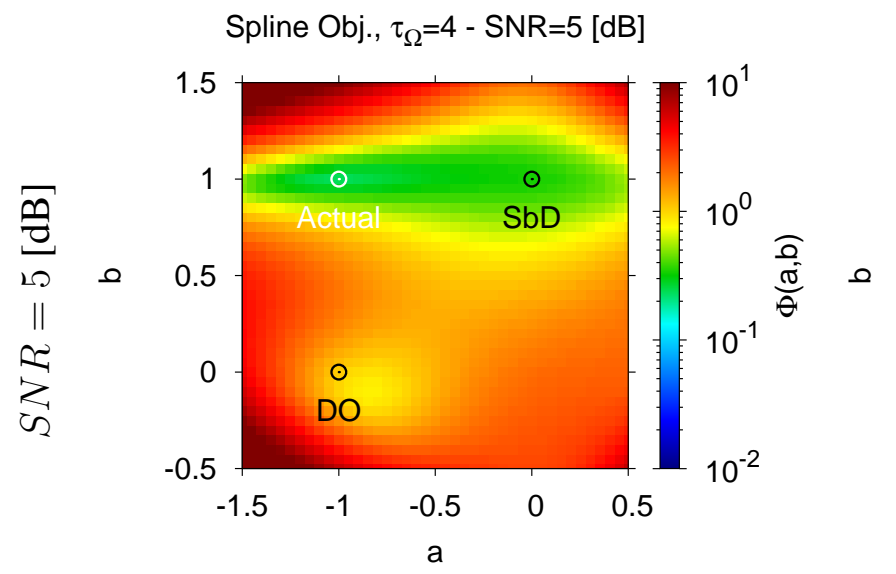

(e)

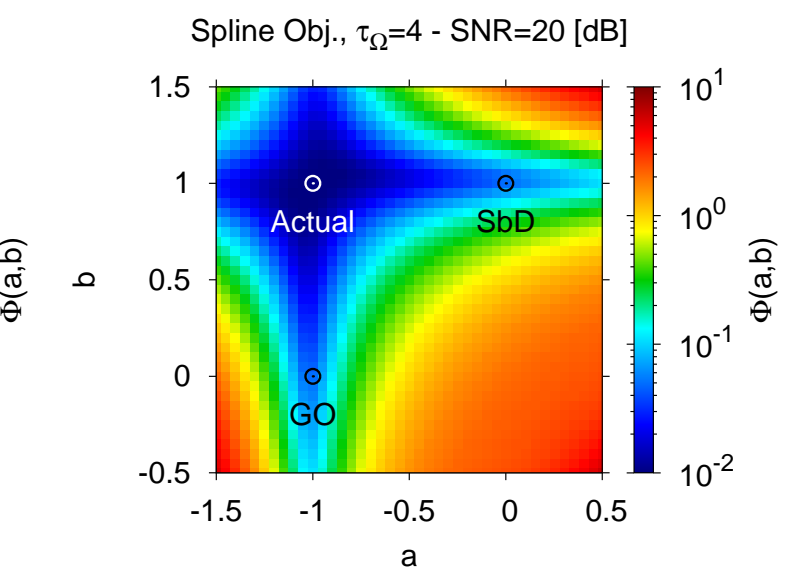

(b)

Spline Obj., $\tau_{\Omega}=4$ - SNR $=10[\mathrm{~dB}]$

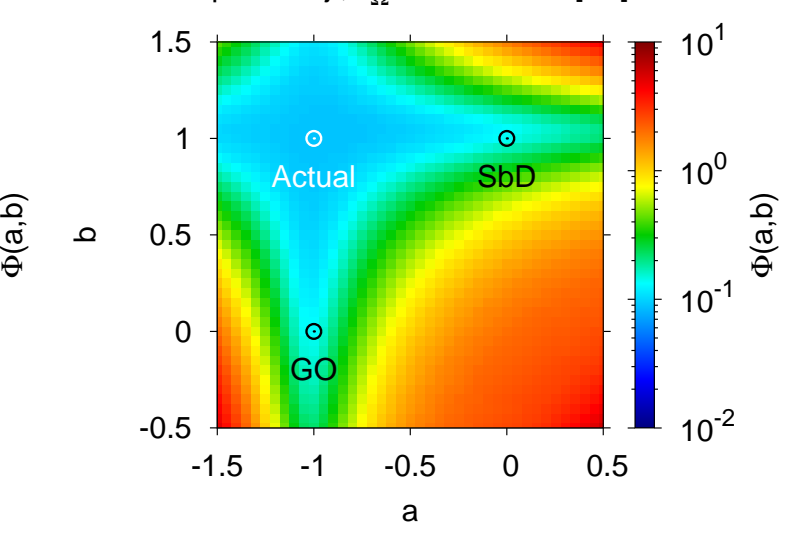

(d)

Spline Obj., $\tau_{\Omega}=4$ - SNR $=5[\mathrm{~dB}]$

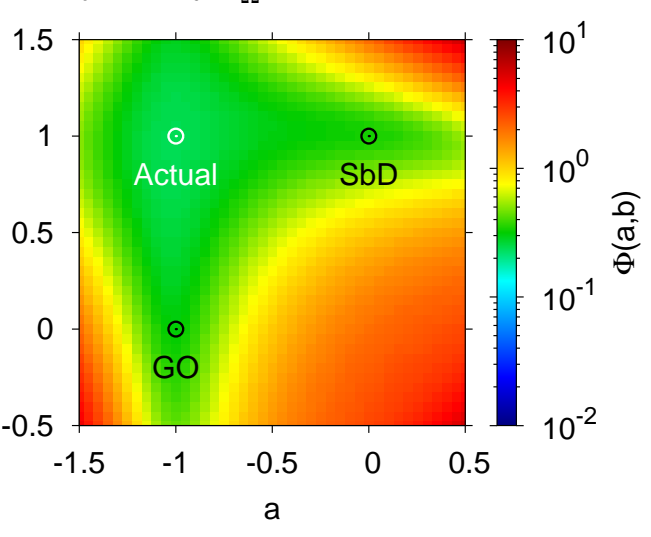

(f)

Fig. 10 - M. Salucci et al., "Learned Global Optimization ..." 


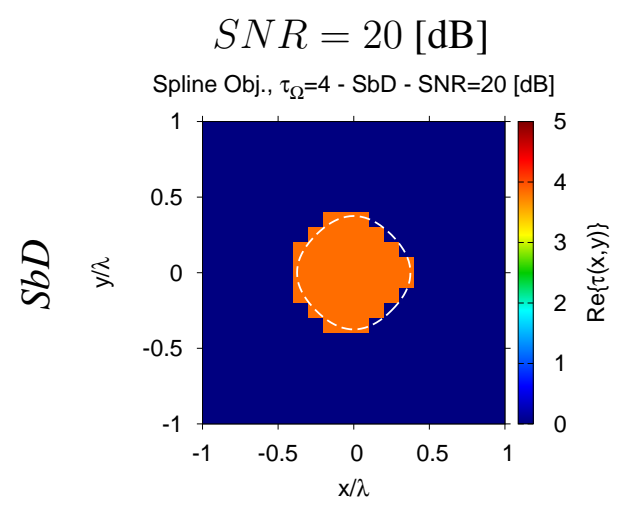

(a)

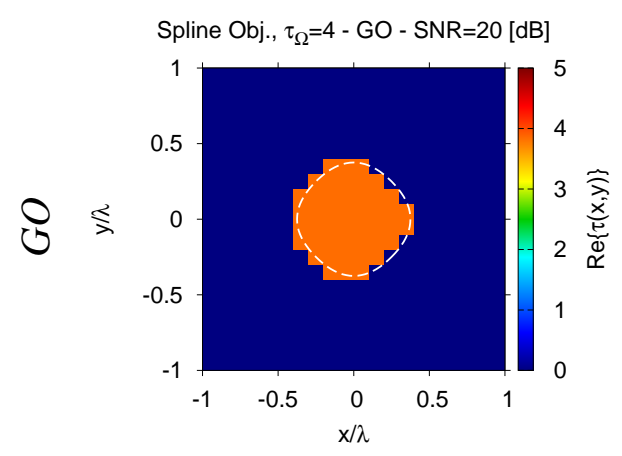

(d)

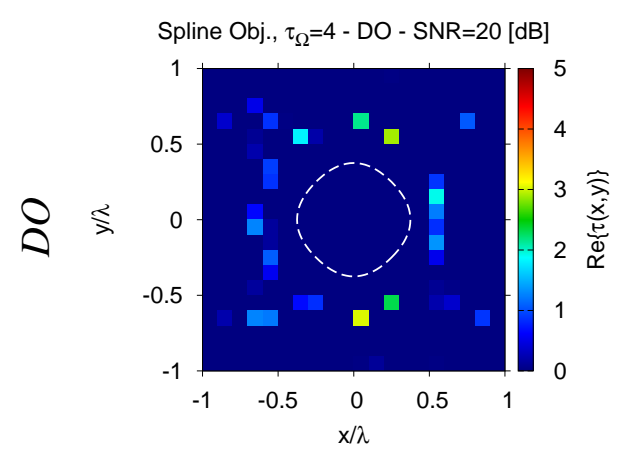

$(g)$

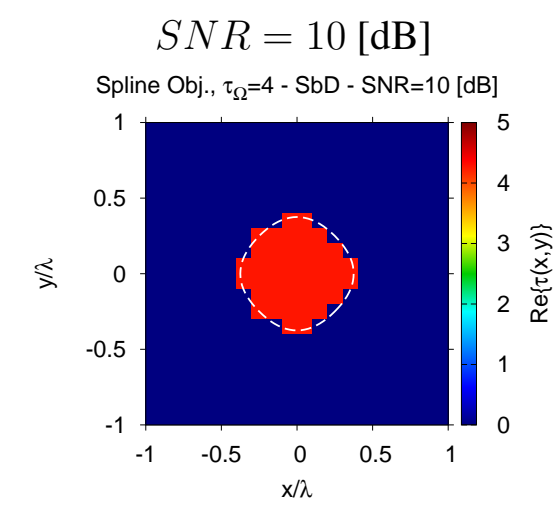

(b)

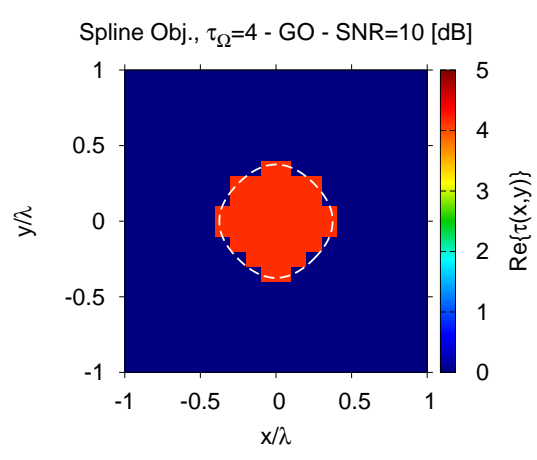

(e)

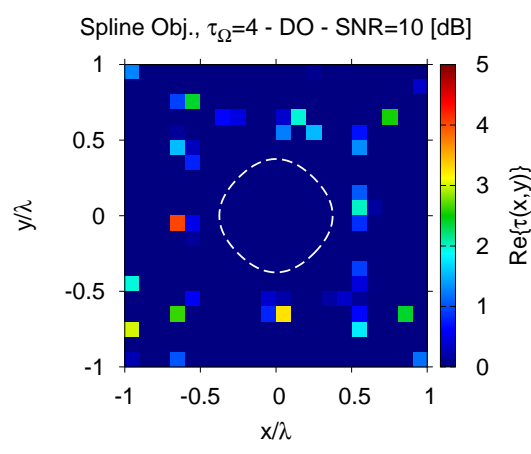

$(h)$
$S N R=5[\mathrm{~dB}]$

Spline Obj., $\tau_{\Omega}=4$ - SbD - SNR=5 [dB]

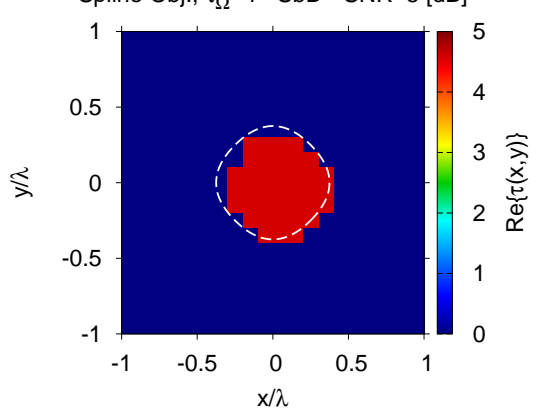

(c)

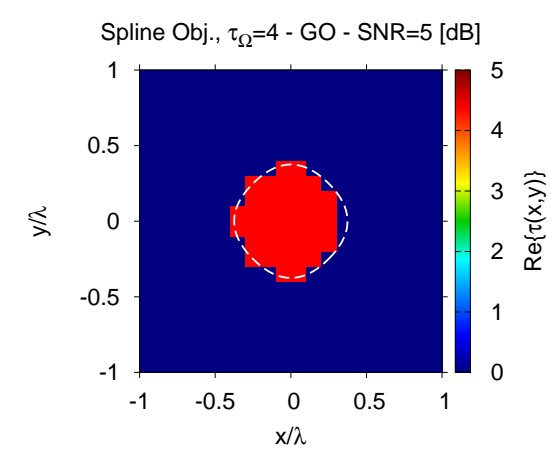

$(f)$

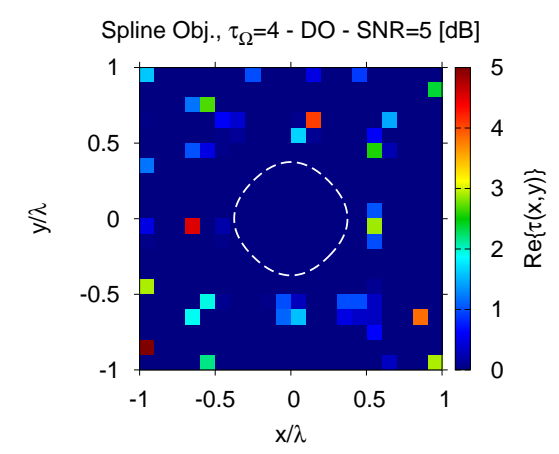

(i)

Fig. 11 - M. Salucci et al., "Learned Global Optimization ..." 


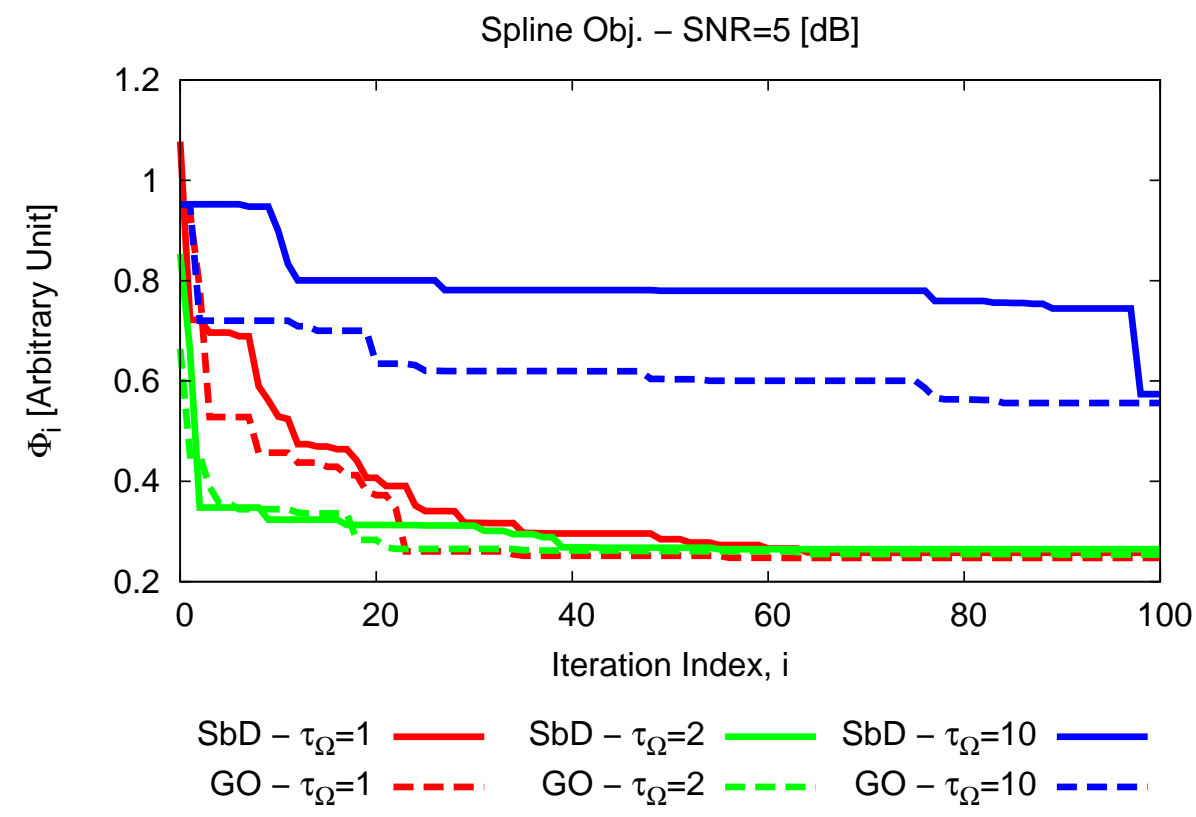

(a)

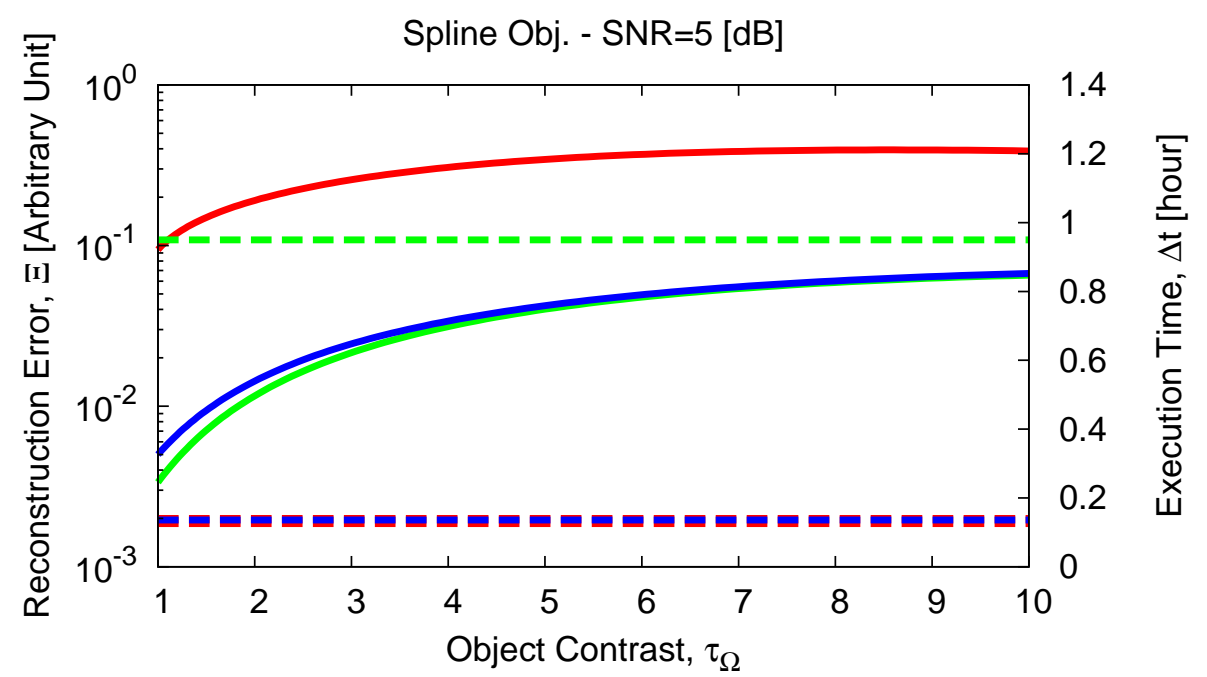

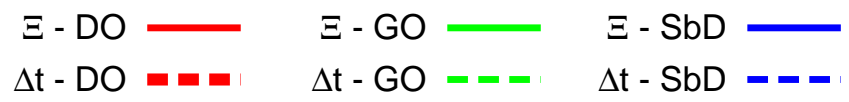

(b)

Fig. 12- M. Salucci et al., "Learned Global Optimization ..." 


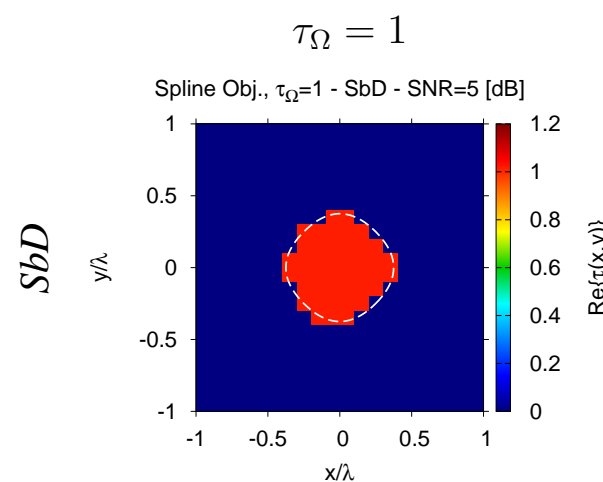

(a)

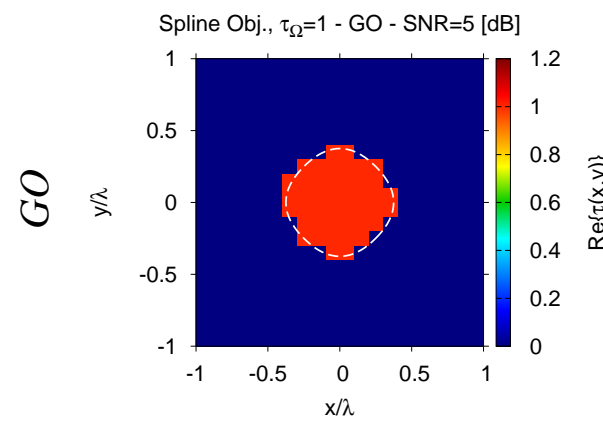

(d)

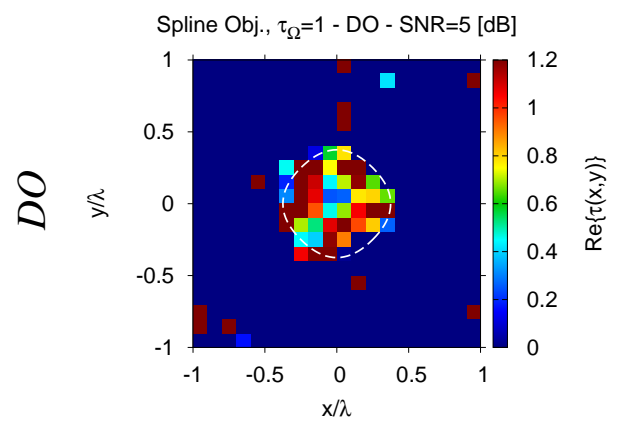

$(g)$

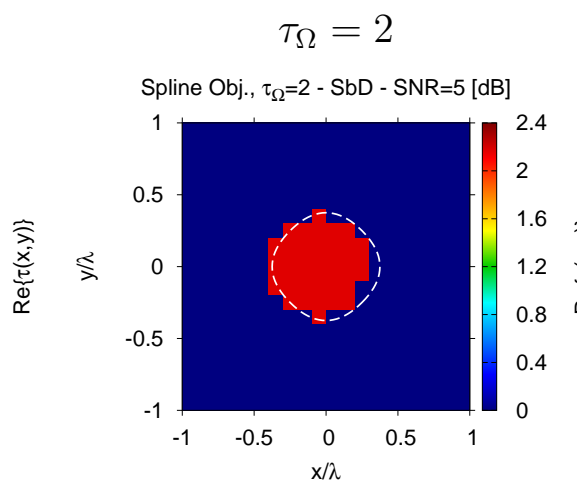

(b)

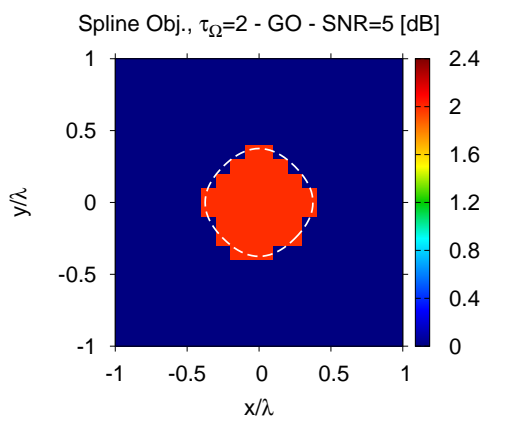

$(e)$

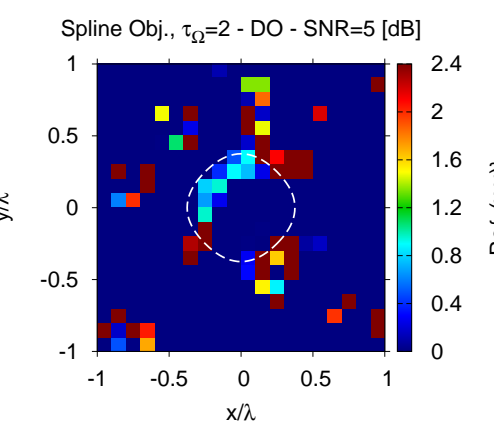

$(h)$

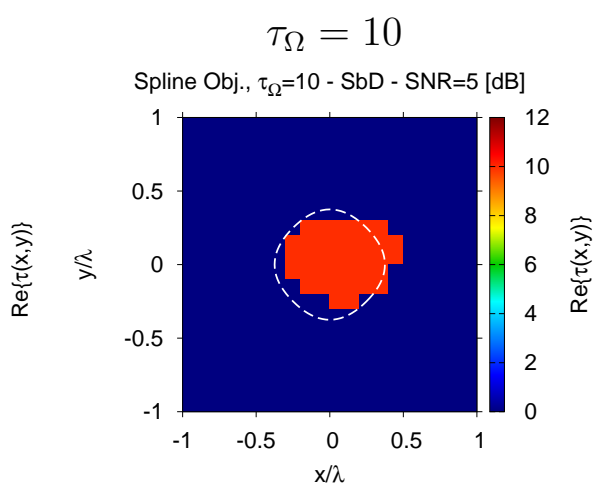

(c)

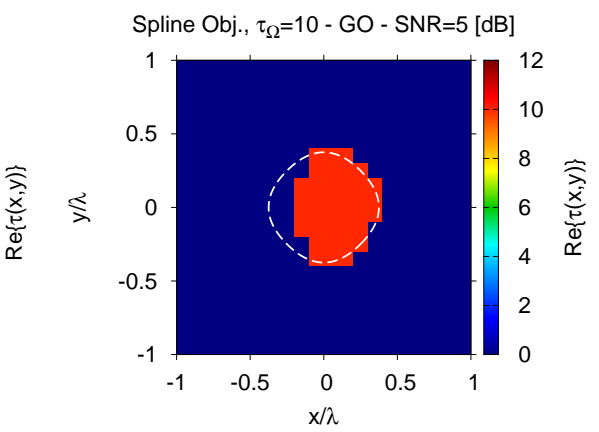

(f)

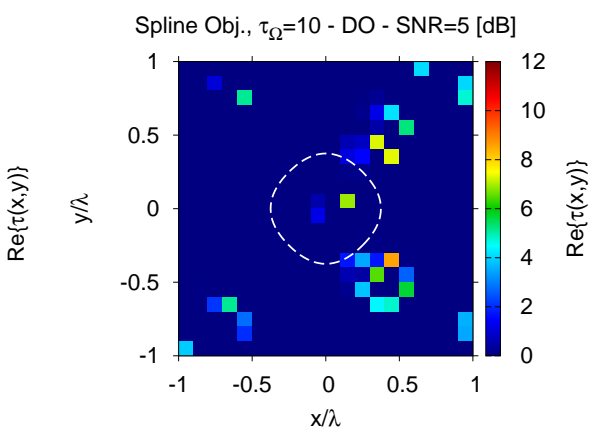

(i)

Fig. 13 - M. Salucci et al., "Learned Global Optimization ..." 


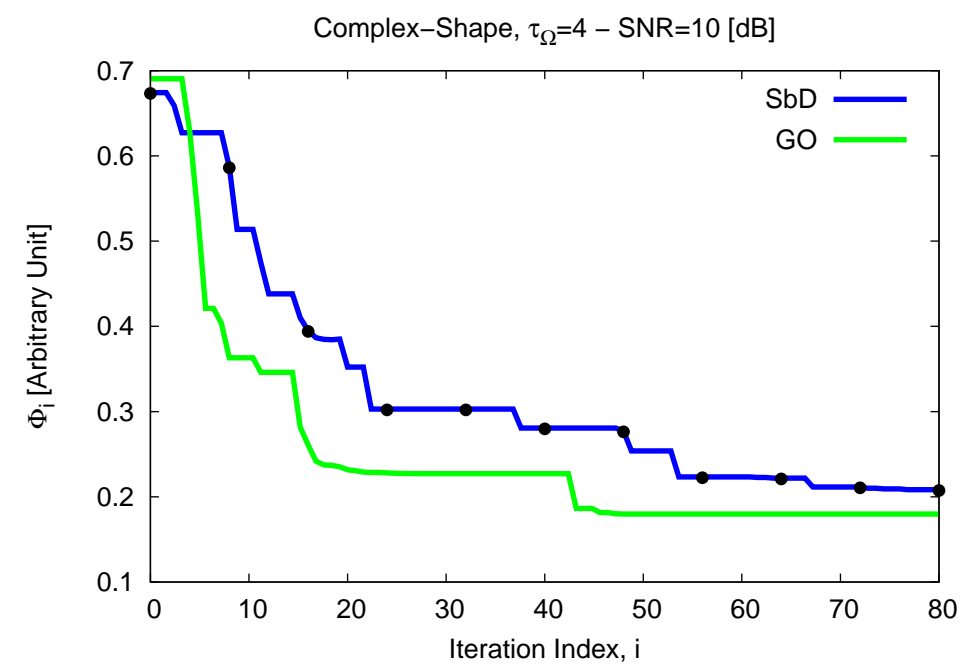

(a)

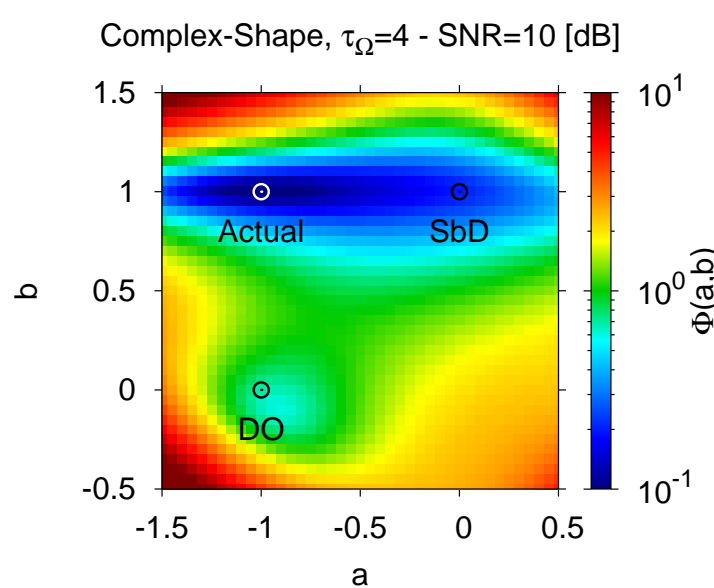

(b)
Complex-Shape, $\tau_{\Omega}=4$ - SNR $=10[\mathrm{~dB}]$

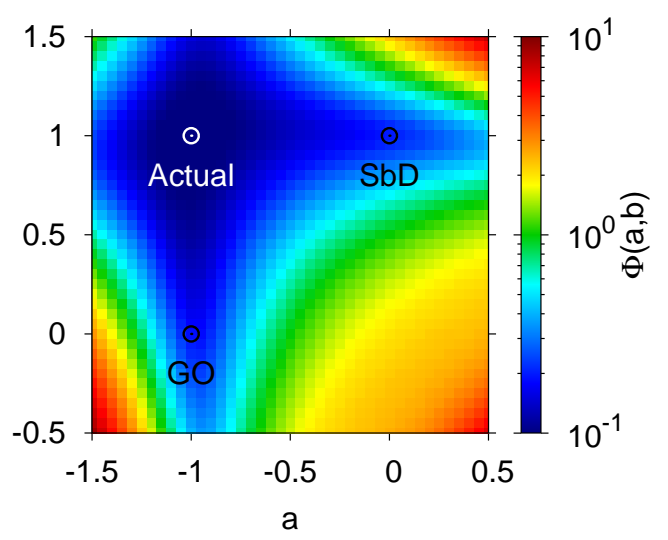

(c)

Fig. 14 - M. Salucci et al., "Learned Global Optimization ..." 


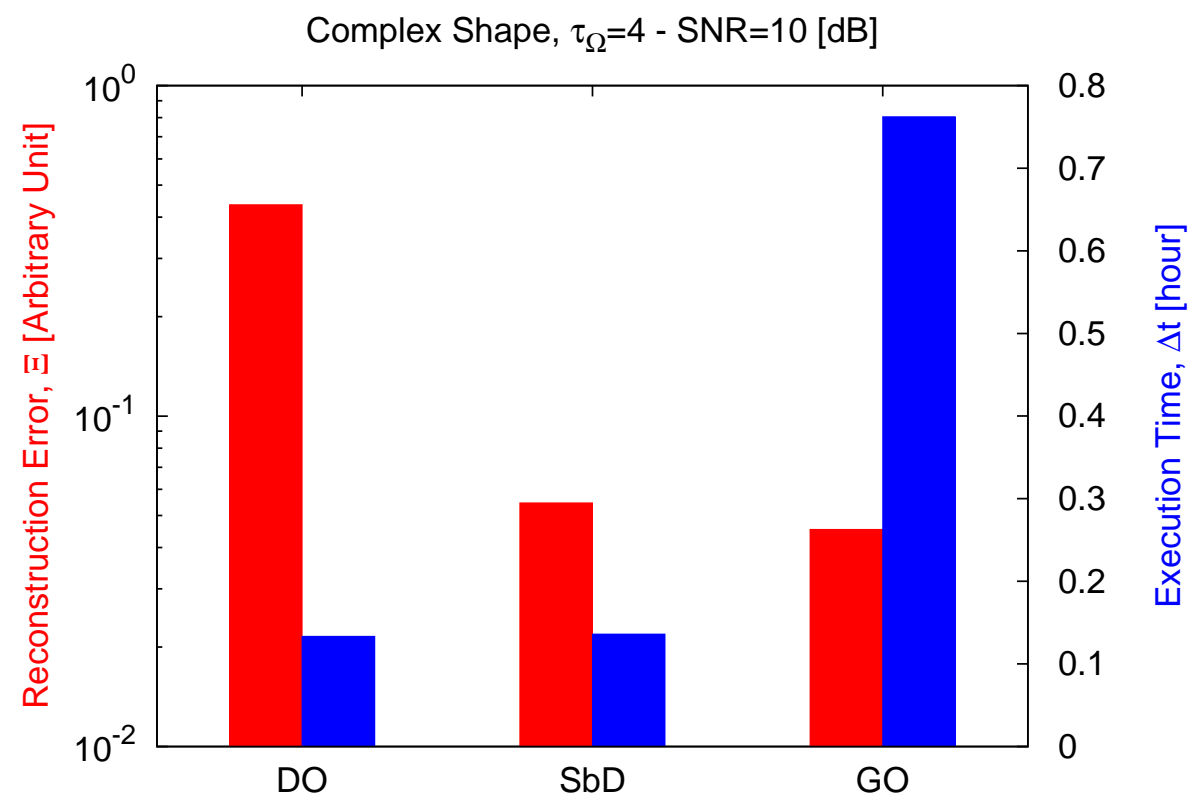

Fig. 15 - M. Salucci et al., "Learned Global Optimization ..." 


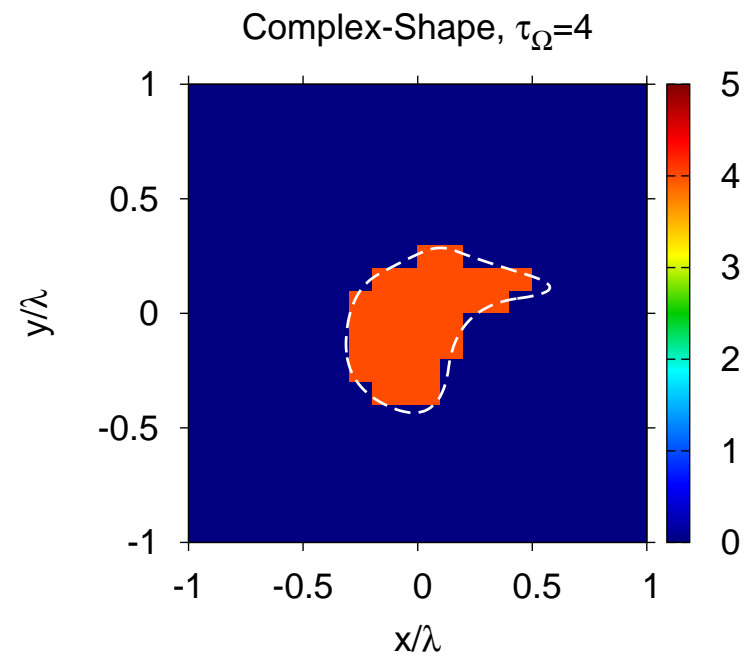

(a)

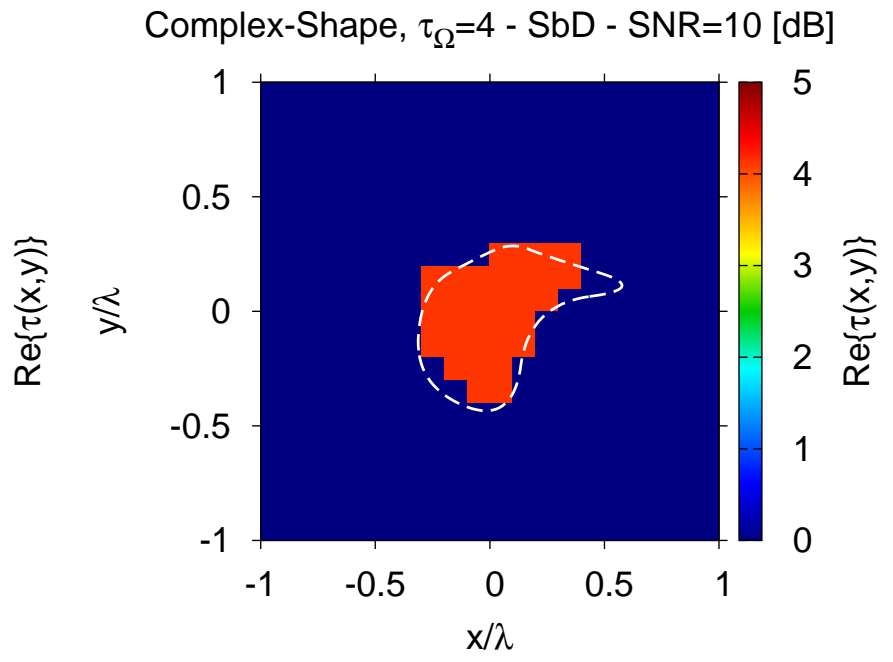

(b)

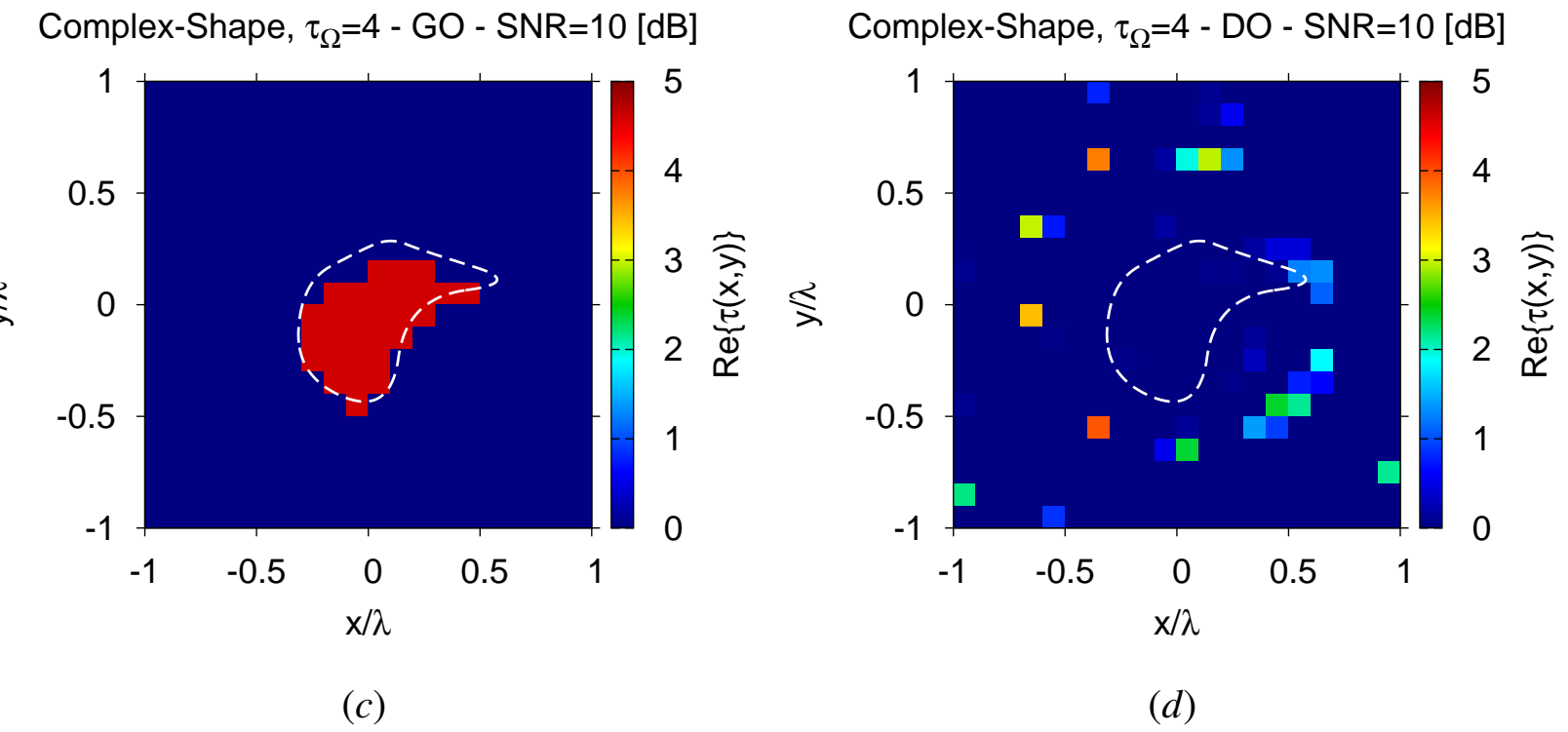

Fig. 16 - M. Salucci et al., "Learned Global Optimization ..." 


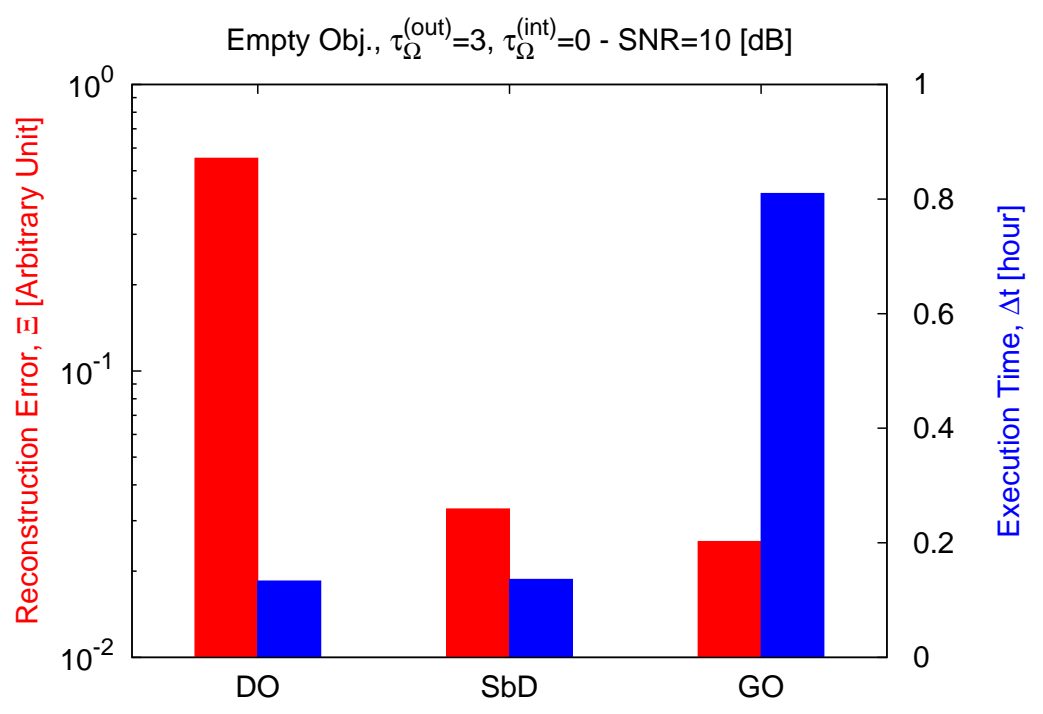

(a)

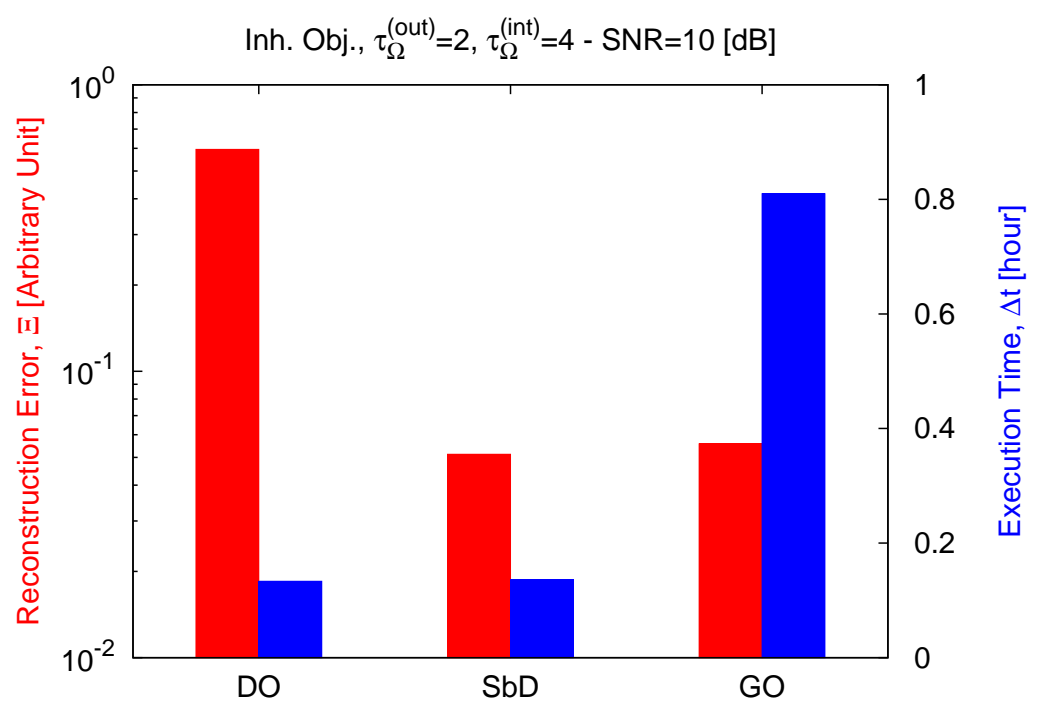

(b)

Fig. 17 - M. Salucci et al., "Learned Global Optimization ..." 


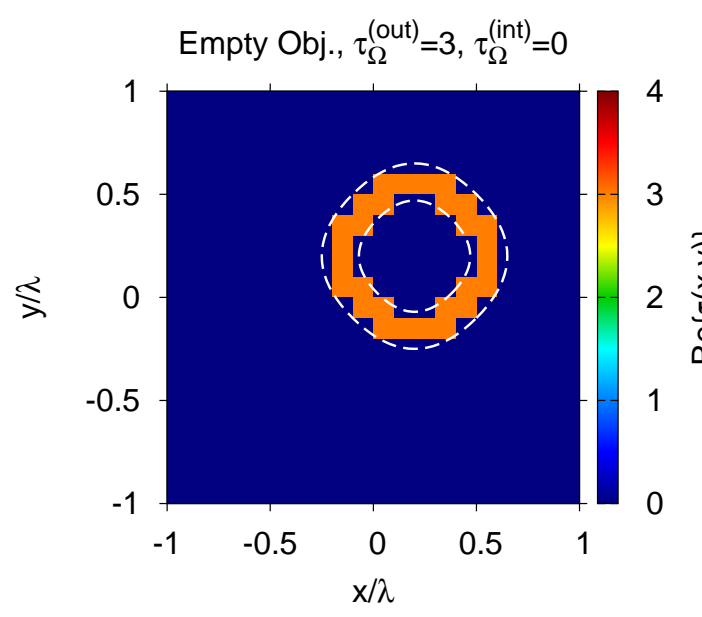

(a)

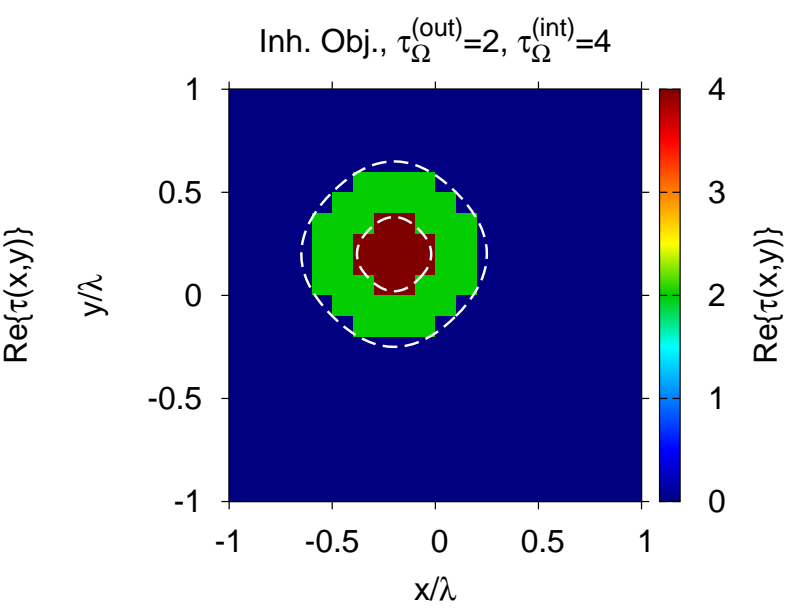

(b)

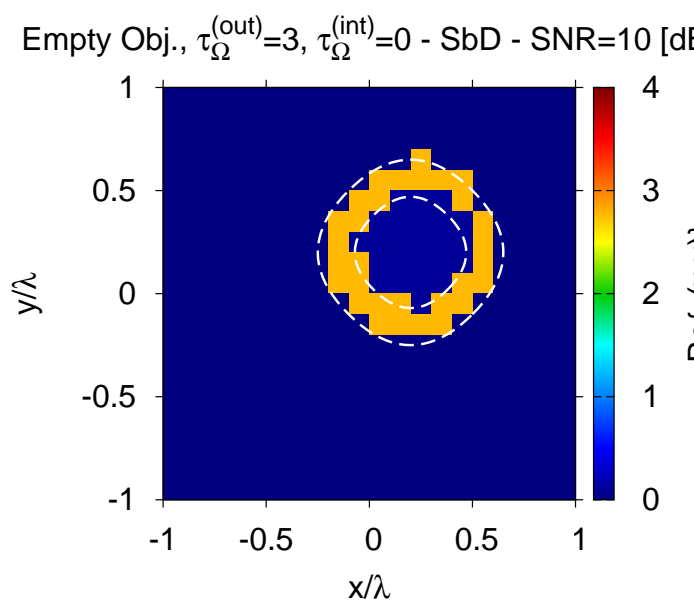

(c)
Inh. Obj., $\tau_{\Omega}^{\text {(out) }}=2, \tau_{\Omega}^{\text {(int) }}=4$ - SbD - SNR $=10[\mathrm{~dB}]$

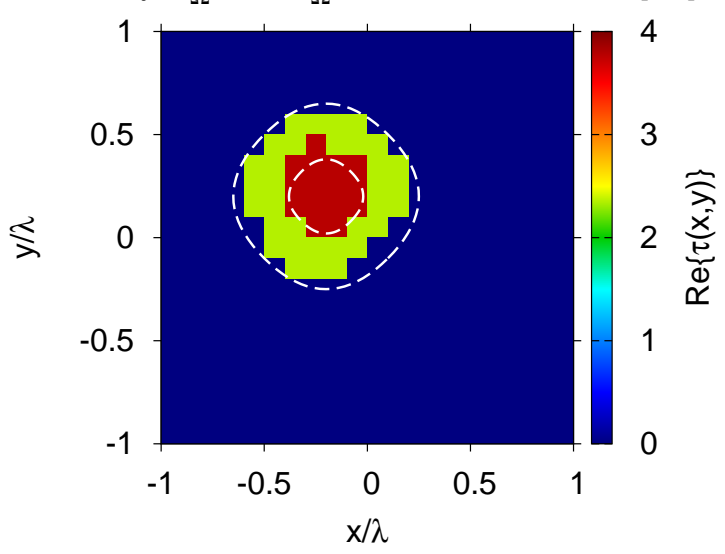

$(d)$

Fig. 18 - M. Salucci et al., "Learned Global Optimization ..." 


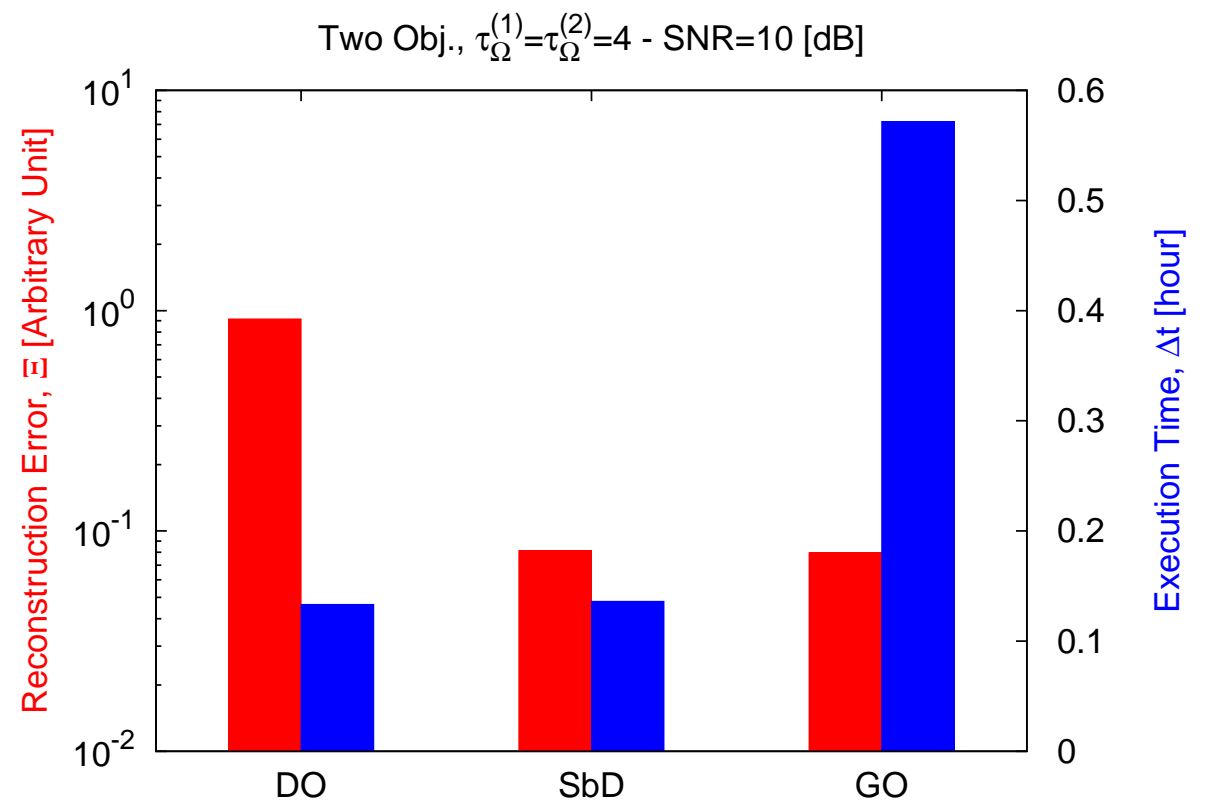

Fig. 19 - M. Salucci et al., "Learned Global Optimization ..." 


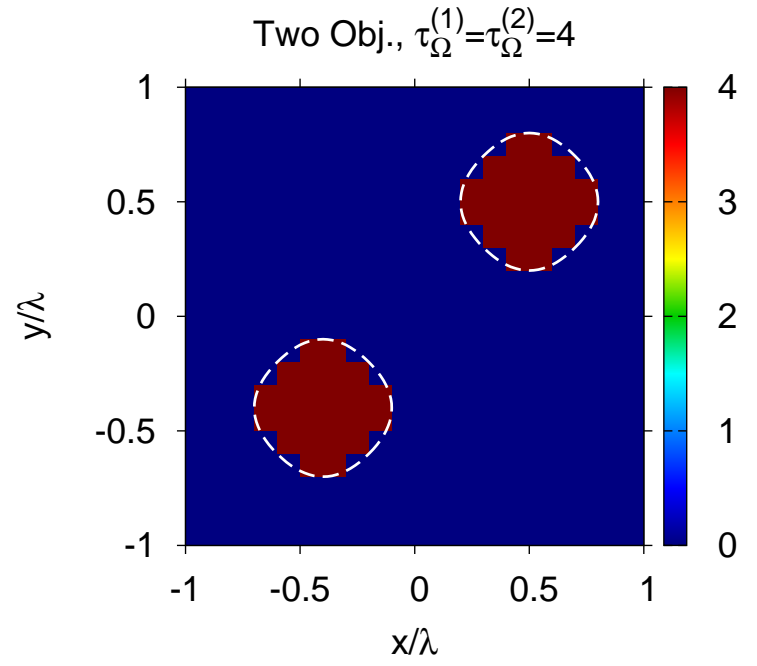

(a)

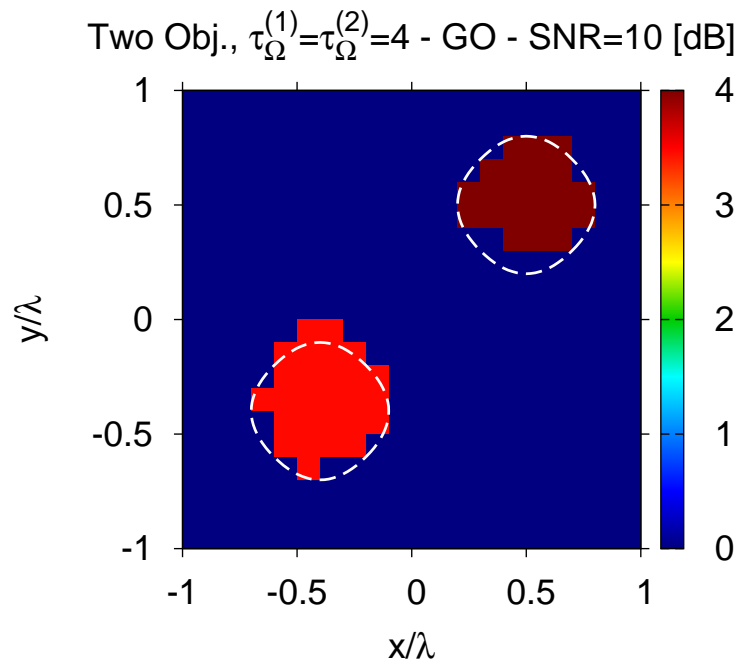

(c)

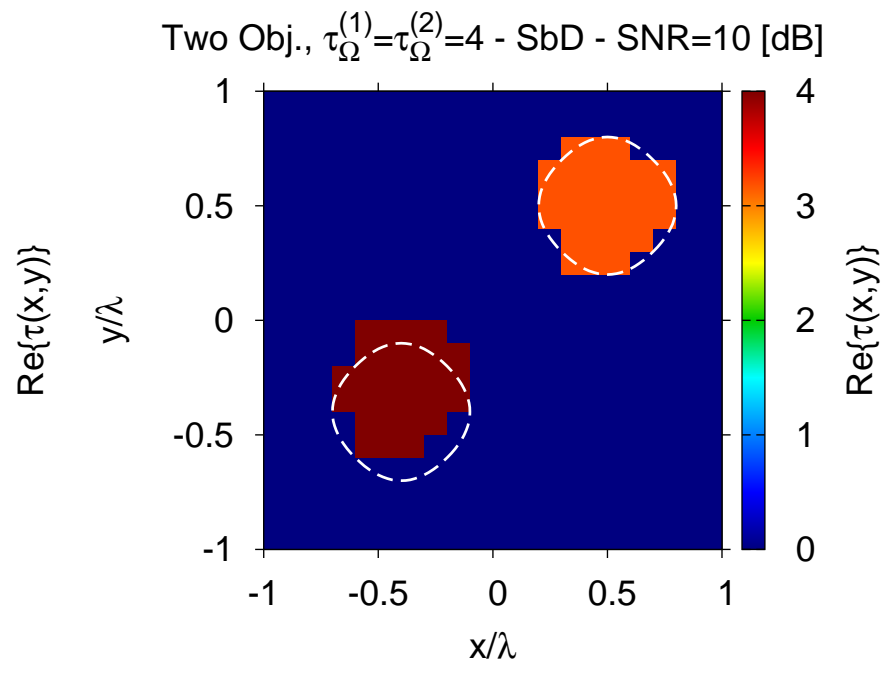

(b)

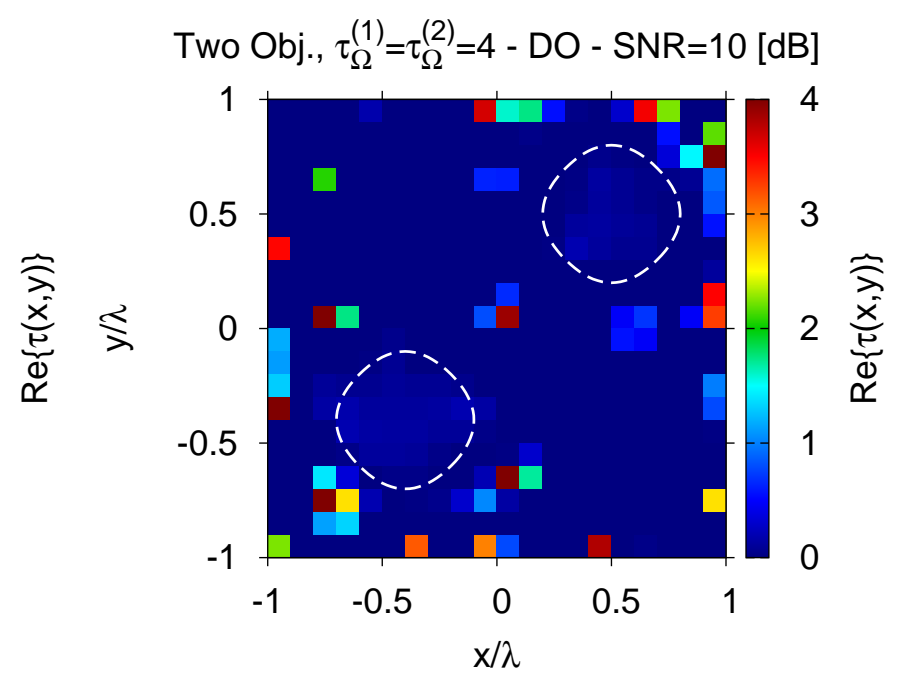

(d)

Fig. 20 - M. Salucci et al., "Learned Global Optimization ..." 


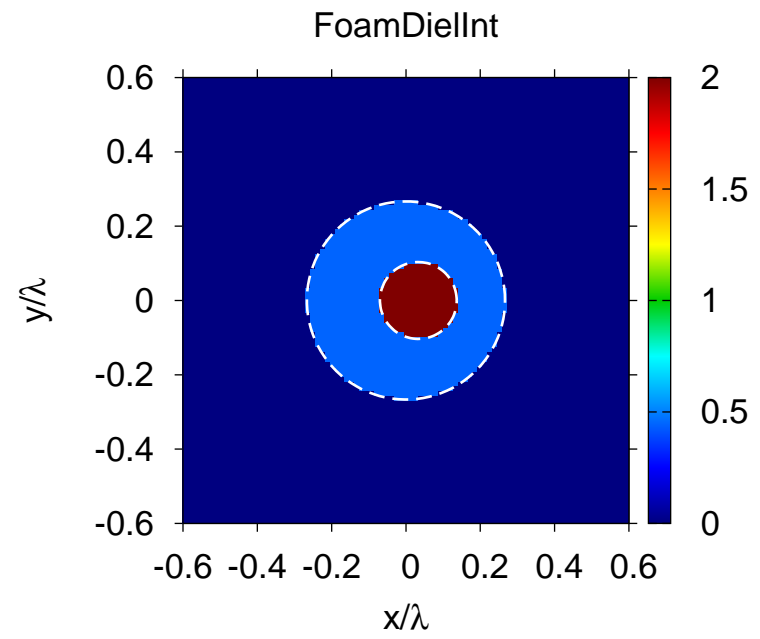

(a)

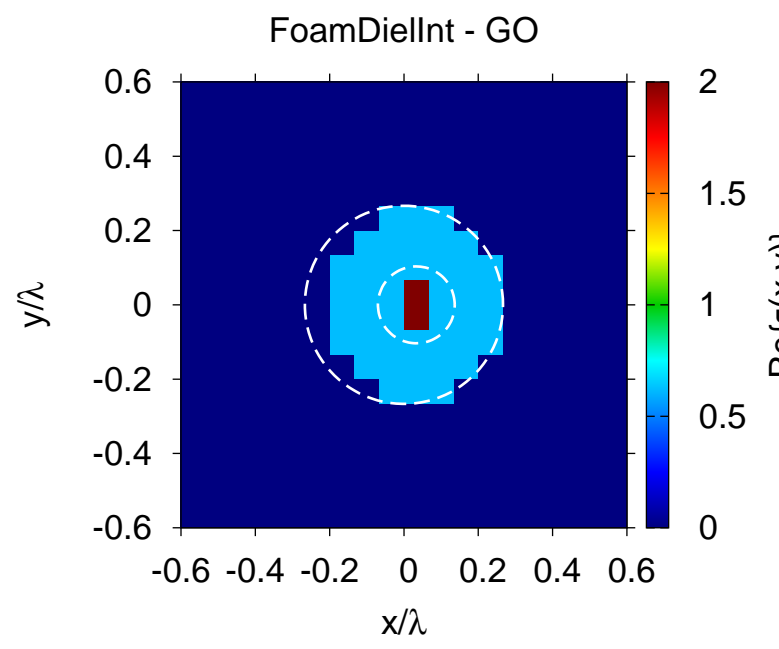

(c)

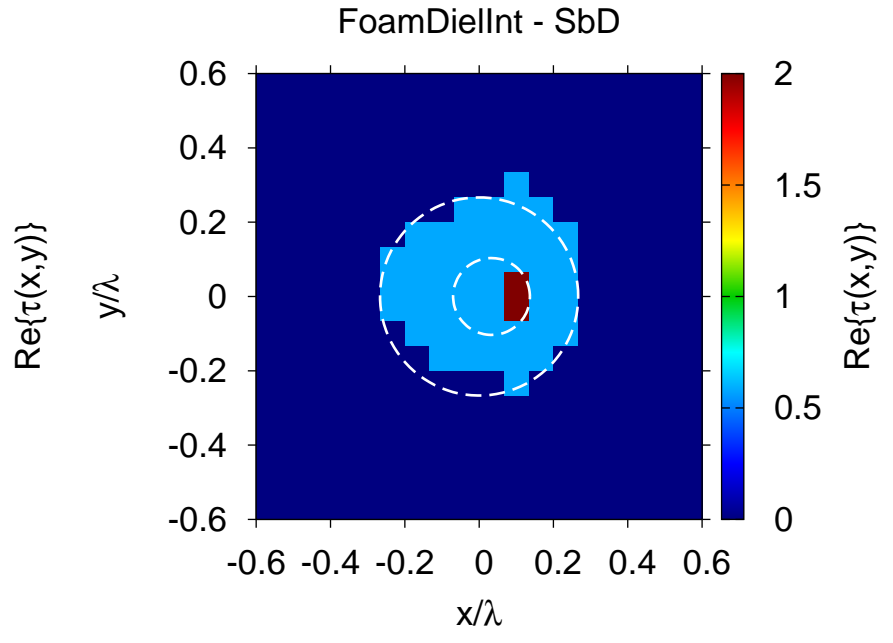

(b)

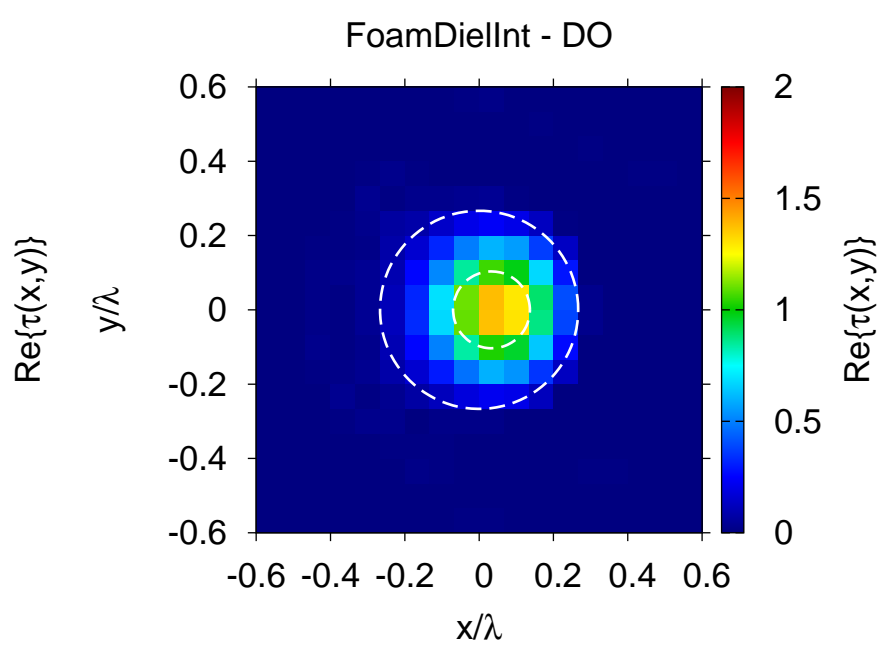

$(d)$

Fig. 21 - M. Salucci et al., "Learned Global Optimization ..." 


\begin{tabular}{|c|c|c|c|c|c|c|c|}
\hline Object & $K$ & $S_{0}$ & $I_{S b D}$ & Profile & $\left(x_{\Omega}, y_{\Omega}\right)[\lambda]$ & $Q$ & $\underline{\rho}[\lambda]$ \\
\hline \hline Fig. 4(a) & 8 & 40 & 100 & $\partial \Omega$ & $(0,0)$ & 4 & $\{0.5,0.5,0.5,0.5\}$ \\
\hline Fig. 16(a) & 12 & 60 & 80 & $\partial \Omega$ & $(0.1,0.1)$ & 8 & $\{0.6,0.2,0.2,0.2,0.4,0.6,0.6,0.1\}$ \\
\hline Fig. 18(a) & 11 & 55 & 85 & $\partial \Omega^{(\text {out })}$ & $(0.2,0.2)$ & 4 & $\{0.6,0.6,0.6,0.6\}$ \\
& & & & $\partial \Omega^{(\text {int })}$ & $(0.2,0.2)$ & 4 & $\{0.36,0.36,0.36,0.36\}$ \\
\hline Fig. 18(b) & 11 & 55 & 85 & $\partial \Omega^{(\text {out })}$ & $(-0.2,0.2)$ & 4 & $\{0.6,0.6,0.6,0.6\}$ \\
& & & & $\partial \Omega^{(\text {int })}$ & $(-0.2,0.2)$ & 4 & $\{0.24,0.24,0.24,0.24\}$ \\
\hline Fig. 20(a) & 16 & 80 & 60 & $\partial \Omega^{(1)}$ & $(-0.4,-0.4)$ & 4 & $\{0.4,0.4,0.4,0.4\}$ \\
& & & & $\partial \Omega^{(2)}$ & $(0.5,0.5)$ & 4 & $\{0.4,0.4,0.4,0.4\}$ \\
\hline
\end{tabular}

Tab. I - M. Salucci et al., "Learned Global Optimization ..." 